\title{
On the First Initial-Boundary Value Problem of Compressible Viscous Fluid Motion
}

\author{
By
}

Atusi TANI*

Up to the present day many kinds of mathematical discussions on incompressible viscous fluid motion have fully developed (cf. [32, 36]). As for compressible viscous one, however, there have been only a few works on it. In 1959 Serrin [50] proved the uniqueness theorem in a bounded domain, making use of the classical energy method. In 1962 Nash [44] tried to show the existence theorem in $R^{3}$, but it seems to the author that he has failed. Independently of them Itaya succeeded to prove the existence and the uniqueness theorems on the Cauchy problem for it in [24-28], using Tikhonov's fixed point theorem.

Now in the present paper, we shall show that the first initial-boundary value problem for it can uniquely be solved under suitable assumptions for the initial-boundary data and for the boundary of the domain, from the classical point of view.

In $\S 1$ an exact statement and the main theorem (Theorem 1) will be found. In $\S 2$ we perform the characteristic transformation and mention the theorem of the transformed problem (Theorem 2). Firstly we prove Theorem 2 and then show that Theorem 2 implies Theorem 1 in the last section $\S 8$. In $\S \S 3-5$ linear equations connected with the transformed equations are treated. In more detail, in $\S 3.1$ we briefly state some basic results for a fundamental solution in the whole space $R^{3}$ due to Eidel'man [9, 18] and Pogorzelski [46-48] (cf. [25]). In $§ 3.2$ we check the basic condition of uniform solvability due to Solomjak [52, 54], which is essential for the study of the boundary value problem in applied mathematics, corresponding to the Lopatinsky condition for the

Communicated by K. Itô, September 25, 1976.

* Department of Applied Physics, Tokyo Institute of Technology, Tokyo 152, Japan.

Present Address: Department of Mathematics, Faculty of Engineering, Keio University, 832, Hiyoshi, Kohoku, Yokohama 223, Japan. 
elliptic system in the sense of Petrowsky [37-39] and the complementing condition for the elliptic system in the Douglis-Nirenberg sense [1, $8,53]$. Once it is shown that this condition holds, we can construct the Poisson kernel and the Green matrix $[1,10-12,14,15,22,29-31,54]$. We estimate the Green matrix in $\S 4$ and the solution in $\S 5$. Making use of these results we give a proof of the existence in $\S 6$ by the method of successive approximation and that of the uniqueness in $\S 7$, therefore the proof of Theorem 2 is completed.

Acknowledgement. Deep gratitude is due to Professor N. Itaya for his kind advices and encouragements throughout this research.

\section{$\S 1$. Introduction}

1. 1. Statement of the Problem. Compressible viscous isotropic Newtonian fluid motion is described by five differential equations corresponding to the law of mass, momentum and energy as follows (as for kinematics, see, for example, $[34,35,40,51,55])$ :

$$
\begin{aligned}
& \frac{D \rho}{D t}=-\rho \frac{\partial v_{k}}{\partial x_{k}} \\
& \frac{D v_{i}}{D t}=\frac{1}{\rho} \frac{\partial}{\partial x_{i}}\left(\mu^{\prime} \frac{\partial v_{k}}{\partial x_{k}}\right)+\frac{1}{\rho} \frac{\partial}{\partial x_{k}}\left[\mu\left(\frac{\partial v_{i}}{\partial x_{k}}+\frac{\partial v_{k}}{\partial x_{i}}\right)\right] \\
& -\frac{1}{\rho} \frac{\partial p}{\partial x_{i}}+f_{i}(i=1,2,3), \\
& \text { (1. 3) } \frac{D S}{D t}=\frac{1}{\rho \theta} \frac{\partial}{\partial x_{k}}\left(\kappa \frac{\partial \theta}{\partial x_{k}}\right)+\frac{\mu}{2 \rho \theta}\left(\frac{\partial v_{i}}{\partial x_{k}}+\frac{\partial v_{k}}{\partial x_{i}}\right)^{2}+\frac{\mu^{\prime}}{\rho \theta}\left(\frac{\partial v_{k}}{\partial x_{k}}\right)^{2} \text {, }
\end{aligned}
$$

where $\rho$, density; $v=\left(v_{1}, v_{2}, v_{3}\right)$, velocity; $\mu$, coefficient of viscosity; $\mu^{\prime}$, second coefficient of viscosity; $\kappa$, coefficient of heat conduction; $p$, pressure; $f=\left(f_{1}, f_{2}, f_{3}\right)$, outer force; $S$, entropy; $\theta$, absolute temperature; $D / D t$ $=\partial / \partial t+v_{k} \cdot \partial / \partial x_{k}$.

The summation convention will always be used unless the contrary is stated explicitly.

By the physical structure of fluid we can assume that $\mu, \mu^{\prime}, \kappa, p$ and $S$ are functions of $\rho$ and $\theta$ such that $\mu^{\prime}+\frac{2}{3} \mu \geqq 0$ and $\mu, \kappa, p, S_{\theta}>0$.

If $S$ be smooth, then using the equation (1.1) we have 


$$
\begin{aligned}
\frac{D S}{D t} & =S_{\theta} \frac{D \theta}{D t}+S_{\rho} \frac{D \rho}{D t} \\
& =S_{\theta} \frac{D \theta}{D t}-\rho S_{\rho} \frac{\partial v_{k}}{\partial x_{k}} .
\end{aligned}
$$

Thus (1.3) has the form:

$$
\begin{aligned}
\frac{D \theta}{D t}= & \frac{1}{\rho \theta S_{\theta}} \frac{\partial}{\partial x_{k}}\left(\kappa \frac{\partial \theta}{\partial x_{k}}\right)+\frac{\mu}{2 \rho \theta S_{\theta}}\left(\frac{\partial v_{i}}{\partial x_{k}}+\frac{\partial v_{k}}{\partial x_{i}}\right)^{2} \\
& +\frac{\mu^{\prime}}{\rho \theta S_{\theta}}\left(\frac{\partial v_{k}}{\partial x_{k}}\right)^{2}+\frac{\rho S_{\rho}}{S_{\theta}} \frac{\partial v_{k}}{\partial x_{k}} .
\end{aligned}
$$

Hereafter, we shall consider (1.1), (1.2), (1.3)' under the following initial-boundary condition:

$$
\left\{\begin{array}{l}
v(x, 0)=v_{0}(x), \theta(x, 0)=\theta_{0}(x), \rho(x, 0)=\rho_{0}(x) \quad(x \in \Omega), \\
v(x, t)=0, \theta(x, t)=\theta_{1}(x, t) \quad\left((x, t) \in \Gamma_{T}\right) .
\end{array}\right.
$$

From now on we always assume that the compatibility conditions between the system of equations (1.1), (1.2), (1.3)' and the initialboundary condition (1.5) are satisfied.

As a final result for the problem (1.1), (1.2), (1.3)', (1.5), we have the following main theorem (as for notations, see $\S \S 1.2,1.3$ ):

Theorem 1. If $\Omega$ be a domain in $R^{3}$, bounded or undounded, and its boundary $\Gamma$ belong to $C^{2+\alpha}$ and satisfy the Lyapunov conditions, then there uniquely exists $(v, \theta, \rho) \in H^{2+\alpha}\left(\bar{Q}_{T^{\prime}}\right) \times H^{2+\alpha}\left(\bar{Q}_{T^{\prime}}\right)$ $\times B^{1+\alpha}\left(\bar{Q}_{T^{\prime}}\right)$ for some $T^{\prime} \in(0, T]$ such that $(v, \theta, 0)$ satisfies (1.1), (1.2), (1.3)', (1.5), where $\left(v_{0}, \theta_{0}, \rho_{0}\right) \in H^{2+\alpha}(\bar{\Omega}) \times H^{2+\alpha}(\bar{\Omega}) \times H^{1+\alpha}(\bar{\Omega})$ $\left(0<\bar{\rho}_{0} \equiv \inf _{x \in \bar{\Omega}} \rho_{0}(x) \leqq \rho_{0}(x) \leqq \bar{\rho}_{0} \equiv \sup _{x \in \bar{\Omega}} \rho_{0}(x)<+\infty, 0<\bar{\theta}_{0} \equiv \inf _{x \in \bar{\Omega}} \theta_{0}(x) \leqq \theta_{0}(x)\right.$ $\left.\leqq \overline{\bar{\theta}}_{0} \equiv \sup _{x \in \overline{\bar{\Omega}}} \theta_{0}(x)<+\infty\right), \theta_{1}(x, t) \in H^{2+\alpha}\left(\Gamma_{T}\right), f \in B^{1+L}\left(\bar{Q}_{T}\right)$ and $\mu, \mu^{\prime}, \kappa, p, S$ $\in \mathcal{O}_{\mathrm{loc}}^{2+L}\left(\mathscr{D}_{\rho, \theta}\right)$ with prescribed properties.

1.2. Basic Notations. For $s=\left(s_{1}, s_{2}, s_{3}\right)$ ( $s_{i}$ 's non-negative integers), $r$ (non-negative integer), we define

$$
|s|=\sum_{i=1}^{3} s_{i}, D_{t}^{r} D_{x}^{s}=\partial^{r+|s|} / \partial t^{r} \partial x_{1}^{s_{1}} \partial x_{2}^{s_{2}} \partial x_{3}^{s_{3}} .
$$

$$
Q_{T}=\Omega \times(0, T], \bar{Q}_{T}=\bar{\Omega} \times[0, T],
$$




$$
\Gamma_{T}=\Gamma \times[0, T](T \in(0,+\infty)) .
$$

For any non-negative integer $n$ and $\alpha \in(0,1)$

$$
\begin{aligned}
& H^{n+\alpha}\left(\bar{Q}_{T}\right)=\left\{\left.v(x, t)\left|\|v\|_{T}^{(n+\alpha)} \equiv \sum_{2 r+|s|=0}^{n}\right| D_{t}^{r} D_{x}^{s} v\right|_{T} ^{(0)}\right. \\
& \left.+\sum_{2 r+|s|=(n-1) \bigvee 0}\left|D_{t}^{r} D_{x}^{s} v\right|_{t, T}^{(\alpha / 2)}+\sum_{2 r+|s|=n}\left|D_{t}^{r} D_{x}^{s} v\right|_{x, T}^{(\alpha)}<+\infty\right\},
\end{aligned}
$$

where

$$
\left\{\begin{array}{l}
|v|_{T^{(0)}}^{(0)}=\sup _{(x, t) \in \bar{Q}_{T}}|v(x, t)| \\
|v|_{t, T}^{(\alpha / 2)}=\sup _{(x, t),\left(x, t^{\prime}\right) \in \bar{Q}_{T, t \neq t^{\prime}}} \frac{\left|v(x, t)-v\left(x, t^{\prime}\right)\right|}{\left|t-t^{\prime}\right|^{\alpha / 2}} \\
|v|_{x, T}^{(\alpha)}=\sup _{(x, t),\left(x^{\prime}, t\right) \in \bar{Q}_{T}, x \neq x^{\prime}} \frac{\left|v(x, t)-v\left(x^{\prime}, t\right)\right|}{\left|x-x^{\prime}\right|^{\alpha}}
\end{array}\right.
$$

$$
|v|_{T}^{(\alpha)}=|v|_{t, T}^{(\alpha / 2)}+|v|_{x, T}^{(\alpha)}
$$

(1. 10) $\quad H^{n+\alpha}(\bar{\Omega})=\left\{\left.u(x)\left|\|u\|^{(n+\alpha)} \equiv \sum_{|s|=0}^{n}\right| D_{x}^{s} u\right|^{(0)}+\sum_{|s|=n}\left|D_{x}^{s} u\right|^{(\alpha)}<+\infty\right\}$, where

$$
\begin{aligned}
& \left\{\begin{array}{l}
|u|^{(0)}=\sup _{x \in \overline{\bar{\Omega}}}|u(x)| \\
|u|^{(\alpha)}=\sup _{x, x^{\prime} \in \bar{\Omega}, x \neq x^{\prime}} \frac{\left|u(x)-u\left(x^{\prime}\right)\right|}{\left|x-x^{\prime}\right|^{\alpha}}
\end{array}\right. \\
& B^{n+\alpha}\left(\bar{Q}_{T}\right)=\left\{\left.w(x, t)\left|\sum_{r+|s|=0}^{n}\right| D_{t}^{r} D_{x}^{s} w\right|_{T} ^{(0)}\right. \\
& \left.+\sum_{r+|s|=n}\left|D_{t}^{r} D_{x}^{s}{ }^{s}\right|_{T}^{(\alpha)}<+\infty\right\} .
\end{aligned}
$$

For a vector function $g(x, t)=\left(g_{i}\right)_{i=1}^{k}, g \in H^{n+\alpha}\left(\bar{Q}_{T}\right)$ implies $g_{i}$ $\in H^{n+\alpha}\left(\bar{Q}_{T}\right) \quad(i=1,2, \cdots, k)$ and $|g|_{T}{ }^{(0)}$ denotes $\sum_{i=1}^{k}\left|g_{i}\right|_{T}{ }^{(0)}$, etc. For the Hölder exponent $\alpha=1$, notations such as $|g|_{r}{ }^{(L)}$ are used.

$$
\mathscr{D}_{\rho, \theta}=\{(\rho, \theta) \in(0,+\infty) \times(0,+\infty)\} .
$$

For $n=0,1,2, \cdots$,

(1.14) $O_{\text {loc }}^{n+L}\left(\mathscr{D}_{\rho, \theta}\right)=\left\{q(\rho, \theta) \mid q\right.$ is defined on $\mathscr{D}_{\rho, \theta^{\prime}} n$-times partially differentiable and all its $n$-th order derivatives are locally Lipschitz-continuous there\}. 
Other notations, not described above, will be explained where they appear.

1.3. On the Domain. We shall always assume that the domain in $R^{3}$, bounded or unbounded, to be considered here has a boundary which satisfies the following Lyapunov conditions (cf. [21]):

1. At each point of the boundary there exists a well-defined tangent plane and hence a well-defined normal.

2. For any point $x \in \Gamma$ there exists a single fixed number $d>0$ such that the portion of the boundary inside a sphere of radius $d$ with the center of $x$ intersects lines parallel to the normal at $x$ in at most one point.

3. If $\theta$ be the angle between the normals at $x_{1}$ and $x_{2}$, then

$$
\theta \leqq a\left|x_{1}-x_{2}\right|^{\alpha}
$$

where $a$ and $\alpha$ are positive constants independent of $x_{1}, x_{2}$ and $\alpha \leqq 1$.

The sphere mentioned above we shall call by the Lyapunov sphere.

Let $\left(\widehat{x}_{1}, \widehat{x}_{2}, \widehat{x}_{3}\right)$ be a local rectangular coordinate system with the center of $\xi \in \Gamma$, i.e., we take the inner normal at $\xi$ as the $\widehat{x}_{3}$ axis and place the $\widehat{x}_{1}, \widehat{x}_{2}$-axes in the tangent plane at $\xi$. Because of condition 2 , the portion of the boundary lying inside the Lyapunov sphere around $\xi$ may be represented in the form:

$$
\widehat{x}_{3}=F\left(\widehat{x}_{1}, \widehat{x}_{2} ; \xi\right),
$$

where $F\left(\widehat{x}_{1}, \widehat{x}_{2} ; \hat{\xi}\right)$ is a single-valued function in a certain domain of the $\left(\widehat{x}_{1}, \widehat{x}_{2}\right)$-plane. Conditions 1 and 3 imply that $F \in C^{1+\alpha}$.

We shall call that $\Gamma \in C^{m}(m>1)$ if it satisfies the above mentioned conditions and if in the neighborhood of each $\xi \in \Gamma$ it may be represented by (1.15) with $F \in C^{m}\left(K_{0}\right)$, where $K_{0}=\left\{\widehat{x}^{\prime} \equiv\left(\widehat{x}_{1}, \widehat{x}_{2}\right)|| \widehat{x}^{\prime} \mid \equiv\left(\widehat{x}_{1}{ }^{2}+\widehat{x}_{2}{ }^{2}\right)^{1 / 2}\right.$ $\leqq d / 2\}$. It is easily seen that in $K_{0}$ the function $F$ satisfies the inequalities

$$
\left\{\begin{array}{l}
|F| \leqq \beta_{1}\left|\widehat{x}^{\prime}\right|^{1+\alpha} \\
|\operatorname{grad} F| \leqq \beta_{2}\left|\widehat{x}^{\prime}\right|^{\alpha}
\end{array}\right.
$$

where $\beta_{1}$ and $\beta_{2}$ are positive constants uniformly in $\xi \in \Gamma$.

Let $\lambda$ be any small positive number. It is well known (cf. [54]) 
that in $\bar{\Omega}$ we can construct two systems $\left\{\omega^{(k)}\right\}$ and $\left\{\Omega^{(k)}\right\}$ such that

1. $\omega^{(k)} \subset \Omega^{(k)} \subset \bar{\Omega}, \bigcup_{k} \omega^{(k)}=\bigcup_{k} \Omega^{(k)}=\bar{\Omega}$,

2. for any $x$, there exists $\omega^{(k)}$ such that $x \in \omega^{(k)}$ and $\operatorname{dist}\left(x, \bar{\Omega}-\omega^{(k)}\right) \geqq \beta_{3}$ $\left(\beta_{3}>0\right)$,

3. for any $\lambda$, there exists a number $N_{0}$ independent of $\lambda$ such that $\bigcap_{k=1}^{N_{0}+1} \Omega^{(k)}=\varnothing$,

4 (i). if $\Omega^{(k)} \cap \Gamma=\varnothing$, in this case we shall denote $k=k^{\prime}$, then $\omega^{\left(k^{\prime}\right)}$ and $\Omega^{\left(k^{\prime}\right)}$ are the cubes with the same center and with the length of their edges equal to $\lambda / 2$ and $\lambda$, respectively,

4 (ii). if $\omega^{(k)} \cap \Gamma \neq \varnothing$, in this case $k=k^{\prime \prime}$, then for a local rectangular coordinate system $\{\widehat{x}\}$ with the center of some $\xi^{\left(k^{n}\right)} \in \Gamma$,

$$
\begin{aligned}
& \omega^{\left(k^{\prime \prime}\right)}=\left\{\left|\widehat{x}_{i}\right| \leqq \frac{1}{2} \beta_{4} \lambda \quad(i=1,2), 0 \leqq \widehat{x}_{3}-F\left(\widehat{x}_{1}, \widehat{x}_{2} ; \xi^{\left(k^{\prime \prime}\right)}\right) \leqq \beta_{4} \lambda\right\}, \\
& \Omega^{\left(k^{\prime \prime}\right)}=\left\{\left|\widehat{x}_{i}\right| \leqq \beta_{4} \lambda \quad(i=1,2), 0 \leqq \widehat{x}_{3}-F\left(\widehat{x}_{1}, \widehat{x}_{2} ; \xi^{\left(k^{\prime \prime}\right)}\right) \leqq 2 \beta_{4} \lambda\right\},
\end{aligned}
$$

where $F$ is a function describing the boundary $\Gamma$ in the neighborhood of $\xi^{\left(k^{\prime \prime}\right)}$ and $\beta_{4}$ is a positive constant independent of $\lambda$.

By changing the variables in such a way that

$$
\bar{x}_{i}=\widehat{x}_{i} \quad(i=1,2), \bar{x}_{3}=\widehat{x}_{3}-F\left(\widehat{x}^{\prime}\right),
$$

$\Omega^{\left(k^{\prime \prime}\right)}$ is transformed into a standard cube $K=\left\{\left|\bar{x}_{i}\right| \leqq \beta_{4} \lambda \quad(i=1,2), 0 \leqq \bar{x}_{3}\right.$ $\left.\leqq 2 \beta_{4} \lambda\right\}$, and the boundary in $\Omega^{\left(k^{\prime \prime}\right)}$ is $K^{\prime}=\left\{\left|\bar{x}_{i}\right| \leqq \beta_{4} \lambda \quad(i=1,2), \bar{x}_{3}=0\right\}$.

Furthermore, it is well known that there exist the smooth functions $\zeta^{(k)}(x)$ and $\eta^{(k)}(x)$ such that

$$
\left\{\begin{array}{l}
\zeta^{(k)}(x)=\left\{\begin{array}{lll}
1 & \text { if } & x \in \omega^{(k)} \\
0 & \text { if } & x \in \bar{\Omega}-\Omega^{(k)}
\end{array}, 0 \leqq \zeta^{(k)}(x) \leqq 1,\right. \\
\eta^{(k)}(x)=0 \text { if } \quad x \in \bar{\Omega}-\Omega^{(k)}, \sum_{k} \zeta^{(k)}(x) \eta^{(k)}(x)=1, \\
\left|D_{x}^{s} \zeta^{(k)}(x)\right| \leqq A_{1}^{(|s|)} \lambda^{-|s|},\left|D_{x}^{s} \eta^{(k)}(x)\right| \leqq A_{2}^{(|s|)} \lambda^{-|s|}
\end{array}\right.
$$

Let $\left\{\Gamma^{(j)}\right\}$ be a covering of $\Gamma$ and set $\Gamma_{T}^{(j)}=\Gamma^{(j)} \times[0, T]$. As we saw above, $\Gamma^{(j)}$ can be represented by the local coordinate system $\{\bar{x}\}$ in the same way as $K^{\prime}$. Thus the function defined on $\Gamma_{T}{ }^{(j)}$ may be considered as a function $u^{(j)}\left(\bar{x}^{\prime}, t\right)$ defined on $\widetilde{\Gamma}_{T}^{(j)}=\left\{\left|\bar{x}^{\prime}\right| \leqq \beta_{4} \lambda\right\} \times[0, T]$.

$$
H^{n+\alpha}\left(\Gamma_{T}\right)=\left\{u(x, t) \mid\|u\|_{\Gamma_{T}}^{(n+\alpha)} \equiv \sup _{j}\left\|u^{(j)}\right\|_{\widetilde{\Gamma}_{T}(j)}^{(n+\alpha)}<+\infty\right\} .
$$




\section{$\S$ 2. Preliminaries}

2. 1. Characteristic Transformation. For $v \in H^{2+\alpha}\left(\bar{Q}_{T}\right)$ with $\left.v\right|_{T_{T}}$ $=0$ we consider a system of ordinary differential equations:

$$
\left\{\begin{array}{l}
\frac{d}{d \tau} \bar{x}(\tau ; x, t)=v(\bar{x}(\tau ; x, t), \tau), \\
\bar{x}(t ; x, t)=x \quad\left(\forall(x, t) \in \bar{Q}_{T}, 0 \leqq \tau \leqq t\right) .
\end{array}\right.
$$

By the assumption that $v \in H^{2+\alpha}\left(\bar{Q}_{T}\right)$, we have a unique solution curve passing $(x, t)$ that satisfies (2.1). If we put

$$
\bar{x}(0 ; x, t)=x_{0}(x, t),
$$

then it is obvious that the transformation $(x, t) \mapsto\left(x_{0}(x, t), t_{0}=t\right)$ is a one-to-one mapping from $\bar{Q}_{T}$ onto $\bar{Q}_{T}$ and especially the boundary $\Gamma_{T}$ is transformed onto $\Gamma_{T}$. We denote the inverse transformation by $\left(x\left(x_{0}, t_{0}\right), t\right.$ $=t_{0}$ ). For any function $g(x, t)$ we define

$$
\widehat{g}\left(x_{0}, t_{0}\right)=g\left(x\left(x_{0}, t_{0}\right), t=t_{0}\right) .
$$

It is to be noted that (2.1) implies

$$
\left\{\begin{array}{l}
\frac{d}{d \tau} x\left(x_{0}, \tau\right)=\widehat{v}\left(x_{0}, \tau\right), \\
x\left(x_{0}, 0\right)=x_{0} .
\end{array}\right.
$$

Thus $x\left(x_{0}, t_{0}\right)$ is expressed in such a way that

$$
x=x_{0}+\int_{0}^{t_{0}} \widehat{v}\left(x_{0}, \tau\right) d \tau .
$$

Hence

$$
\begin{aligned}
& \frac{\partial x}{\partial x_{0}}=I+\int_{0}^{t_{0}} \widehat{v}_{x_{0}}\left(x_{0}, \tau\right) d \tau, \\
& \frac{\partial}{\partial x}=\frac{\partial x_{0}}{\partial x} \cdot \frac{\partial}{\partial x_{0}}=\left(\frac{\partial x}{\partial x_{0}}\right)^{-1} \cdot \frac{\partial}{\partial x_{0}},
\end{aligned}
$$

where $I$ is an identity matrix.

For simplicity we put

$$
\left(\frac{\partial x}{\partial x_{0}}\right)^{-1}=\left(g_{i j}\right) .
$$


The system of equations (1.1), (1.2), (1.3)' implies

$$
\begin{gathered}
\frac{\partial}{\partial t_{0}} \hat{\rho}=-\hat{\rho} g_{i j} \partial_{j} \widehat{v}_{i} \quad\left(\partial_{j}=\partial / \partial x_{0 j}\right), \\
\frac{\partial}{\partial t_{0}} \widehat{v}_{i}=\frac{1}{\hat{\rho}}\left[\left(\mu+\mu^{\prime}\right) g_{i k} g_{m s}+\mu g_{j k} g_{j s} \delta_{m i}\right] \partial_{k} \partial_{s} \widehat{v}_{m} \\
+\frac{\partial_{j} \hat{\rho}}{\hat{\rho}}\left[\mu_{\hat{\rho}}^{\prime} g_{i j} g_{k s} \partial_{s} \widehat{v}_{k}+\mu_{\hat{\rho}}\left(g_{k j} g_{k s} \partial_{s} \widehat{v}_{i}+g_{k j} g_{i s} \partial_{s} \widehat{v}_{k}\right)-p \hat{\rho} g_{i j}\right] \\
+\frac{1}{\hat{\rho}}\left[\mu^{\prime} g_{i k} \partial_{s} \widehat{v}_{m}+\mu\left(g_{m k} \partial_{s} \widehat{v}_{i}+g_{j k} \partial_{s} \widehat{v}_{j} \delta_{m i}\right)\right] \partial_{k} g_{m s} \\
+\frac{1}{\hat{\rho}}\left[\mu_{\hat{\theta}}^{\prime} g_{i j} g_{k s} \partial_{j} \hat{\theta} \partial_{s} \widehat{v}_{k}+\mu_{\hat{\theta}} g_{j k}\left(g_{j s} \partial_{s} \widehat{v}_{i}+g_{i s} \partial_{s} \widehat{v}_{j}\right) \partial_{k} \hat{\theta}\right. \\
\left.-p_{\hat{\theta}} g_{i j} \partial_{j} \hat{\theta}+f_{i}\right] \quad(i=1,2,3),
\end{gathered}
$$

$$
\begin{aligned}
\frac{\partial \hat{\theta}}{\partial t_{0}}= & \frac{\kappa}{\hat{\rho} \hat{\theta} S_{\hat{\theta}}} g_{i j} g_{i k} \partial_{j} \partial_{k} \hat{\theta}+\frac{\kappa_{\hat{\rho}}}{\hat{\rho} \hat{\theta} S_{\hat{\theta}}} g_{i j} g_{i k} \partial_{k} \hat{\theta} \partial_{j} \hat{\rho} \\
& +\frac{\kappa}{\hat{\rho} \hat{\theta} S_{\hat{\theta}}} g_{i j} \partial_{k} \hat{\theta} \partial_{j} g_{i k}+\frac{1}{\hat{\rho} \hat{\theta} S_{\hat{\theta}}}\left[\kappa_{\hat{\theta}} g_{i j} g_{i k} \partial_{j} \hat{\theta} \partial_{k} \hat{\theta}+\frac{\mu}{2}\left(g_{j k} \partial_{j} \widehat{v}_{i}\right.\right. \\
& \left.\left.+g_{i k} \partial_{k} \widehat{v}_{j}\right)^{2}+\mu^{\prime}\left(g_{i j} \partial_{j} \widehat{v}_{i}\right)^{2}+\hat{\rho}^{2} \hat{\theta} S_{\hat{\theta}} g_{i j} \partial_{j} \widehat{v}_{i}\right] .
\end{aligned}
$$

Condition (1.5) implies

$$
\left\{\begin{array}{l}
\widehat{v}\left(x_{0}, 0\right)=v_{0}\left(x_{0}\right), \hat{\theta}\left(x_{0}, 0\right)=\theta_{0}\left(x_{0}\right), \hat{\rho}\left(x_{0}, 0\right)=\rho_{0}\left(x_{0}\right) \quad\left(x_{0} \in \Omega\right), \\
\widehat{v}\left(x_{0}, t_{0}\right)=0, \hat{\theta}\left(x_{0}, t_{0}\right)=\theta_{1}\left(x_{0}, t_{0}\right) \quad\left(\left(x_{0}, t_{0}\right) \in \Gamma_{T}\right) .
\end{array}\right.
$$

Next we state the following well known fact (cf. [42]):

Lemma 2. 1. Let $\Gamma \in C^{m+\alpha}(m \geqq 0)$ and $g \in C^{m+\alpha}\left(\Gamma_{T}\right)$. Then there exists at least one function $g^{*} \in C^{m+\alpha}\left(\bar{Q}_{T}\right)$ such that $g^{*}=g$ on $\Gamma_{T}$.

Using this lemma, we can extend $\theta_{1} \in H^{2+\alpha}\left(\Gamma_{T}\right)$ to $\theta_{1}{ }^{*} \in H^{2+\alpha}\left(\bar{Q}_{T}\right)$, which we also denote by $\theta_{1}$ without misunderstanding.

2. 2. Restatement of the Problem. We shall use the notation $(x, t)$ instead of $\left(x_{0}, t_{0}\right)$ except the last section. If $\hat{v} \in H^{2+\alpha}\left(\bar{Q}_{T}\right)$ is given in (2.9), then $\hat{\rho}$ is uniquely determined, being expressed by 


$$
\hat{\rho}(x, t)=\rho_{0}(x) \cdot \exp \left[-\int_{0}^{t} g_{i j} \partial_{j} \widehat{v}_{i}(x, \tau) d \tau\right] .
$$

If in (2.10) and (2.11) we put

$$
\left\{\begin{array}{l}
w_{i}(x, t)=\widehat{v}_{i}(x, t)-v_{0 i}(x) \quad(i=1,2,3), \\
w_{4}(x, t)=\hat{\theta}(x, t)-\theta_{1}(x, t)+\theta_{1}(x, 0)-\theta_{0}(x),
\end{array}\right.
$$

then (2.10) and (2.11) can be written in the form

$$
\frac{\partial}{\partial t} w_{i}=\mathfrak{i} i_{i j}^{k m}(x, t, w) \partial_{k} \partial_{m} w_{j}+\mathfrak{B}_{i}(x, t, w) \quad(i=1,2,3,4)
$$

and the initial-boundary condition (2.12) is transformed into

$$
w(x, 0)=0,\left.w(x, t)\right|_{\Gamma_{T}}=0 .
$$

As a second main theorem we shall prove

Theorem 2. Under the same assumptions of Theorem 1, for some $T^{\prime} \in(0, T]$ there uniquely cxists $(w, \hat{\rho}) \in H^{2+\alpha}\left(\bar{Q}_{T^{\prime}}\right) \times B^{1+\alpha}\left(\bar{Q}_{T^{\prime}}\right)$ such that (w, $\rho)$ satisfies (2.9) with $w_{i}+v_{0 i}$ in place of $\widehat{v}_{i},(2.15)$ and (2.16).

First of all we proceed to prove Theorem 2 and next to prove Theorem 1 briefly.

At first we consider the following linear problem connected with (2. 15):

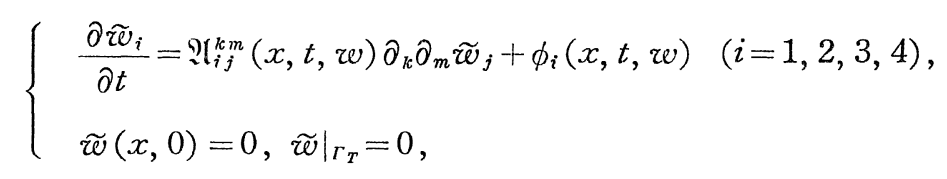

where $\phi_{i}(i=1,2,3,4)$ are any given functions which belong to $H^{\alpha}\left(\bar{Q}_{T}\right)$ and satisfy the compatibility condition. Here we assume that

$$
\begin{aligned}
& w \in \mathcal{S}_{T}=\left\{w \in H^{2+\alpha}\left(\bar{Q}_{T}\right)|w|_{T_{T}}=0,\right. \\
& \left.\langle w\rangle_{T}^{(2, \alpha)} \equiv \sum_{2 r+|s|=0}^{2}\left|D_{t}^{r} D_{x}^{s} w\right|_{T}^{(0)}+\sum_{|s|=1}\left|D_{x}^{s} w\right|_{t, T}^{(\alpha / 2)}<M_{1}\right\},
\end{aligned}
$$

where $M_{1}$ is an arbitrary positive number smaller than $\bar{\theta}_{0}$.

Let $M_{0}$ be the positive root of $1-3 x-6 x^{2}-6 x^{3}=0$. Then there 
exists $T_{1} \in(0, T]$ such that

$$
\left(M_{1}+\left\|v_{0}\right\|^{(2+\alpha)}\right) T_{1}<M_{0}, \bar{\theta}_{0}-M_{1}-\left\|\theta_{1}\right\|_{T_{1}}^{(2+\alpha)} \cdot T_{1}>0 .
$$

On this account, matrix $\left(g_{i j}\right)$ is well-defined and the following inequality holds :

$$
\left|g_{i j}-\delta_{i j}\right| \leqq B_{1}\left(\bar{T},\langle w\rangle_{\bar{T}}^{(2, \alpha)}\right) \quad\left(\bar{T} \in\left(0, T_{1}\right]\right),
$$

where $B_{1}$ is a positive constant increasing in each argument and $B_{1} \downarrow 0$ as $\bar{T} \downarrow 0$.

Lemma 2. 2. Assume (2.18). Then the system (2.17) is uniformly parabolic in the sense of Petrowsky, i.e., there exists a number o>0 such that

$$
\max _{j} \sup _{|\xi|=1} \operatorname{Re} \lambda_{j}(\xi, x, t) \leqq-\delta \quad\left(\forall(x, t) \in \bar{Q}_{T}\right),
$$

where $\lambda_{j}$ 's are the roots of $\operatorname{det}\left\{\mathfrak{U}_{s j}^{k m}\left(i \xi_{k}\right)\left(i \xi_{m}\right)-\lambda \delta_{s j}\right\}=0$.

Proof. Since

$$
\begin{aligned}
& \operatorname{det}\left\{\mathfrak{U}_{s j}^{k m}\left(i \xi_{k}\right)\left(i \xi_{m}\right)-\lambda \delta_{s j}\right\}=\left(\lambda+\frac{\mu}{\hat{\rho}} g_{j k} g_{j m} \xi_{k} \xi_{m}\right)^{2} \\
& \times\left(\lambda+\frac{2 \mu+\mu^{\prime}}{\hat{\rho}} g_{j k} g_{j m} \xi_{k} \xi_{m}\right)\left(\lambda+\frac{\kappa}{\hat{\rho} \hat{\theta} S_{\hat{\theta}}} g_{j k} g_{j m} \xi_{k} \xi_{m}\right),
\end{aligned}
$$

we have

$$
\left\{\begin{array}{l}
\lambda_{1}=\lambda_{2}=-\frac{\mu}{\hat{\rho}} g_{j k} g_{j m} \xi_{k} \xi_{m}, \\
\lambda_{3}=-\frac{2 \mu+\mu^{\prime}}{\hat{\rho}} g_{j k} g_{j m} \xi_{k} \xi_{m}, \\
\lambda_{4}=-\frac{\kappa}{\hat{\rho} \hat{\theta} S_{\hat{\theta}}} g_{j k} g_{j m} \xi_{k} \xi_{m} .
\end{array}\right.
$$

Using (2.20) we obtain

$$
\begin{aligned}
g_{j k} g_{j m} \xi_{k} \xi_{m} & \geqq \sum_{j} \xi_{j}{ }^{2} \sum_{i}\left\{g_{i j}^{2}-\sum_{k \neq j}\left|g_{i j}\right|\left|g_{i k}\right|\right\} \\
& \geqq\left(1-6 B_{1}-3 B_{1}{ }^{2}\right)|\xi|^{2} .
\end{aligned}
$$

Because of the property of $B_{1}$, there exists $T_{2} \in\left(0, T_{1}\right]$ such that 


$$
0<B_{1}\left(T_{2}, M_{1}\right)<1,1-6 B_{1}\left(T_{2}, M_{1}\right)-3 B_{1}\left(T_{2}, M_{1}\right)^{2}>0 .
$$

From (2.13), (2.14), (2.18), (2.20) and the properties of $\rho_{0}$ and $\theta_{0}$ it follows that the domain considered in (1.13) becomes

$$
\begin{aligned}
\mathscr{D}_{\hat{\rho}, \hat{\theta}}^{*}= & \left(\bar{\rho}_{0} \cdot \exp \left[-\left(M_{1}+\left\|v_{0}\right\|^{(2+\alpha)}\right) 9\left(1+B_{1}\right) T_{2}\right],\right. \\
& \left.\overline{\bar{\rho}}_{0} \cdot \exp \left[\left(M_{1}+\left\|v_{0}\right\|^{(2+\alpha)}\right) 9\left(1+B_{1}\right) T_{2}\right]\right) \\
& \times\left(\bar{\theta}_{0}-M_{1}-\left\|\theta_{1}\right\|_{T_{2}}^{(2+\alpha)} \cdot T_{2}, \overline{\bar{\theta}}_{0}+M_{1}+2\left\|\theta_{1}\right\|_{T_{2}}^{(2+\alpha)}\right) .
\end{aligned}
$$

Hence for $\sigma \in\left\{\kappa, \mu, \mu^{\prime}, S_{\hat{\theta}}\right\}$ we can define

$$
\bar{\sigma}=\min _{(\hat{\rho}, \hat{\theta}) \in \mathscr{D}^{*} \hat{\rho}, \hat{\theta}} \sigma(\hat{\rho}, \hat{\theta}), \quad \overline{\bar{\sigma}}=\max _{(\hat{\rho}, \hat{\theta}) \in \mathscr{D}^{*} \hat{\rho}, \hat{\theta}} \sigma(\hat{\rho}, \hat{\theta}) .
$$

Thus for any $(x, t) \in \bar{Q}_{T_{2}}$

$$
\begin{aligned}
& \max _{j} \sup _{|\xi|=1} \operatorname{Re} \lambda_{j}(\xi, x, t) \leqq-\left(1-6 B_{1}-3 B_{1}^{2}\right) \\
& \quad \times \min \left\{\frac{\bar{\mu}}{\overline{\bar{\rho}}_{0}}, \frac{\bar{\kappa}}{\overline{\bar{\rho}}_{0}\left(\overline{\bar{\theta}}_{0}+M_{1}+2\left\|\theta_{1}\right\|_{T_{2}}^{(2+a)}\right) \overline{\bar{S}}_{\hat{\theta}}}\right\} \\
& \quad \times \exp \left[-\left(M_{1}+\left\|v_{0}\right\|^{(2+\alpha)}\right) 9\left(1+B_{1}\right) T_{2}\right] \equiv-\delta . \quad \text { Q.E.D. }
\end{aligned}
$$

Hereafter, for simplicity we choose $T$ from the beginning in such a way that $T=T_{2}$.

\section{$\S 3$. Green Matrix of a Linear Problem}

\section{1. Some Results of a Fundamental Solution in the Whole} Space $\boldsymbol{R}^{3}$. We begin this section with a general, well-known lemma concerning the extention of functions ([19,54]):

Lemma 3. 1. Suppose $\Gamma \in C^{\tilde{m}}, \widetilde{m}=\max (1+\alpha, m)$ and $g \in H^{m}\left(\bar{Q}_{T}\right)$. Then there exists an extention * $g$ of $g$, which belongs to $H^{m}\left(\bar{R}_{T}^{3}\right)$ and satisfies the inequality $\left\|{ }^{*} g\right\|_{T, R}^{(m)} \leqq C\|g\|_{T}{ }^{(m)}$, wherc $R_{T}{ }^{3}=R^{3} \times(0, T]$ and $\left\|^{*} g\right\|_{T_{,} R^{3}}^{(m)}$ is defined by the formulas (1.7)-(1.9) with $\bar{R}_{T}{ }^{3}$ instead of $\bar{Q}_{T}$.

Henceforth we shall always denote the extention of a function $g$ by $* g$.

Since the properties of the fundamental solution in the whole space 
$R^{3}$ are well known, we state them briefly.

First of all, in connection with (2.17), we consider the following system of ordinary differential equations in $t$ :

$$
\left\{\begin{array}{l}
\frac{d V}{d t}=* \mathfrak{U}(y, \tau, w ; i \zeta) \cdot V(\zeta, t-\tau ; y, \tau ; w), \\
\left.V(\zeta, t-\tau ; y, \tau ; w)\right|_{t=\tau}=I\left(\zeta \in C^{3}\right),
\end{array}\right.
$$

where $\quad * \mathfrak{U}(y, \tau, w ; i \zeta)=\left({ }^{*} \mathfrak{H}_{s j}^{k m}(y, \tau, w(y, \tau))\left(i \zeta_{k}\right)\left(i \zeta_{m}\right)\right) . \quad V \quad$ can be solved directly from (3.1), i.e.,

$$
V(\zeta, t-\tau ; y, \tau ; w)=\exp [(t-\tau) \cdot * \mathfrak{A}(y, \tau, w ; i \zeta)]
$$

Then the parametrix $Z_{0}$ of the equation

$$
\frac{\partial u}{\partial t}=* \mathfrak{U}\left(x, t, w ; D_{x}\right) u
$$

is defined by the formula

$$
\begin{gathered}
Z_{0}(x-\xi, t-\tau ; y, \tau ; w)=(2 \pi)^{-3} \int_{R^{3}} \exp \left[i \xi_{0} \cdot(x-\xi)\right] \\
\times V\left(\xi_{0}, t-\tau ; y, \tau ; w\right) d \xi_{0} .
\end{gathered}
$$

Lemma 3. 2. ([13,18]) If $A$ be any matrix of $N$-th order with complex components whose eigenvalues are $\lambda_{1}, \cdots, \lambda_{N}$, then

$$
\|\exp [t A]\| \leqq \sum_{k=0}^{N-1}(2 t\|A\|)^{k} \cdot \exp \left[t \cdot \max _{j} \operatorname{Re} \lambda_{j}\right]
$$

Introducing the notation $|A|=\sum_{i, j}\left|A_{i j}\right|$ for the matrix $A=\left(A_{i j}\right)$, we have the following inequalities:

$$
\frac{1}{2 N^{2}}|A|^{2} \leqq \max _{j} \sum_{k}\left|A_{k j}\right|^{2} \leqq\|A\|^{2} \leqq \sum_{j, k}\left|A_{k j}\right|^{2} \leqq|A|^{2} .
$$

Since for $\zeta=\xi+i \eta\left(\xi, \eta \in R^{3}\right)$

$$
\begin{aligned}
& \operatorname{Re} \lambda_{1}(\zeta)=\operatorname{Re} \lambda_{2}(\zeta)=-\frac{\mu}{\hat{\rho}} g_{i j} g_{i k} \xi_{j} \xi_{k}+\frac{\mu}{\hat{\rho}} g_{i j} g_{i k} \eta_{j} \eta_{k} \\
& \operatorname{Re} \lambda_{3}(\zeta)=-\frac{2 \mu+\mu^{\prime}}{\hat{\rho}} g_{i j} g_{i k} \xi_{j} \xi_{k}+\frac{2 \mu+\mu^{\prime}}{\hat{\rho}} g_{i j} g_{i k} \eta_{j} \eta_{k} \\
& \operatorname{Re} \lambda_{4}(\zeta)=-\frac{\kappa}{\hat{\rho} \hat{\theta} S_{\hat{\theta}}} g_{i j} g_{i k} \xi_{j} \xi_{k}+\frac{\kappa}{\hat{\rho} \hat{\theta} S_{\hat{\theta}}} g_{i j} g_{i k} \eta_{j} \eta_{k}
\end{aligned}
$$


we have

$$
\max _{j} \operatorname{Re} \lambda_{j}(\zeta, x, t) \leqq-\delta|\xi|^{2}+B_{2}|\eta|^{2}
$$

where $\delta$ is a constant in (2.24) and

$$
\begin{aligned}
& B_{2}=(1+\left.6 B_{1}+3 B_{1}{ }^{2}\right)\left[( 2 \overline { \overline { \mu } } + \overline { \overline { \mu } } ^ { \prime } ) ( \overline { \rho } _ { 0 } ) ^ { - 1 } \overline { \overline { \kappa } } \left\{\bar{\rho}_{0}\left(\bar{\theta}_{0}-M_{1}-\left\|\theta_{1}\right\|_{T}{ }^{(2+\alpha)} \cdot T\right)\right.\right. \\
&\left.\left.\times \bar{S}_{\hat{\theta}}\right\}^{-1}\right] \cdot \exp \left[\left(M_{1}+\left\|v_{0}\right\|^{(2+\alpha)}\right) 9\left(1+B_{1}\right) T\right] .
\end{aligned}
$$

On the other hand the inequality

$$
|* \mathfrak{H}| \leqq 10 B_{2}|\zeta|^{2}
$$

holds. Hence

(3. 7) $\quad|\exp [(t-\tau) \cdot * \mathfrak{M}(y, \tau, w ; i \zeta)]| \leqq C_{0}$

$$
\times \exp \left[(t-\tau)\left\{-\frac{\delta}{2}|\xi|^{2}+\left(B_{2}+\frac{\delta}{2}\right)|\eta|^{2}\right\}\right],
$$

where $C_{0}=4 \sqrt{2}\left[1+2 K(1,0) B_{2} \delta^{-1}+4 K(2,0) B_{2}{ }^{2} \delta^{-2}+8 K(3,0) B_{2}{ }^{3} \delta^{-3}\right]$, and for $a \geqq 0, b \in[0,1), K(a, b)=\{a /(1-b) e\}^{a}$. Thus we obtain

$$
\begin{aligned}
& |V(\zeta, t-\tau ; y, \tau ; w)| \leqq C_{0} \\
& \quad \times \exp \left[\left\{-\frac{\delta}{2}|\xi|^{2}+\left(B_{2}+\frac{\delta}{2}\right)|\eta|^{2}\right\}(t-\tau)\right] .
\end{aligned}
$$

From (3.4) and (3.8) it follows that

\section{Lemma 3. 3.}

$$
\begin{aligned}
\left|D_{t}^{r} D_{x}^{s} Z_{0}\right| & \leqq C_{1}^{(r,|s|)}(t-\tau)^{-(3+2 r+|s|) / 2} \\
& \times \exp \left[-\frac{|x-\xi|^{2}}{8 \delta_{1}(t-\tau)}\right] \quad\left(\delta_{1} \equiv B_{2}+\frac{\delta}{2}\right),
\end{aligned}
$$

$$
\begin{aligned}
& \left|\Delta_{x}{ }^{\prime} D_{t}^{r} D_{x}^{s} Z_{0}\right| \leqq C_{1}^{(r,|s|+1)}\left|x-x^{\prime}\right|(t-\tau)^{-(4+2 r+|s|) / 2} \\
& \quad \times \exp \left[-\frac{\left|x^{\prime \prime}-\xi\right|^{2}}{8 \delta_{1}(t-\tau)}\right], \\
& \left(x^{\prime \prime}=x^{\prime} \text { if }\left|x^{\prime}-\xi\right| \leqq|x-\xi| ; x^{\prime \prime}=x \text {, otherwise }\right),
\end{aligned}
$$

(3. 11) $\left|\Delta_{t}^{t^{\prime}} D_{t}{ }^{r} D_{x}{ }^{s} Z_{0}\right| \leqq C_{1}{ }^{(r+1,|s|)}\left(t-t^{\prime}\right)\left(t^{\prime}-\tau\right)^{-(5+2 r+|s|) / 2}$

$$
\times \exp \left[-\frac{|x-\xi|^{2}}{8 \delta_{1}(t-\tau)}\right]\left(t>t^{\prime}>\tau\right)
$$


where we use the notations $\Delta_{x, t^{\prime}}^{x^{\prime}} g(x, t)=g(x, t)-g\left(x^{\prime}, t^{\prime}\right), \Delta_{x}^{x^{\prime}}=\Delta_{x, t}^{x^{\prime}, t}$ and $\Delta_{t}^{t^{\prime}}=\Delta_{x, t}^{x, t^{\prime}}$.

\section{Lemma 3.4.}

$$
\begin{aligned}
& \left|D_{x}^{s} Z_{0}(x-\xi, t-\tau ; y, \tau ; w)-D_{x}^{s} Z_{0}(x-\xi, t-\tau ; y+h, \tau ; w)\right| \\
& \leqq C_{2}{ }^{\left(\mid s_{i}\right)}|h|^{\alpha}(t-\tau)^{-(3+|s|) / 2} \cdot \exp \left[-\frac{|x-\xi|^{2}}{8 \delta_{2}(t-\tau)}\right],
\end{aligned}
$$

$$
\begin{aligned}
& \left|D_{x}^{s} Z_{0}(x-\xi, t-\tau ; y, \tau ; w)-D_{x}^{s} Z_{0}(x-\xi, t-\tau ; y, \tau+h ; w)\right| \\
& \quad \leqq \bar{C}_{2}{ }^{(|s|)}|h|^{\alpha / 2}(t-\tau)^{-(3+|s|) / 2} \cdot \exp \left[-\frac{|x-\xi|^{2}}{8 \delta_{2}(t-\tau)}\right], \quad\left(\delta_{2} \equiv \delta_{1}+\frac{1}{4} \delta\right) .
\end{aligned}
$$

Next the unique fundamental solution $Z(x-\xi, t-\tau ; \xi, \tau ; w)$ of (3.1) in $H^{2+\alpha}\left(\bar{R}_{T}^{3}\right)$ is defined by

$$
\begin{aligned}
& Z(x-\xi, t-\tau ; \xi, \tau ; w)=Z_{0}(x-\xi, t-\tau ; \xi, \tau ; w) \\
& +\int_{\tau}^{t} d \tau_{0} \int_{R^{3}} Z_{0}\left(x-y, t-\tau ; y, \tau_{0} ; w\right) \Phi\left(y, \tau_{0} ; \xi, \tau ; w\right) d y \equiv Z_{0}+Z^{\prime},
\end{aligned}
$$

where $\Phi$ is a solution of a Volterra type integral equation:

$$
\left\{\begin{array}{l}
\Phi(x, t ; \xi, \tau ; w)=K(x, t ; \xi, \tau ; w) \\
\quad+\int_{\tau}^{t} d \tau_{0} \int_{R^{s}} K\left(x, t ; y, \tau_{0} ; w\right) \Phi(y, \tau ; \xi, \tau ; w) d y, \\
K(x, t ; \xi, \tau ; w)=\left(* \mathfrak{N}\left(x, t, w ; D_{x}\right)\right. \\
\left.\quad-* \mathfrak{Y}\left(\xi, \tau, w ; D_{x}\right)\right) Z_{0}(x-\xi, t-\tau ; \xi, \tau ; w),
\end{array}\right.
$$

which is given by the Neumann series

$$
\left\{\begin{aligned}
\Phi(x, t ; \xi, \tau ; w)= & \sum_{m=0}^{\infty} K_{m}(x, t ; \xi, \tau ; w) \\
K_{m}(x, t ; \xi, \tau ; w)= & \int_{\tau}^{t} d \tau_{0} \int_{R^{3}} K_{0}\left(x, t ; y, \tau_{0}: w\right) \\
& \times K_{m-1}(y, \tau ; \xi, \tau ; w) d y \\
K_{0}(x, t ; \xi, \tau ; w)= & K(x, t ; \xi, \tau ; w)
\end{aligned}\right.
$$

\section{Lemma 3. 5.}




$$
\begin{aligned}
\left|D_{t}^{r} D_{x}^{s} Z^{\prime}\right| & \leqq C_{3}^{(r,|s|)}(t-\tau)^{-(3+2 r+|s|-\alpha) / 2} \\
& \times \exp \left[-\frac{d_{1}}{36} \frac{|x-\xi|^{2}}{t-\tau}\right], \quad(2 r+|s| \leqq 2),
\end{aligned}
$$

$$
\begin{aligned}
& \left|\Delta_{x}^{x^{\prime}} D_{t}^{r} D_{x}^{s} Z^{\prime}\right| \leqq C_{4}{ }^{(r,|s|)}\left|x-x^{\prime}\right|^{\alpha}(t-\tau)^{-5 / 2} \\
& \times \exp \left[-\frac{d_{1}}{72} \frac{\left|x^{\prime \prime}-\xi\right|^{2}}{t-\tau}\right],(2 r+|s|=2), \\
& \left|\Delta_{t}^{t^{\prime}} D_{t}^{r} D_{x}^{s} Z^{\prime}\right| \leqq C_{5}^{(r,|s|)}\left(t-t^{\prime}\right)^{(2-2 r-|s|+\alpha) / 2}\left(t^{\prime}-\tau\right)^{-5 / 2} \\
& \quad \times \exp \left[-\frac{d_{1}}{72} \frac{|x-\xi|^{2}}{t-\tau}\right],\left(0<2 r+|s| \leqq 2, t>t^{\prime}>\tau\right),
\end{aligned}
$$

where $d_{1}=1 / 16 \delta_{1}$.

\section{Lemma 3. 6.}

$$
\begin{aligned}
& \left|D_{t}^{r} D_{x}^{s} Z\right| \leqq C_{6}{ }^{(r,|s|)}(t-\tau)^{-(3+2 r+|s|) / 2} \\
& \times \exp \left[-\frac{d_{1}}{36} \frac{|x-\xi|^{2}}{t-\tau}\right],(2 r+|s| \leqq 2) \\
& \left|\Delta_{x}^{x^{\prime}} D_{t}^{r} D_{x}^{s} Z\right| \leqq C_{7}^{(r,|s|)}\left\{\left|x-x^{\prime}\right|^{r}(t-\tau)^{-(5+r) / 2}\right. \\
& \left.+\left|x-x^{\prime}\right|^{\beta}(t-\tau)^{-(5-\alpha+\beta) / 2}\right\} \cdot \exp \left[-\frac{d_{1}}{72} \frac{\left|x^{\prime \prime}-\xi\right|^{2}}{t-\tau}\right] \\
& \left(2 r+\left|s^{\prime}\right|=2, \gamma \in[0,1], \beta \in[0, \alpha]\right) \\
& \left|\Delta_{t}^{t^{\prime}} D_{t}^{r} D_{x}^{s} Z\right| \leqq C_{8}^{(r,|s|)}\left\{\left(t-t^{\prime}\right)\left(t^{\prime}-\tau\right)^{-(5+2 r+|s|) / 2}\right. \\
& \left.+\left(t-t^{\prime}\right)^{(2-2 r-|s|+\alpha) / 2}\left(t^{\prime}-\tau\right)^{-5 / 2}\right\} \exp \left[-\frac{d_{1}}{72} \frac{|x-\xi|^{2}}{t-\tau}\right] \\
& \left(0<2 r+|s| \leqq 2, t>t^{\prime}>\tau\right) .
\end{aligned}
$$

3. 2. The Basic Condition of Uniform Solvability. Let $\mathfrak{H}^{\left(k^{\prime \prime}\right)}\left(\xi^{\left(k^{\prime \prime}\right)}\right.$, $\left.\tau, w ; D_{\bar{x}}\right)$ be a matrix obtained from $\mathfrak{U}\left(\xi^{\left(k^{\prime \prime}\right)}, \tau, w ; D_{x}\right)$ by the transformation of the coordinate system into the local rectangular coordinate system $\{\hat{x}\}$ around $\xi^{\left(k^{\prime \prime}\right)} \in \Gamma$ and the replacement of $D_{\hat{x}}$ by $D_{\bar{x}}$. Let $\hat{\mathfrak{H}}^{\left(k^{\prime \prime}\right)}\left(\xi^{\left(k^{\prime \prime}\right)}, \tau, w ; i \bar{x}, v\right)$ be the matrix whose elements are the cofactors of $\mathfrak{P l}^{\left(k^{\prime \prime}\right)}\left(\xi^{\left(k^{\prime \prime}\right)}, \tau, w ; i \bar{x}\right)-v I$. Then we have

Lemma 3. 7. For any $\bar{x}^{\prime}=\left(\bar{x}_{1}, \bar{x}_{2}\right) \in R^{2}$ and any $v$ such that 


$$
\operatorname{Re} v \geqq-\delta_{3} \bar{x}^{\prime 2},|v|^{2}+\bar{x}^{\prime 4}>0 \quad\left(0<\delta_{3}<\tilde{\delta}\right),
$$

the row vectors of the matrix $\hat{\mathfrak{Y}}^{\left(k^{\prime \prime}\right)}\left(\xi^{\left(k^{\prime \prime}\right)}, \tau, w ; i \bar{x}, \nu\right)$ are independent modulo

$$
M^{+}=\prod_{j=1}^{4}\left(\bar{x}_{3}-\bar{x}_{3}{ }^{+(j)}\left(\bar{x}^{\prime}, v\right)\right)
$$

where $\bar{x}_{3}{ }^{+(j)}$ 's are the roots in $\bar{x}_{3}$ of $\operatorname{det}\left\{\mathfrak{P}^{\left(k^{\prime \prime}\right)}\left(\xi^{\left(k^{\prime \prime}\right)}, \tau, w ; i \bar{x}\right)-v I\right\}=0$ with positive imaginary parts, and $\widetilde{\delta}$ is a constant appearing in (2.24) with $B_{1} C_{9}$ in place of $B_{1}$ if $\left|D_{x} \widehat{x}\right| \leqq C_{9}$.

Proof. If we put $\left(g_{i j}\right)\left(D_{x} \widehat{x}\right)=\left(\widetilde{g}_{i j}\right)$, then $\mathfrak{P I}^{\left(k^{\prime \prime}\right)}\left(\xi^{\left(k^{n}\right)}, \tau, w ; i \bar{x}\right)$ is obtained from $\mathfrak{H}\left(\xi^{\left(k^{\prime \prime}\right)}, \tau, w ; i \bar{x}\right)$ by the replacement of $g_{i j}$ by $\tilde{g}_{i j}$. That is to say from (2.22) it follows that

$$
\begin{aligned}
& -\frac{\mu}{\hat{\rho}} \widetilde{g}_{i j} \widetilde{g}_{i k} \bar{x}_{j} \bar{x}_{k}=v,-\frac{2 \mu+\mu^{\prime}}{\hat{\rho}} \widetilde{g}_{i j} \widetilde{g}_{i k} \bar{x}_{j} \bar{x}_{k}=v, \\
& -\frac{\kappa}{\hat{\rho} \hat{\theta} S_{\hat{\theta}}} \widetilde{g}_{i j} \widetilde{g}_{i k} \bar{x}_{j} \bar{x}_{k}=v .
\end{aligned}
$$

Setting

(3. 26) $\left.(\mu / \hat{\rho})^{-1}=a_{1}=a_{2}, \quad\left\{\left(2 \mu+\mu^{\prime}\right) / \hat{\rho}\right)\right\}^{-1}=a_{3}, \quad\left(\kappa / \hat{\rho} \hat{\theta} S_{\hat{\theta}}\right)^{-1}=a_{4}$,

we have

$$
\widetilde{g}_{i j} \widetilde{g}_{i k} \bar{x}_{j} \bar{x}_{k}=-a_{m} v, \quad(m=1,2,3,4) .
$$

Solving this equation in $\bar{x}_{3}$, we have

$$
\begin{gathered}
\bar{x}_{3}{ }^{ \pm(m)}=\left(\widetilde{g}_{j 3}\right)^{-2}\left\{-\widetilde{g}_{k 3}\left(\bar{x}_{1}+\widetilde{g}_{k 2} \bar{x}_{2}\right) \pm\left(A_{+}{ }^{(m)}+i B_{+}{ }^{(m)}\right)\right\}, \\
(m=1,2,3,4),
\end{gathered}
$$

where

$$
\left\{\begin{array}{l}
\left.B_{+}{ }^{(m)}=\left\{\frac{1}{2}\left[\left\{\operatorname{Re} D_{m}\right)^{2}+\left(\operatorname{Im} D_{m}\right)^{2}\right\}^{1 / 2}-\operatorname{Re} D_{m}\right]\right\}^{1 / 2}(>0), \\
A_{+}{ }^{(m)}=\operatorname{Im} D_{m} / 2 B_{+}{ }^{(m)}, \\
D_{m}=-\frac{1}{2}\left\{\left(\widetilde{g}_{j 3} \widetilde{g}_{k 1}-\widetilde{g}_{k 3} \widetilde{g}_{j 1}\right) \bar{x}_{1}+\left(\widetilde{g}_{j 3} \widetilde{g}_{k 2}-\widetilde{g}_{k s} \widetilde{g}_{j 2}\right) \bar{x}_{2}\right\}^{2}-a_{m}\left(\widetilde{g}_{j 3}\right)^{2} v .
\end{array}\right.
$$

Therefore $M^{+}$defined by (3.24) is expressed by

$$
M^{+}=\left(\bar{x}_{3}-\bar{x}_{3}^{+(1)}\right)^{2}\left(\bar{x}_{3}-\bar{x}_{3}^{+(3)}\right)\left(\bar{x}_{3}-\bar{x}_{3}{ }^{+(4)}\right) .
$$


The matrix $\hat{\mathfrak{P}}^{\left(k^{\prime \prime}\right)}\left(\xi^{\left(k^{\prime \prime}\right)}, \tau, w ; i \bar{x}, v\right)=\left(\hat{\mathfrak{U}}_{i j}^{\left(k^{\prime \prime}\right)}\right)$ may be written as follows:

$$
\begin{aligned}
& \left(-\frac{\left(\widetilde{g}_{m 3}\right)^{6}}{a_{1} a_{3} a_{4}}\left(\bar{x}_{3}-\bar{x}_{3}{ }^{+(1)}\right)\left(\bar{x}_{3}-\bar{x}_{3}{ }^{-(1)}\right)\left(\bar{x}_{3}-\bar{x}_{3}{ }^{+(3)}\right)\left(\bar{x}_{3}-\bar{x}_{3}{ }^{-(3)}\right)\right. \\
& \times\left(\bar{x}_{3}-\bar{x}_{3}{ }^{+(4)}\right)\left(\bar{x}_{3}-\bar{x}_{3}{ }^{-(4)}\right) \delta_{i j}+\frac{\left(\widetilde{g}_{m 3}\right)^{4}}{a_{1} a_{4} a_{5}}\left(\widetilde{g}_{i 1} \bar{x}_{1}+\widetilde{g}_{i 2} \bar{x}_{2}\right. \\
& \left.+\widetilde{g}_{i 3} \bar{x}_{3}\right)\left(\widetilde{g}_{j 1} \bar{x}_{1}+\widetilde{g}_{j 2} \bar{x}_{2}+\widetilde{g}_{j 3} \bar{x}_{3}\right)\left(\bar{x}_{3}-\bar{x}_{3}{ }^{+(1)}\right)\left(\bar{x}_{3}-\bar{x}_{3}{ }^{(1)}\right)
\end{aligned}
$$

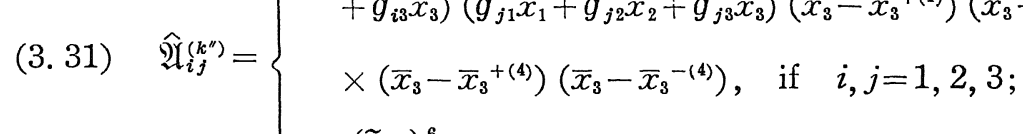

$$
\begin{aligned}
& -\frac{\left(\widetilde{g}_{m 3}\right)^{6}}{a_{1}^{2} a_{3}}\left(\bar{x}_{3}-\bar{x}_{3}{ }^{+(1)}\right)^{2}\left(\bar{x}_{3}-\bar{x}_{3}{ }^{-(1)}\right)^{2}\left(\bar{x}_{3}-\bar{x}_{3}{ }^{+(3)}\right) \\
& \times\left(\bar{x}_{3}-\bar{x}_{3}{ }^{-(3)}\right), \quad \text { if } \quad i=j=4 ; \\
& 0 \text {, otherwise ; }
\end{aligned}
$$

where $a_{5} \equiv\left\{\left(\mu+\mu^{\prime}\right) / \hat{\rho}\right\}^{-1}$. Let $\breve{\mathfrak{A}}_{i j}^{\left(k^{\prime \prime}\right)}$ be the remainder when we divide $\hat{\mathfrak{N}}_{i j}^{\left(k^{\prime \prime}\right)}$ by $M^{+}$. Then

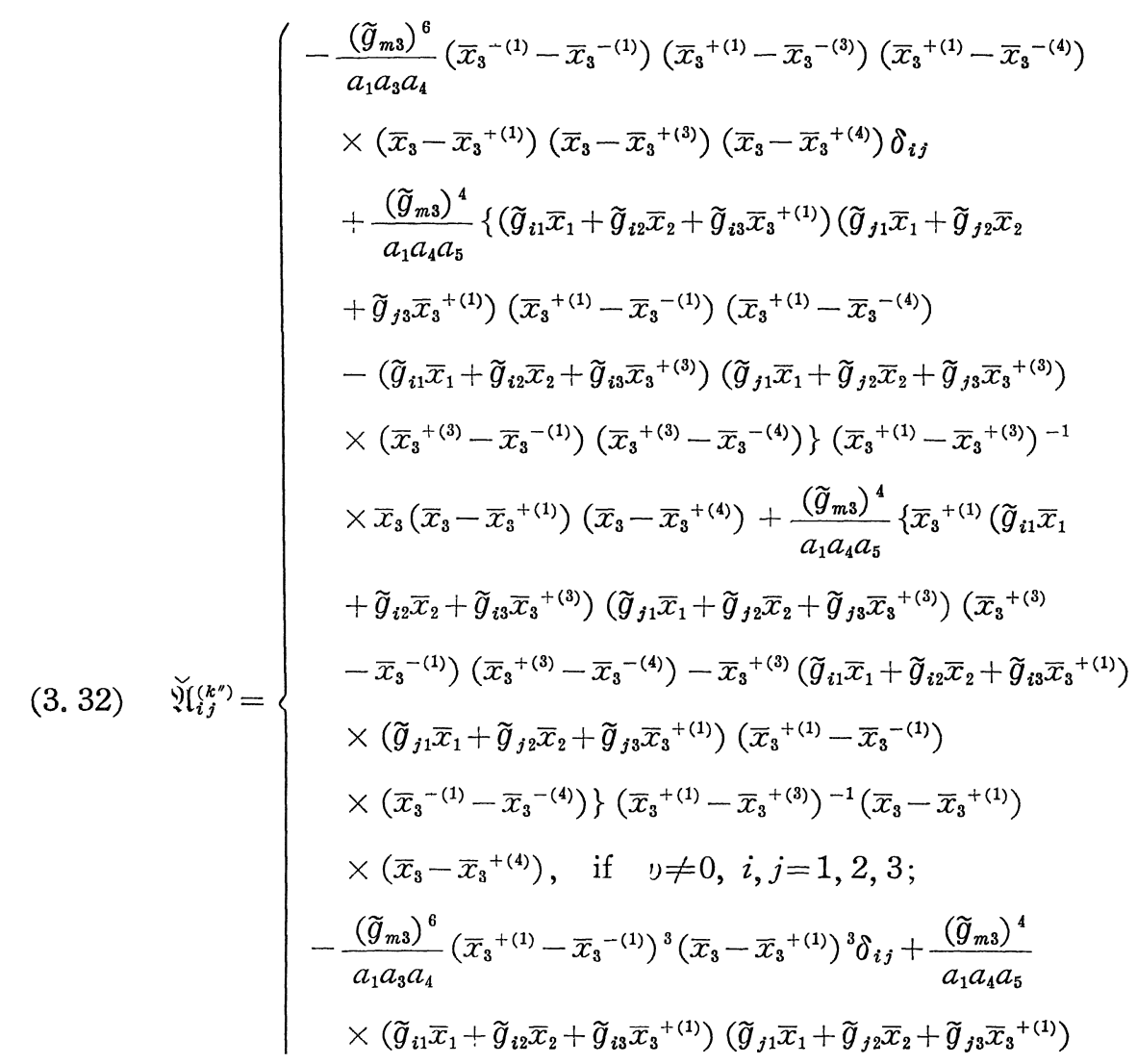




$$
\mid \begin{aligned}
& \quad \times\left(\bar{x}_{3}{ }^{+(1)}-\bar{x}_{3}{ }^{-(1)}\right)^{2}\left(\bar{x}_{3}-\bar{x}_{3}{ }^{+(1)}\right)^{2}, \text { if } v=0, i, j=1,2,3 ; \\
& -\frac{\left(\widetilde{g}_{m 3}\right)^{6}}{a_{1}{ }^{2} a_{3}}\left(\bar{x}_{3}{ }^{+(4)}-\bar{x}_{3}^{-(1)}\right)^{2}\left(\bar{x}_{3}{ }^{+(4)}-\bar{x}_{3}^{-(3)}\right)\left(\bar{x}_{3}-\bar{x}_{3}{ }^{+(1)}\right)^{2} \\
& \quad \times\left(\bar{x}_{3}-\bar{x}_{3}{ }^{+(3)}\right), \text { if } i=j=4 ; \\
& 0, \text { otherwise. }
\end{aligned}
$$

(N.B. If $v=0$, then $\bar{x}_{3}{ }^{+(1)}=\bar{x}^{+(3)}=\bar{x}_{3}{ }^{+(4)}, \bar{x}_{3}^{-(1)}=\bar{x}_{3}{ }^{-(3)}=\bar{x}_{3}{ }^{-(4)}$.) If we put $\breve{\mathfrak{U}}_{i j}^{\left(k^{\prime \prime}\right)}=\sum_{s=1}^{4} \alpha_{i j}^{(s)} \bar{x}_{3}{ }^{s-1}$, then in order to show that the row vectors of $\breve{\mathfrak{U}}^{\left(k^{\prime \prime}\right)}$ are linearly independent as polynomials of $\bar{x}_{3}$, it is sufficient to show that the rank of the matrix $\left(\alpha_{i j}^{(s)}\right)$ is 4 for any $\bar{x}^{\prime} \in R^{2}$ and $v$ satisfying (3.23). From (3.32) it follows that

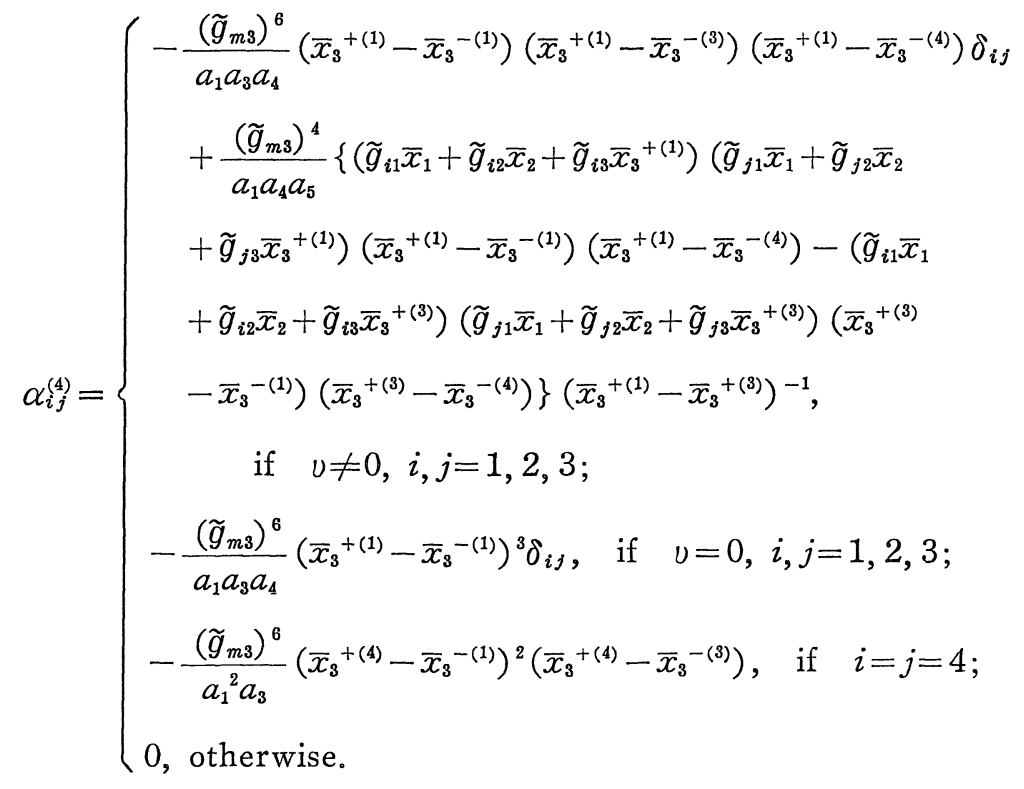

Now let us consider $\operatorname{det}\left(\alpha_{i j}^{(4)}\right) \equiv \Delta$. By $(3.33)$

(3. 34) $\Delta=\left\{\quad-\left(\widetilde{g}_{i 1} \bar{x}_{1}+\widetilde{g}_{i 2} \bar{x}_{2}+\widetilde{g}_{i 3} \bar{x}_{3}{ }^{+(3)}\right)^{2}\left(\bar{x}_{3}{ }^{+(3)}-\bar{x}_{3}{ }^{-(1)}\right)\left(\bar{x}_{3}{ }^{+(3)}-\bar{x}_{3}{ }^{-(4)}\right)\right\}$ 


$$
\left\{\begin{array}{l}
-\frac{1}{a_{5}^{2}}\left\{\left(\widetilde{g}_{i 1} \widetilde{g}_{j 3}-\widetilde{g}_{j 1} \widetilde{g}_{i 3}\right) \bar{x}_{1}+\left(\widetilde{g}_{i 2} \widetilde{g}_{j 3}-\widetilde{g}_{j 2} \widetilde{g}_{i 3}\right) \bar{x}_{2}\right\}^{2} \\
\left.\times\left(\bar{x}_{3}^{+(3)}-\bar{x}_{3}^{-(4)}\right)\left(\bar{x}_{3}^{+(1)}-\bar{x}_{3}^{+(3)}\right)\right]\left(\bar{x}_{3}^{+(1)}-\bar{x}_{3}^{+(3)}\right)^{-1} \\
\text { if } \quad v \neq 0 ; \\
\frac{\left(\widetilde{g}_{m 3}\right)^{24}}{a_{1}{ }^{5} a_{3}{ }^{2} a_{4}{ }^{3}}\left(\bar{x}_{3}{ }^{+(1)}-\bar{x}_{3}^{-(1)}\right)^{12}, \text { if } v=0 .
\end{array}\right.
$$

In the case of $v=0$, it is clearly by (3.34) that $\Delta \neq 0$. Next in the case of $v \neq 0$, since by (3.28) and (3.29)

$$
\begin{aligned}
\left(\bar{x}_{3}{ }^{+(1)}-\bar{x}_{3}^{-(3)}\right)\left(\bar{x}_{3}^{+(1)}-\bar{x}_{3}^{+(3)}\right) & =\left(\widetilde{g}_{m 3}\right)^{-4}\left[D_{1}-D_{3}\right] \\
& =\left(a_{3}-a_{1}\right)\left(\widetilde{g}_{m 3}\right)^{-2},,
\end{aligned}
$$

and by (3.26) and the definition of $a_{5}$ in (3.31)

$$
\frac{a_{3}-a_{1}}{a_{3}^{2}}+\frac{a_{1}}{a_{3} a_{5}}=0
$$

we have for the product of the last two factors in (3.34), $\Delta_{1}$,

$$
\begin{aligned}
\Delta_{1}=- & \frac{1}{a_{5}}\left(x_{3}{ }^{+(3)}-x_{3}{ }^{-(4)}\right)\left[( a _ { 3 } - a _ { 1 } ) ^ { - 1 } \left\{A_{+}{ }^{(1)}+A_{+}{ }^{(3)}\right.\right. \\
& \left.+i\left(B_{+}{ }^{(1)}+B_{+}{ }^{(3)}\right)\right\}^{2}+a_{5}{ }^{-1}\left\{\left(\widetilde{g}_{i 1} \widetilde{g}_{j 3}-\widetilde{g}_{j 1} \widetilde{g}_{i 3}\right) \bar{x}_{1}\right. \\
& \left.\left.+\left(\widetilde{g}_{i 2} \widetilde{g}_{j 3}-\widetilde{g}_{j 2} \widetilde{g}_{i 3}\right) \bar{x}_{2}\right\}^{2}\right] \neq 0 .
\end{aligned}
$$

Hence $\Delta \neq 0$, i.e., $\operatorname{rank}\left(\alpha_{j k}^{(s)}\right)=4$.

Q.E.D.

3. 2. On the Poisson Kernel. Making use of the fundamental solution $Z$ constructed in $\S 3.1$, we shall find a Green matrix $G$ for a system (2.17) of the form

$$
G=Z-G_{0},
$$

where $G_{0}$ is a solution of a system of equations:

$$
\left\{\begin{array}{l}
D_{t} W=\mathfrak{U}\left(x, t, w ; D_{x}\right) W, \\
\left.W\right|_{t=\tau}=0,\left.W\right|_{\Gamma_{\tau}, T}=\left.Z\right|_{\Gamma_{r}, T}(0 \leqq \tau \leqq t \leqq T) .
\end{array}\right.
$$

Here we use the notations

$$
\mathfrak{U}\left(x, t, w ; D_{x}\right)=\left(\mathfrak{H}_{i j}^{k m} \partial_{k} \partial_{m}\right), \Gamma_{\tau, T}=\Gamma \times[\tau, T] .
$$


In connection with (3.39), we consider the following system of equations :

$$
\left\{\begin{array}{l}
D_{t} W=\mathfrak{A}\left(x, t, w ; D_{x}\right) W \text { in } Q_{\tau, \tau+h} \equiv \Omega \times(\tau, \tau+h], \\
\left.W\right|_{t=\tau}=0,\left.W\right|_{\Gamma_{\tau}, \tau+h}=\left.Z(x-\xi, t-\tau ; \xi, \tau ; w)\right|_{\Gamma_{\tau, \tau+h}},
\end{array}\right.
$$

where $(\xi, \tau)$ is an arbitrary fixed point in $Q_{T}$ and $0<h \leqq T-\tau$.

For simplicity the transformation from $x$ to $\widehat{x}[$ resp. $\bar{x}]$ is denoted by $\Pi_{x}^{\hat{x}}\left[\operatorname{resp} . \Pi_{x}^{\bar{x}}\right]$ and the inverse transformation, $\Pi_{\hat{x}}{ }^{x}\left[\mathrm{resp} . \Pi_{\bar{x}}{ }^{x}\right]$. Moreover the following notations are used.

$$
\begin{aligned}
& \bar{f}(\bar{x})=\Pi_{x}^{\bar{x}} f(x), \hat{f}(\hat{x})=\Pi_{x}^{\hat{x}} f(x), \bar{f}(\bar{x}, \hat{\xi})=\Pi_{x, \hat{\xi}}^{\bar{x}, \hat{\xi}} f(x, \hat{\xi}), \\
& \hat{f}(\hat{x}, \hat{\xi})=\Pi_{x, \hat{x}}^{\hat{x}, \hat{\xi}} f(x, \xi), \Pi_{x, \xi}^{\bar{x}, \hat{\xi}}=\Pi_{x}^{\bar{x}} \Pi_{\xi}^{\hat{\xi}} \text { etc. }
\end{aligned}
$$

The regularizer $R$ of

$$
\left\{\begin{array}{l}
D_{t} W=\mathfrak{U}\left(x, t, w ; D_{x}\right) W+\psi \\
\left.W\right|_{t=\tau}=0,\left.W\right|_{r_{\mathrm{r}, \tau+h}}=g
\end{array}\right.
$$

introduced in [5] can be defined by the formulas:

$$
\left\{\begin{array}{l}
W(x, t)=R(\psi, g) \equiv \sum_{k} \eta^{(k)}(x) W^{(k)}(x, t), \\
W^{\left(k^{\prime}\right)}(x, t)=R^{\left(k^{\prime}\right)} \psi \equiv \int_{\tau}^{t} d \tau_{0} \int_{\Omega\left(k^{\prime}\right)} Z_{0}\left(x-y, t-\tau_{0} ; \xi^{\left(k^{\prime}\right)}, \tau_{0} ; w\right) \\
\quad \times \zeta^{\left(k^{\prime}\right)}(y) \psi\left(y, \tau_{0}\right) d y, \\
W^{\left(k^{\prime \prime}\right)}(x, t)=\Pi_{\bar{x}}^{x} \bar{W}^{\left(k^{\prime \prime}\right)}(\bar{x}, t), \\
\bar{W}^{\left(k^{\prime \prime}\right)}(\bar{x}, t)=\bar{R}^{\left(k^{\prime \prime}\right)} \psi+\bar{R}_{1}^{\left(k^{\prime \prime}\right)} g, \\
\bar{R}^{\left(k^{\prime \prime}\right)} \psi \equiv \int_{\tau}^{t} d \tau_{0} \int_{K} H_{0}^{\left(k^{\prime \prime}\right)}\left(\bar{x}, t ; \bar{y}, \tau_{0}\right) \bar{\zeta}^{\left(k^{\prime \prime}\right)}(\bar{y}) \bar{\psi}\left(\bar{y}, \tau_{0}\right) d \bar{y}, \\
\bar{R}_{1}^{\left(k^{\prime \prime}\right)} g \equiv \int_{\tau}^{t} d \tau_{0} \int_{K^{\prime}} H_{1}^{\left(k^{\prime \prime}\right)}\left(\bar{x}-\bar{y}^{\prime}, t-\tau_{0}\right) \bar{\zeta}^{\left(k^{\prime \prime}\right)}\left(\bar{y}^{\prime}\right) \bar{g}\left(\bar{y}^{\prime}, \tau_{0}\right) d \bar{y}^{\prime},
\end{array}\right.
$$

where $H_{0}{ }^{\left(k^{\prime \prime}\right)}\left(\bar{x}, t ; \bar{y}, \tau_{0}\right)$ and $H_{1}{ }^{\left(k^{\prime \prime}\right)}\left(\bar{x}-\bar{y}^{\prime}, t-\tau_{0}\right)$ are called the Green matrix and the Poisson kernel, respectively, of the system

$$
\left\{\begin{array}{l}
D_{t} \bar{W}=\mathfrak{H}^{\left(k^{\prime \prime}\right)}\left(\xi^{\left(k^{\prime \prime}\right)}, \tau, w ; D_{\bar{x}}\right) \bar{W}+\bar{\zeta}^{\left(k^{\prime \prime}\right)}(\bar{x}) \bar{\phi}(\bar{x}, t) \\
\left.\bar{W}\right|_{t=\tau}=0,\left.\quad \bar{W}\right|_{\bar{x}_{3}=0}=\bar{\zeta}^{\left(k^{\prime \prime}\right)}\left(\bar{x}^{\prime}\right) \bar{g}\left(\bar{x}^{\prime}, t\right) .
\end{array}\right.
$$

First of all, let us construct the Poisson kernel. Using the nota- 
tions in $\S 3.2$, we define $\alpha_{s}^{(j, k)}(j, k, s=1,2,3,4)$ such that

$$
\left\{\begin{array}{c}
\alpha_{s}^{(j, k)}=0 \quad(s=1,2,3 ; j, k=1,2,3,4), \\
\sum_{j=1}^{4} \alpha_{4}^{(j, k)} \alpha_{i, j}^{(4)}=\delta_{i k} \quad(i, k=1,2,3,4) .
\end{array}\right.
$$

$m_{i}$ 's are defined by the formula

$$
M^{+}=\sum_{i=0}^{4} m_{i} \bar{x}_{3}^{4-i},
$$

i.e., from (3.30) it follows that

$$
\left\{\begin{array}{l}
m_{0}=1, m_{1}=-\left(2 \bar{x}_{3}^{+(1)}+\bar{x}_{3}^{+(3)}+\bar{x}_{3}{ }^{+(4)}\right), \\
m_{2}=\bar{x}_{3}^{+(1) 2}+2 \bar{x}_{3}^{+(1)}\left(\bar{x}_{3}^{+(3)}+\bar{x}_{3}^{+(4)}\right)+\bar{x}_{3}^{+(3)} \bar{x}_{3}{ }^{+(4)}, \\
m_{3}=-\bar{x}_{3}^{+(1) 2}\left(\bar{x}_{3}^{+(3)}+\bar{x}_{3}^{+(4)}\right)-2 \bar{x}_{3}{ }^{+(1)} \bar{x}_{3}{ }^{+(3)} \bar{x}_{3}{ }^{+(4)}, \\
m_{4}=\bar{x}_{3}^{+(1) 2} \bar{x}_{3}^{+(3)} \bar{x}_{3}^{+(4)} .
\end{array}\right.
$$

We define

$$
\left\{\begin{array}{l}
M_{k}{ }^{+} \equiv \sum_{i=0}^{k} m_{i} \bar{x}_{3}{ }^{k-1}, \\
N_{j k} \equiv \sum_{s=1}^{4} \alpha_{s}^{(j, k)} M_{4-s}^{+}, \\
\widetilde{H}_{1}^{\left(k^{\prime \prime}\right)}\left(\bar{x}, \tau_{0}\right)=\frac{1}{2 \pi i} \int_{r_{+}} \hat{\mathfrak{X}}^{\left(k^{\prime \prime}\right)}\left(\xi^{\left(k^{\prime \prime}\right)}, \tau, w ; i \bar{x}^{\prime}, i \xi, \tau_{0}\right) \\
\quad \times N\left(\bar{x}^{\prime}, \xi, \tau_{0}\right) \frac{\exp \left[i \bar{x}_{3} \cdot \xi\right]}{M^{+}\left(\bar{x}^{\prime}, \xi, \tau_{0}\right)} d \xi,
\end{array}\right.
$$

where $N=\left(N_{j k}\right)$ and $\gamma_{+}$is a contour enclosing all $\bar{x}_{3}{ }^{+(m)}(m=1,2,3,4)$.

On account of $(3.31)-(3.33),(3.44)-(3.46), \tilde{H}_{1}^{\left(k^{\prime \prime}\right)}$ can be expressed explicitly as follows:

$$
\left(\begin{array}{l}
-\frac{\left(\widetilde{g}_{n 3}\right)^{6}}{a_{1} a_{3} a_{4}}\left(\bar{x}_{3}^{+(1)}-\bar{x}_{3}^{-(1)}\right)\left(\bar{x}_{3}^{+(1)}-\bar{x}_{3}^{-(3)}\right) \\
\quad \times\left(\bar{x}_{3}^{+(1)}-\bar{x}_{3}^{-(4)}\right) \alpha_{4}^{(j, m)} \cdot \exp \left(i \bar{x}_{3}^{+(1)} \bar{x}_{3}\right) \\
\quad+\frac{\left(\widetilde{g}_{n 3}\right)^{4}}{a_{1} a_{4} a_{5}} \sum_{s=1}^{3} \alpha_{4}^{(j, s)}\left\{\left(\widetilde{g}_{s 1} \bar{x}_{1}+\widetilde{g}_{s 2} \bar{x}_{2}+\widetilde{g}_{s 3} \bar{x}_{3}^{+(1)}\right)\right. \\
\quad \times\left(\widetilde{g}_{m 1} \bar{x}_{1}+\widetilde{g}_{m 2} \bar{x}_{2}+\widetilde{g}_{m 3} \bar{x}_{3}^{+(1)}\right)\left(\bar{x}_{3}^{+(1)}-\bar{x}_{3}^{-(1)}\right) \\
\quad \times\left(\bar{x}_{3}{ }^{+(1)}-\bar{x}_{3}^{-(4)}\right)-\left(\widetilde{g}_{s 1} \bar{x}_{1}+\widetilde{g}_{s 2} \bar{x}_{2}+\widetilde{g}_{s 3} \bar{x}_{3}{ }^{+(3)}\right)
\end{array}\right.
$$




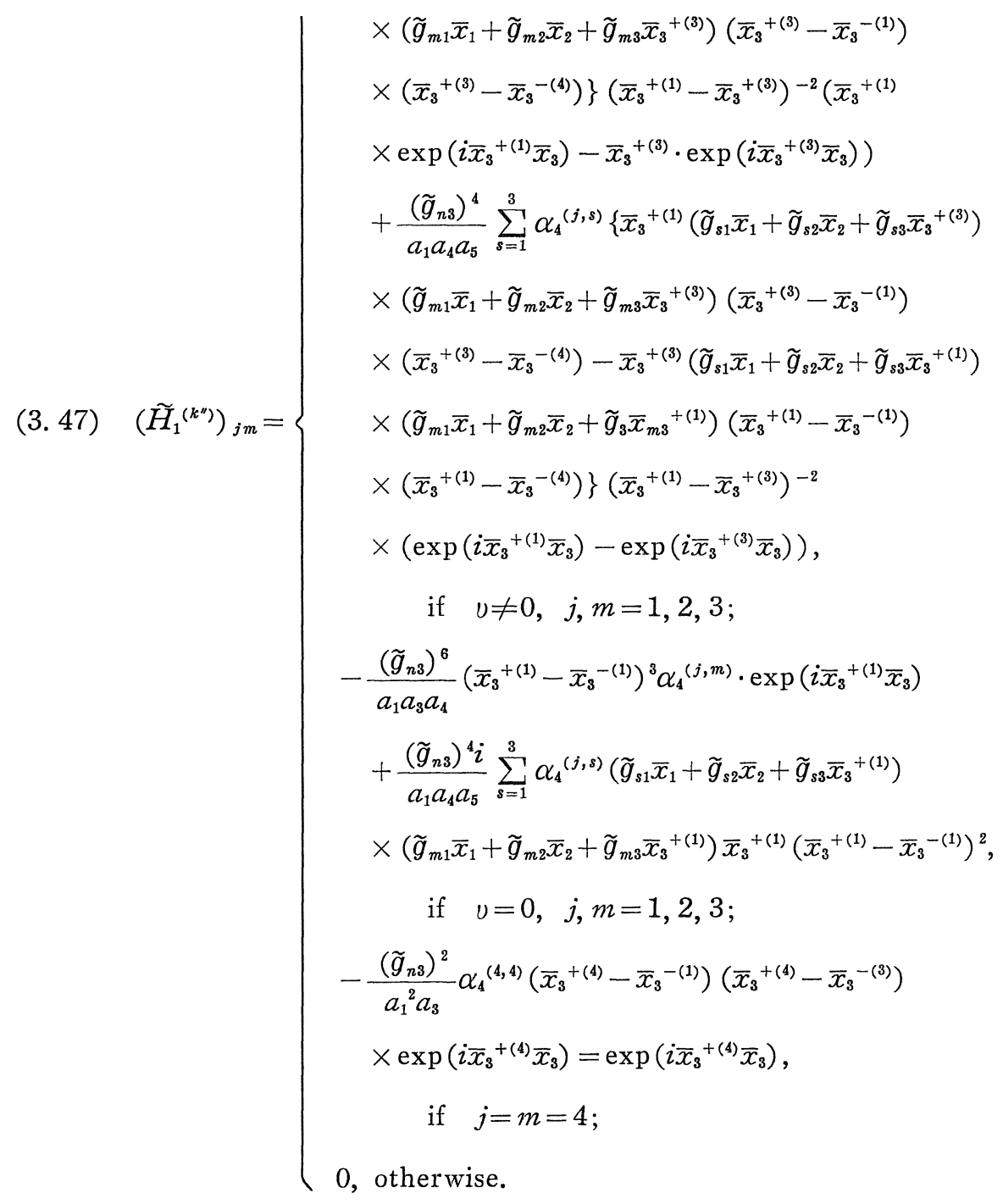

Then $H_{1}{ }^{\left(k^{\prime \prime}\right)}$ is defined by

$$
\begin{aligned}
& H_{1}^{\left(k^{\prime \prime}\right)}(\bar{x}, t)=\frac{1}{(2 \pi)^{3} i} \int_{R^{2}} \exp \left(i\left(\bar{x}^{\prime}, \zeta\right)\right) d \zeta \\
& \quad \times \int_{\sigma-i \infty}^{\sigma+i \infty} \exp \left(\tau_{0} t\right) \widetilde{H}_{1}^{\left(k^{\prime \prime}\right)}\left(\zeta, \bar{x}_{3}, \tau_{0}\right) d \tau_{0}\left(\sigma>-\delta_{3} \zeta^{2}\right) .
\end{aligned}
$$

Hence the same arguments as used above imply that the Green matrix of (3.43) is defined by

$$
H_{0}{ }^{\left(k^{\prime \prime}\right)}(\bar{x}, t ; \bar{y}, \tau)=Z_{0}{ }^{\left(k^{\prime \prime}\right)}\left(\bar{x}-\bar{y}^{\prime}, t-\tau ; \xi^{\left(k^{\prime \prime}\right)}, \tau ; w\right)
$$




$$
-\int_{\tau}^{t} d \tau_{0} \int_{R^{2}} H_{1}{ }^{\left(k^{\prime \prime}\right)}\left(\bar{x}-\bar{y}_{0}{ }^{\prime}, t-\tau_{0}\right) Z_{0}{ }^{\left(k^{\prime \prime}\right)}\left(\bar{y}_{0}{ }^{\prime}-\bar{y}, \tau_{0}-\tau ; \xi^{\left(k^{\prime \prime}\right)}, \tau ; w\right) d \bar{y}_{0}{ }^{\prime},
$$

where $Z_{0}^{\left(k^{\prime \prime}\right)}$ is a fundamental solution of (3.43).

Now let us put

$$
\left\{\begin{array}{l}
q=v+\delta_{3} \bar{x}^{\prime 2}\left(0<\delta_{3}<\tilde{\delta} ; \delta_{3}, \text { fixed }\right), \\
A^{\left(k^{\prime \prime}\right)} \equiv \operatorname{det}\left\{\mathfrak{\mathfrak { X } ^ { ( k ^ { \prime \prime } ) }}\left(\xi^{\left(k^{\prime \prime}\right)}, \tau, w ; i \bar{x}\right)-\left(q-\delta_{3} \bar{x}^{2}\right) I\right\} .
\end{array}\right.
$$

Then the following lemma clearly holds.

Lemma 3. 8. For any $\bar{x}^{\prime} \in R^{2}$ and any $q \in C^{1}$ with $\operatorname{Re} q>0$, $A^{\left(k^{\prime \prime}\right)}=0$ in $\bar{x}_{3}$ has four roots $\bar{x}_{3}^{+(j)}$ with $\operatorname{Im} \bar{x}_{3}^{+(j)}>0$ and four $\bar{x}_{3}^{-(j)}$ with $\operatorname{Im} \bar{x}_{3}^{-(j)}<0$.

Since $q_{j}$ and $\bar{x}_{3}{ }^{ \pm(j)}$ have homogeneous properties, i.e.,

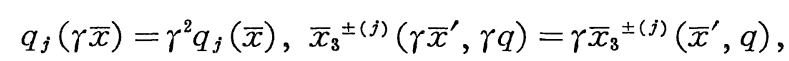

where $q_{j}$ is the root of $A^{\left(k^{\prime \prime}\right)}=0$ with respect to $q$,

$$
\left|q_{j}(\bar{x})\right| \leqq C_{10}|\bar{x}|^{2},\left|\bar{x}_{3}^{ \pm(j)}\right| \leqq C_{11}\left(\left|\bar{x}^{\prime}\right|^{4}+|q|^{2}\right)^{1 / 4} .
$$

$$
\operatorname{Im} \bar{x}_{3}{ }^{ \pm(j)} \geqq C_{12}\left(\left|\bar{x}^{\prime}\right|^{4}+|q|^{2}\right)^{1 / 4},
$$

hence $\left|\bar{x}_{3}^{+(j)}\right| \geqq C_{12}\left(\left|\bar{x}^{\prime}\right|^{4}+|q|^{2}\right)^{1 / 4}$ for $\bar{x}^{\prime} \in R^{2}, q \in C^{1}$ with $\operatorname{Re} q>0$.

Lemma 3.9. There exists a positive constant $\beta_{5}$ such that if $q \in C^{1}$ and $\bar{x}^{\prime} \in R^{2}$ satisfy $\operatorname{Re} q \geqq-\beta_{5}|\operatorname{Im} q|$ and $|q|^{2}+\left|\bar{x}^{\prime}\right|^{4}>0$, then $A^{\left(k^{\prime \prime}\right)}$ $=0$ has four roots with positive imaginary parts and four with negative ones.

Proof. We have only to take $\beta_{5}=\frac{1}{2}\left(\tilde{\delta}-\delta_{3}\right) C_{10}^{-1}$ because of the parabolicity condition and (3.51).

Q.E.D.

For the sake of simplicity we assume that (3.51) and (3.52) hold for $\left(\bar{x}^{\prime}, q\right)$ stated in lemma 3.9 with the same constants $C_{10}-C_{12}$.

Lemma 3. 10. There exists a positive constant $\beta_{6}$ such that 
$A^{\left(k^{\prime \prime}\right)}=0$ in $\bar{x}_{3}$ with $\bar{x}^{\prime}=\xi^{\prime}+i \eta^{\prime}\left(\xi^{\prime}, \eta^{\prime} \in R^{2}\right)$ has no real roots if (3. 53) $\quad \operatorname{Re} q \geqq-\beta_{5}|\operatorname{Im} q|,\left|\xi^{\prime}\right|^{4}+|q|^{2}>0,\left|\eta^{\prime}\right| \leqq \beta_{6}\left(\left|\xi^{\prime}\right|^{4}+|q|^{2}\right)^{1 / 4}$.

Proof. Referring to (3.28) and (3.29) it is sufficient to find $\beta_{6}(>0)$ such that

$$
\begin{gathered}
\left\{\left(\operatorname{Re} \bar{D}_{m}\right)^{2}+\left(\operatorname{Im} \bar{D}_{m}\right)^{2}\right\}^{1 / 2}-\operatorname{Re} \bar{D}_{m}-2\left\{\widetilde{g}_{j 3}\left(\widetilde{g}_{j 1} \eta_{1}+\widetilde{g}_{j 2} \eta_{2}\right)\right\}^{2}>0 \\
\left(\eta^{\prime} \equiv\left(\eta_{1}, \eta_{2}\right)\right)
\end{gathered}
$$

where

$$
\begin{aligned}
& \bar{D}_{m}=-\frac{1}{2}\left\{\left(\widetilde{g}_{j 3} \widetilde{g}_{k 1}-\widetilde{g}_{k 3} \widetilde{g}_{j 1}\right)\left(\xi_{1}+i \eta_{1}\right)+\left(\widetilde{g}_{j 2} \widetilde{g}_{k 3}-\widetilde{g}_{k 2} \widetilde{g}_{j 3}\right)\right. \\
&\left.\times\left(\xi_{2}+i \eta_{2}\right)\right\}^{2}-a_{m}\left(\widetilde{g}_{j 3}\right)^{2}\left[q-\delta_{3}\left\{\left(\xi_{1}+i \eta_{1}\right)^{2}+\left(\xi_{2}+i \eta_{2}\right)^{2}\right\}\right] \\
&\left(\xi^{\prime} \equiv\left(\xi_{1}, \xi_{2}\right)\right) .
\end{aligned}
$$

Denoting the left-hand side of (3.54) by $G(\eta)$, we have by Lemma 3.9

$$
G(0) \geqq 2 C_{12}^{2}\left(\left|\xi^{\prime}\right|^{4}+|q|^{2}\right)^{1 / 2} .
$$

Utilizing the mean value theorem and the estimate

$$
\begin{gathered}
\left|G^{\prime}(\eta)\right| \leqq 36\left(1+B_{1}\right)^{2}\left\{3\left(1+B_{1}\right)^{2}+\bar{a} \delta_{3}\right\}\left(\left|\xi^{\prime}\right|+\left|\eta^{\prime}\right|\right) \\
\left(\bar{a} \equiv \max \left(a_{1}, a_{4}\right)\right)
\end{gathered}
$$

we have only to take

$$
\beta_{6}=\min \left\{\frac{1}{4}\left[-1+\left\{1+\frac{2 C_{12}^{2}}{9\left(1+B_{1}\right)^{2}\left\{3\left(1+B_{1}\right)^{2}+\bar{a} \delta_{3}\right\}}\right\}^{1 / 2}\right], 1\right\} .
$$

Q.E.D.

It is easily seen that Lemmas $3.8-3.10$ imply

Lemma 3. 11. $\bar{x}_{3}^{ \pm(k)}$ defined by (3.28) with prescribed replacements are analytic with respect to $\bar{x}^{\prime}$ and $q$ in the domain (3.53), and (3.51) and (3.52) hold with suitable constants $\bar{C}_{i}(i=10,11,12)$.

Lemma 3. 12. There exist positive constants $\beta_{7}\left(\leqq \beta_{5}\right)$ and $\beta_{8}\left(\leqq \beta_{6}\right)$ such that $\Delta\left(\bar{x}^{\prime}, q\right) \neq 0\left(\bar{x}^{\prime}=\xi^{\prime}+i \eta^{\prime}\right)$ for $\bar{x}^{\prime}$ and $q$ satisfying 
(3. 58) $\quad \operatorname{Re} q \geqq-\beta_{7}|\operatorname{Im} q|,\left|\xi^{\prime}\right|^{4}+|q|^{2}>0,\left|\eta^{\prime}\right| \leqq \beta_{8}\left(\left|\xi^{\prime}\right|^{4}+|q|^{2}\right)^{1 / 4}$.

Proof. First of all, we consider the case $\eta^{\prime}=0$. From Lemma 3.9 it follows that for $\beta_{7}=\min \left(\beta_{5}, \frac{1}{\sqrt{6}}\right)$ and some positive constant $C_{13}$

$$
\begin{aligned}
\left|\Delta_{1}\right|^{2}= & a_{5}{ }^{-2}\left(\bar{x}_{3}{ }^{+(3)}-\bar{x}_{3}{ }^{-(4)}\right)^{2}\left\{\left[( a _ { 1 } - a _ { 3 } ) ^ { - 1 } \left\{\left(B_{+}{ }^{(1)}+B_{+}{ }^{(3)}\right)^{2}\right.\right.\right. \\
& \left.-\left(A_{+}{ }^{(1)}+A_{+}{ }^{(3)}\right)^{2}\right\}+a_{5}{ }^{-1}\left\{\left(\widetilde{g}_{i 1} \widetilde{g}_{j 3}-\widetilde{g}_{j 1} \widetilde{g}_{i 3}\right) \xi_{1}\right. \\
& \left.\left.+\left(\widetilde{g}_{i 2} \widetilde{g}_{j 3}-\widetilde{g}_{j 2} \widetilde{g}_{i 3}\right) \xi_{2}\right\}^{2}\right]^{2}+4\left(a_{1}-a_{3}\right)^{-2}\left(A_{+}{ }^{(1)}+A_{+}{ }^{(3)}\right)^{2} \\
& \left.\times\left(B_{+}{ }^{(1)}+B_{+}{ }^{(3)}\right)^{2}\right\} \geqq C_{13}\left(\left|\xi^{\prime}\right|^{4}+|q|^{2}\right) .
\end{aligned}
$$

Next in the same method as those used in the proof of Lemma 3.10 we obtain $\beta_{8}=C_{13} / 2 C_{14}$, where

$$
\left\{\begin{aligned}
C_{14}= & 32\left[\left(a_{1}-a_{3}\right)^{-1}\left(\bar{C}_{11}^{2}+C_{15}^{2} \bar{C}_{12}^{-2}\right)+a_{5}{ }^{-1} C_{15}^{2}\right]\left[\left(a_{1}-a_{3}\right)^{-1}\right. \\
& \left.\times\left\{\bar{C}_{11} \bar{C}_{12}^{-1} C_{15}+4 \bar{C}_{12}^{-2} C_{15}^{2}\left(1+\bar{C}_{12}^{-2} C_{15}\right)\right\}+2 a_{5}{ }^{-1} C_{15}\right] \\
& +16\left[2\left(a_{1}-a_{3}\right)^{-1} \bar{C}_{11} \bar{C}_{12}^{-1} C_{15}+a_{5}^{-1} C_{15}\right] \cdot\left[2\left(a_{1}-a_{3}\right)^{-1}\right. \\
& \left.\times\left\{2 \bar{C}_{11} \bar{C}_{12} C_{15}\left(1+\bar{C}_{12}^{-2} C_{15}\right)+\bar{C}_{12}^{-2} C_{15}^{2}\right\}+2 a_{5}^{-1} C_{15}\right] \\
C_{15}= & 36\left(1+B_{1}\right)^{4}+9 \bar{a}\left(1+B_{1}\right)^{2}\left(1+\delta_{3}\right) .
\end{aligned}\right.
$$

From Lemmas 3.8-3.12 and the formula (3.47) follows

Lemma 3. 13. $\tilde{H}_{2}^{\left(k^{\prime \prime}\right)}(\bar{x}, q)=\widetilde{H}_{1}{ }^{\left(k^{\prime \prime}\right)}\left(\bar{x}, q-\delta_{3} \bar{x}^{\prime 2}\right)$ is analytic with respect to $\bar{x}^{\prime}$ and $q$ in the domain defined by (3.58) and

$$
D_{\bar{x}_{3}}^{s} \widetilde{H}_{2}^{\left(k^{\prime \prime}\right)}\left(\alpha \bar{x}^{\prime}, \bar{x}_{3}, \alpha^{2} q\right)=\alpha^{s} D_{\bar{x}_{3}}^{s} \tilde{H}_{2}{ }^{\left(k^{\prime \prime}\right)}\left(\bar{x}^{\prime}, \alpha \bar{x}_{3}, q\right) .
$$

As is well known in [54] (cf. [4]) $\tilde{H}_{2}{ }^{\left(k^{\prime \prime}\right)}$ can be represented in the following form

$$
\widetilde{H}_{2}{ }^{\left(k^{\prime \prime}\right)}\left(\bar{x}^{\prime}, \bar{x}_{3}, q\right)=\hat{\mathfrak{N}}_{1}{ }^{\left(k^{\prime \prime}\right)}\left(\xi^{\left(k^{\prime \prime}\right)}, \tau, w ; i \bar{x}^{\prime}, \frac{d}{d \bar{x}_{3}}, q\right) V\left(\bar{x}^{\prime}, \bar{x}_{3}, q\right),
$$

where

$$
\begin{gathered}
\hat{\mathfrak{I}}_{1}^{\left(k^{\prime \prime}\right)}\left(\xi^{\left(k^{\prime \prime}\right)}, \tau, w ; i \bar{x}, q\right)=\hat{\mathfrak{H}}^{\left(k^{\prime \prime}\right)}\left(\xi^{\left(k^{\prime \prime}\right)}, \tau, w ; i \bar{x}, q-\delta_{3} \bar{x}^{\prime 2}\right), \\
V\left(\bar{x}^{\prime}, \bar{x}_{3}, q\right)=\mathfrak{P}_{1}{ }^{\left(k^{\prime \prime}\right)}\left(\xi^{\left(k^{\prime \prime}\right)}, \tau, w ; i \bar{x}^{\prime}, \frac{d}{d \bar{x}_{3}}, q\right) W\left(\bar{x}^{\prime}, \bar{x}_{3}, q\right),
\end{gathered}
$$




$$
\mathfrak{U}_{1}{ }^{\left(k^{\prime \prime}\right)}\left(\xi^{\left(k^{\prime \prime}\right)}, \tau, w ; i \bar{x}, q\right)=\mathfrak{Y}^{\left(k^{\prime \prime}\right)}\left(\xi^{\left(k^{\prime \prime}\right)}, \tau, w ; i \bar{x}\right)-\left(q-\delta_{3} \bar{x}^{\prime 2}\right) I \text {. }
$$

$$
\begin{aligned}
W(\bar{x}, q)=\frac{1}{2 \pi} & {\left[\int_{0}^{\bar{x}_{3}} \widetilde{H}_{2}{ }^{\left(k^{\prime \prime}\right)}\left(\bar{x}^{\prime}, z, q\right) d z \int_{r_{+}} \frac{\exp \left[i y\left(\bar{x}_{3}-z\right)\right]}{A^{\left(k^{\prime \prime}\right)}\left(i \bar{x}^{\prime}, i y, q\right)} d y\right.} \\
& \left.-\int_{\bar{x}_{3}}^{\infty} \widetilde{H}_{2}{ }^{\left(k^{\prime \prime}\right)}\left(\bar{x}^{\prime}, z, q\right) d z \int_{r_{-}} \frac{\exp \left[i y\left(\bar{x}_{3}-z\right)\right]}{A^{\left(k^{\prime \prime}\right)}\left(i \bar{x}^{\prime}, i y, q\right)} d y\right],
\end{aligned}
$$

$\left(\gamma_{-}\right.$, a contour enclosing the roots of $A^{\left(k^{\prime \prime}\right)}=0$ which belong to the lower half space).

Note that $W$ is a solution of

$$
\left\{\begin{array}{l}
A^{\left(k^{\prime \prime}\right)}\left(i \bar{x}^{\prime}, \frac{d}{d \bar{x}_{3}}, q\right) W\left(\bar{x}^{\prime}, \bar{x}_{3}, q\right)=\widetilde{H}_{2}^{\left(k^{\prime \prime}\right)}\left(\bar{x}^{\prime}, \bar{x}_{3}, q\right), \\
W \rightarrow 0 \text { as } \bar{x}_{3} \rightarrow \infty
\end{array}\right.
$$

and has the following properties:

$$
\left\{\begin{aligned}
& D_{\bar{x}_{3}}^{s} W\left(\alpha \bar{x}^{\prime}, \bar{x}_{3}, \alpha^{2} q\right)=\alpha^{s-8} D_{\bar{x}_{3}}^{s} W\left(\bar{x}^{\prime}, \alpha \bar{x}_{3}, q\right), \\
&\left|D_{\bar{x}_{3}}^{s} W(\bar{x}, q)\right| \leqq C_{16}\left(\left|\bar{x}^{\prime}\right|^{4}+|q|^{2}\right)^{(s-8) / 2} \\
& \times \exp \left[-\frac{1}{4} \bar{C}_{12} \bar{x}_{3}\left(\left|\bar{x}^{\prime}\right|^{4}+|q|^{2}\right)^{1 / 4}\right]
\end{aligned}\right.
$$

for $\forall s \geqq 0$ and $\forall\left(\bar{x}^{\prime}, q\right)$ satisfying (3.58).

As for $V$ we have for $\forall\left(\bar{x}^{\prime}, q\right)$ satisfying (3.58) and $\forall s \geqq 0$

$$
\left\{\begin{array}{r}
D_{\bar{x}_{3}}^{s} V\left(\alpha \bar{x}^{\prime}, \bar{x}_{3}, \alpha^{2} q\right)=\alpha^{s \sim 6} D_{\bar{x}_{3}}^{s} V\left(\bar{x}^{\prime}, \alpha \bar{x}_{3}, q\right) \\
\left|D_{\bar{x}_{3}}^{s} V\right| \leqq C_{17}\left(\left|\bar{x}^{\prime}\right|^{4}+|q|^{2}\right)^{(s-6) / 4} \\
\times \exp \left[-\frac{1}{4} \bar{C}_{12} \bar{x}_{3}\left(\left|\bar{x}^{\prime}\right|^{4}+|q|^{2}\right)^{1 / 4}\right] .
\end{array}\right.
$$

In (3. 48) if we put $\sigma=-\delta_{3} \zeta^{2}+a(a>0)$ and $q=\tau_{0}+\delta_{3} \zeta^{2}$, then

$$
\begin{aligned}
H_{1}\left(k^{\prime \prime}\right)(\bar{x}, t)= & \frac{1}{(2 \pi)^{3} i} \int_{R^{2}} \exp \left(i\left(\bar{x}^{\prime}, \zeta\right)-\delta_{3} \zeta^{2} t\right) d \zeta \\
& \times \int_{a-i \infty}^{a+i \infty} \exp (q t) \widetilde{H}_{2}{ }^{\left(k^{\prime \prime}\right)}\left(\zeta, \bar{x}_{3}, q\right) d q .
\end{aligned}
$$

By (3.61) we obtain

$$
H_{1}^{\left(k^{\prime \prime}\right)}(\bar{x}, t)=\hat{\mathfrak{I}}^{\left(k^{\prime \prime}\right)}\left(\xi^{\left(k^{\prime \prime}\right)}, \tau, w ; D_{\bar{x}}, D_{t}\right) K(\bar{x}, t),
$$

where 


$$
\begin{array}{r}
K(\bar{x}, t)=\frac{1}{(2 \pi)^{3} i} \int_{R^{2}} \exp \left(i\left(\bar{x}^{\prime}, \zeta\right)-\hat{o}_{3} \zeta^{2} t\right) d \zeta \\
\times \int_{a-i \infty}^{a+i \infty} \exp (q t) V\left(\zeta, \bar{x}_{3}, q\right) d q
\end{array}
$$

In connection with $K$, we define for non-negative integer $v$

$$
\begin{aligned}
K^{(v)}(\bar{x}, t)= & \frac{1}{(2 \pi)^{3} i} \int_{R^{2}} \exp \left(i\left(\bar{x}^{\prime}, \zeta\right)-\delta_{3} \zeta^{2} t\right) d \zeta \\
& \times \int_{a-i \infty}^{a+i \infty} \exp (q t)\left(q+\zeta^{2}\right)^{-v} V\left(\zeta, \bar{x}_{3}, q\right) d q
\end{aligned}
$$

From these it follows at once that the following formula holds.

$$
K(\bar{x}, t)=\left[D_{t}-\left(1+\delta_{3}\right) \Delta_{(2)}\right] K^{(v)}(\bar{x}, t),
$$

where $\Delta_{(2)} \equiv D_{\bar{x}_{1}}^{2}+D_{\bar{x}_{2}}^{2}$.

\section{Lemma 3. 14.}

$$
\left|D_{t}^{r} D_{\bar{x}}^{s} K^{(v)}(\bar{x}, t)\right| \leqq C_{18}^{(r,|s|)} t^{-(2 r+|s|-2-2 v) / 2} \cdot \exp \left[-2 d_{2} \frac{|\bar{x}|^{2}}{t}\right]
$$

Proof.

$$
\begin{aligned}
D_{t}^{r} D_{\bar{x}^{s}}^{s} K^{(\nu)}= & \frac{1}{(2 \pi)^{3} i} \int_{R^{2}} \exp \left(i\left(\bar{x}^{\prime}, \zeta\right)-\delta_{3} \zeta^{2} t\right)(i \zeta)^{s^{\prime}} d \zeta \\
& \times \int_{a-i \infty}^{a+i \infty} \exp (q t) \cdot \frac{\left(q-\delta_{3} \zeta^{2}\right)^{r}}{\left(q+\zeta^{2}\right)^{v}} D_{\bar{x}_{3}}^{s_{3}} V\left(\zeta, \bar{x}_{3}, q\right) d q \\
= & \frac{1}{(2 \pi)^{3} i} t^{-(2 r+|s|-2-2 v) / 2} \int_{R^{2}} \exp \left(i\left(\bar{y}^{\prime}, \xi\right)-\delta_{3} \xi^{2}\right)(i \xi)^{s^{\prime}} d \xi \\
& \times \int_{R^{2}} \exp (q) \frac{\left(q-\delta_{3} \xi^{2}\right)^{r}}{\left(q+\xi^{2}\right)^{v}} D_{\bar{x}_{3}}^{s_{3}} V\left(\xi, \bar{y}_{3}, q\right) d q \\
\equiv & t^{-(2 r+|s|-2-2 \nu) / 2} \cdot H_{3}(\bar{y}), \quad\left(s=\left(s_{1}, s_{2}, s_{3}\right)=\left(s^{\prime}, s_{3}\right)\right)
\end{aligned}
$$

because $D_{\bar{x}_{3}}^{s_{3}} V$ is analytic and (3.66) holds.

At first let us estimate $H_{3}(\bar{y})$. Utilizing Cauchy's integral theorem and changing the variables from $\xi$ and $q$ to $\xi \bar{y}_{3}$ and $q \bar{y}_{3}{ }^{2}$ respectively we have 
(3. 73)

$$
\begin{aligned}
H_{3}(\bar{y})= & \frac{1}{(2 \pi)^{3} i} \int_{R^{2}} \exp \left(i\left(\bar{y}^{\prime}, \xi\right)-\delta_{3} \xi^{2}\right)(i \xi)^{s^{\prime}} d \xi \\
& \times \int_{L(a)} e^{q}\left(q-\delta_{3} \zeta^{2}\right)^{r}\left(q+\xi^{2}\right)^{-v} D_{\bar{y}_{3}}^{s_{3}} V\left(\xi, \bar{y}_{3}, q\right) d q \\
= & \frac{1}{(2 \pi)^{3} i} \bar{y}^{2 r+|s|-2-2 v} \int_{R^{2}} \exp \left(i\left(\bar{y}^{\prime}, \xi\right)-\delta_{3} \xi^{2} \bar{y}_{3}{ }^{2}\right)(i \xi)^{s^{\prime}} d \xi \\
& \times \int_{L(a)} \exp \left(q \bar{y}_{3}{ }^{2}\right)\left(q-\delta_{3} \xi^{2}\right)^{r}\left(q+\xi^{2}\right)^{-v} D_{\bar{y}_{3}}^{s_{3}} V\left(\xi, \bar{y}_{3}{ }^{2}, q\right) d q,
\end{aligned}
$$

where $L(a)$ is a contour $\operatorname{Re} q=-\beta_{7}|\operatorname{Im} q|+a$. On $L(a)$

$$
\left|q+\xi^{2}\right|^{2} \geqq \frac{1}{2}\left(|q|^{2}+\xi^{4}\right) .
$$

If we define

$$
M=\max \left\{0, \frac{2 r-2 v-6+s_{3}}{2}\right\}
$$

then

$$
\left(q-\delta_{3} \xi^{2}\right)^{r}\left(q+\xi^{2}\right)^{-v}\left(|q|^{2}+\xi^{4}\right)^{\left(s_{3}-6\right) / 4} \leqq C_{19}\left(|q|^{2}+\xi^{4}\right)^{M},
$$

where

$$
C_{19}=2^{(r / 2)+v}\left(1+\delta_{3}\right)^{r / 2}\left\{1+\left[a\left(1+\beta_{7}{ }^{2}\right)^{-1 / 2}\right]^{\min \left\{\left(2 r-2 v-6+\delta_{3}\right) / 2,0\right\}}\right\} .
$$

Hence

$$
\begin{aligned}
& \left|\int_{L(a)} \exp \left(q \bar{y}_{3}{ }^{2}\right)\left(q-\delta_{3} \xi^{2}\right)^{r}\left(q+\xi^{2}\right)^{-v} D_{\bar{y}_{3}}^{s_{3}} V\left(\xi, \bar{y}_{3}{ }^{2}, q\right) d q\right| \\
& \leqq 2^{M-1} C_{17} C_{19}\left(1+\beta_{7}{ }^{2}\right)^{1 / 4}\left\{\left(a^{2}+\xi^{4}\right)^{M} \beta_{7}{ }^{-1} \bar{y}_{3}{ }^{-2}+\Gamma(2 M+1)\right. \\
& \left.\quad \times \beta_{7}{ }^{-2 M-1} \bar{y}_{3}{ }^{-4 M-2}\right\} \cdot \exp \left[\left\{a-\frac{1}{4} \bar{C}_{12} a^{1 / 2}\left(1+\beta_{7}{ }^{2}\right)^{-1 / 4}\right\} \bar{y}_{3}{ }^{2}\right] .
\end{aligned}
$$

Here we can choose $a>0$ such that

$$
a-\frac{1}{8} \bar{C}_{12} a^{1 / 2}\left(1+\beta_{7}{ }^{2}\right)^{-1 / 4}<0 .
$$

Thus we obtain

$$
\left|H_{3}(\bar{y})\right| \leqq C_{20}\left(1+{\bar{y}_{3}}^{2 r+2 v-6+s_{3}}\right) \exp \left[-\frac{1}{8} \bar{C}_{12} a^{1 / 2}\left(1+{\beta_{7}}^{2}\right)^{-1 / 4} \bar{y}_{3}{ }^{2}\right] .
$$

We can also estimate in (3.74) as follows 
$(3.77)$

$$
\begin{aligned}
& \left|\int_{L(a)} \exp \left(q \bar{y}_{3}^{2}\right)\left(q-\delta_{3} \xi^{2}\right)^{r}\left(q+\xi^{2}\right)^{-\nu} D_{\bar{y}_{3}}^{s_{3}} V\left(\xi, \bar{y}_{3}^{2}, q\right) d q\right| \\
& \quad \leqq 2^{M-1}\left(1+\beta_{7}^{2}\right)^{1 / 4} C_{17} C_{19}\left\{\left(a^{2}+\xi^{4}\right)^{M} \beta_{7}{ }^{-1}+\Gamma(2 M+1) \beta_{7}{ }^{-2 M-1}\right\}
\end{aligned}
$$

therefore

$$
\left|H_{3}(\bar{y})\right| \leqq C_{21} .
$$

From (3.76) and (3.78) follows

$$
\left|H_{3}(\bar{y})\right| \leqq C_{22} \cdot \exp \left[-\frac{1}{16} \bar{C}_{12} a^{1 / 2}\left(1+\beta_{7}{ }^{2}\right)^{-1 / 4} \bar{y}_{3}{ }^{2}\right],
$$

where

$$
C_{22}=\left[\max \left\{2 C_{20}, C_{21}\right\}\right] \cdot \exp \left[\frac{1}{8} \bar{C}_{11} a^{1 / 2}\left(1+\beta_{7}{ }^{2}\right)^{-1 / 4}\right]
$$

Next in (3.73) if we use $\xi\left|\bar{y}^{\prime}\right|$ and $q\left|\bar{y}^{\prime}\right|^{2}$ in place of $\xi$ and $q$ respectively and proceed the same arguments as above, we have

$$
\left|H_{3}(\bar{y})\right| \leqq C_{23} \cdot \exp \left[-\frac{1}{2} \gamma \beta_{8} a^{1 / 2}\left(1+\beta_{7}{ }^{2}\right)^{-1 / 4}\left|\bar{y}^{\prime}\right|^{2}\right]
$$

provided that

$$
a+\delta_{3} \gamma^{2}{\beta_{8}}^{2} a\left(1+\beta_{7}{ }^{2}\right)^{-1 / 2}-\frac{1}{2} \gamma \beta_{8} a^{1 / 2}\left(1+\beta_{7}{ }^{2}\right)^{-1 / 4}<0,
$$

where $\gamma$ is a sufficiently small positive number.

We choose a such that both (3.75) and (3.81) hold and then fix it. Therefore (3.79) and (3.80) imply that

$$
\left|H_{3}(\bar{y})\right| \leqq C_{24} \cdot \exp \left[-2 d_{2}|\bar{y}|^{2}\right] \text {. }
$$

Thus from (3.72) and (3.82) follows (3.71) with $C_{18}^{(r,|s|)}=C_{24}$.

Q.E.D.

By (3.67), (3.70) and (3.71) we can easily obtain

\section{Lemma 3. 15.}

$$
\left|D_{t}^{r} D_{\bar{x}}^{s} H_{1}^{\left(k^{\prime \prime}\right)}(\bar{x}, t)\right| \leqq C_{25}^{(r,|s|)} t^{-(4+2 r+|s|) / 2} \cdot \exp \left[-2 d_{2} \frac{|\bar{x}|^{2}}{t}\right]
$$

$$
\left|\Delta_{\bar{x}}{ }^{\bar{x}_{0}} D_{t}^{r} D_{\bar{x}}^{s} H_{1}{ }^{\left(k^{\prime \prime}\right)}(\bar{x}, t)\right| \leqq C_{25}^{(r,|s|+1)}\left|\bar{x}-\bar{x}_{0}\right| t^{-(5+2 r+|s|) / 2}
$$




$$
\begin{aligned}
& \times \exp \left[-2 d_{2} \frac{\left|\bar{x}^{\prime \prime}\right|^{2}}{t}\right], \\
& \left(\bar{x}^{\prime \prime}=\bar{x} \text { if }|\bar{x}| \leqq\left|\bar{x}_{0}\right| ; \bar{x}^{\prime \prime}=\bar{x}_{0} \text {, otherwise }\right),
\end{aligned}
$$

(3. 85) $\left|\Delta_{t}^{t_{0}} D_{t}^{r} D_{\bar{x}}^{s} H_{1}^{\left(k^{\prime \prime}\right)}(\bar{x}, t)\right| \leqq C_{25}^{(r+1,|s|)}\left(t-t_{0}\right) t_{0}{ }^{-(6+2 r+|s|) / 2}$

$$
\times \exp \left[-2 d_{2} \frac{|\bar{x}|^{2}}{t}\right],\left(t>t_{0}>0\right) .
$$

3. 4. Construction of Green Matrix. We shall find the solution of (3.40) in the form of $G_{0}=R u$ where $u=\left(u_{0}, u_{1}\right)$ and $R$ is a regularizer defined by (3.42). $u$ will be a solution of

$$
u=P u+g, \quad g=\left(0, Z_{\Gamma_{\tau, \tau+h}}\right),
$$

where $P$ is a bounded operator on $H^{2+\alpha}\left(Q_{\tau, \tau+h}\right) \times H^{2+\alpha}\left(\Gamma_{\tau, \tau+h}\right)$ when $h$ is small.

We go into more detail. $u_{0}$ and $u_{1}$ satisfy

$$
u_{0}=P_{0} u, \quad u_{1}=\left.Z\right|_{\Gamma_{r}, \tau+h},
$$

where

$$
\left\{\begin{aligned}
P_{0} u= & \sum_{k^{\prime \prime}} \eta^{\left(k^{\prime \prime}\right)}(x) \Pi_{\bar{x}, \xi}^{x, \xi}\left[\mathfrak{H} \mathfrak{H}^{\left(k^{\prime \prime}\right)}\left(\xi^{\left(k^{\prime \prime}\right)}, \tau, w ; D_{\bar{x}}-\operatorname{grad} F D_{\bar{x}_{s}}\right)\right. \\
& \left.-\mathfrak{H}^{\left(k^{\prime \prime}\right)}\left(\xi^{\left(k^{\prime \prime}\right)}, \tau, w ; D_{\bar{x}}\right)\right] \bar{U}_{k^{\prime \prime}}(\bar{x}, t ; \hat{\xi}, \tau)+\sum_{k} \eta^{(k)}(x) \\
& \times\left[\mathfrak{I}\left(x, t, w ; D_{x}\right)-\vartheta \mathfrak{Y}\left(\xi^{(k)}, \tau, w ; D_{x}\right)\right] U_{k}(x, t ; \xi, \tau) \\
& +\sum_{k}\left[\mathfrak{H}\left(x, t, w ; D_{x}\right) \eta^{(k)}(x)-\eta^{(k)}(x) \mathfrak{U}\left(x, t, w ; D_{x}\right)\right] \\
& \times U_{k}(x, t ; \hat{\xi}, \tau), \\
U_{k^{\prime}}= & R^{\left(k^{\prime}\right)} u_{0}, \\
U_{k^{\prime \prime}}= & \Pi_{\bar{x}, \hat{\xi}}^{x, \xi} \bar{U}_{k^{\prime \prime}}(\bar{x}, t ; \hat{\xi}, \tau), \\
\bar{U}_{k^{\prime \prime}}= & \bar{R}^{\left(k^{\prime \prime}\right)} u_{0}+\bar{R}_{1}^{\left(k^{\prime \prime}\right)} u_{1}, \\
P= & \left(P_{0}, 0\right) .
\end{aligned}\right.
$$

$$
h=\chi \lambda^{2} \quad(0<\chi \leqq 1 ; \lambda, \text { sufficiently small }) .
$$

Then the solution of $(3.86)^{\prime}$ is given by

$$
u=\sum_{v=0}^{\infty} u^{(\nu)}, u^{(\nu)}=P u^{(\nu-1)} .
$$


The convergence and the estimates of (3.89) will be found in the next section almost along the line of [14].

\section{$\S$ 4. Estimates for Green Matrix}

4. 1. Lemmas of Integral Operators. Let us introduce the notations and some function classes.

$$
\begin{aligned}
& Q_{\tau, \tau_{1}}=\Omega \times\left(\tau, \tau_{1}\right], Q_{\tau, \tau_{1}}^{(k)}=\Omega^{(k)} \times\left(\tau, \tau_{1}\right], \Gamma_{\tau, \tau_{1}}=\Gamma \times\left[\tau, \tau_{1}\right], \\
& d(x, t ; y, \tau)=|x-y|+|t-\tau|^{1 / 2}, d(\xi, \Gamma)=\inf _{\eta \in \Gamma} d(\xi, \eta), \\
& \Psi_{d}(x, t ; \xi, \tau)=\exp \left[-d \frac{|x-\xi|^{2}}{t-\tau}\right], \\
& \rho_{d}(t, \tau ; \xi)=\exp \left[-d \frac{d^{2}(\xi, \Gamma)}{t-\tau}\right], \\
& \Phi_{d}(x, t ; \xi, \tau)=\Psi_{d}(x, t ; \xi, \tau) \rho_{d}(t, \tau ; \xi) .
\end{aligned}
$$

(i) $U_{C, d}^{k+\alpha, m, n}\left(Q_{\tau, \tau_{1}}, \bar{Q}_{T}\right)=\left\{u(x, t ; \xi, \tau) \mid(x, t) \in Q_{\tau, \tau_{1}}, \quad(\xi, \tau) \in \bar{Q}_{T}\right.$,

$$
\begin{aligned}
& \left|D_{t}^{r} D_{x}^{s} u\right| \leqq C d^{-3-2 r-i s \mid-m-n}(x, t ; \xi, \tau)(t-\tau)^{n / 2} \\
& \quad \times \Psi_{d}(x, t ; \xi, \tau),(2 r+|s| \leqq k), \\
& \left|\Delta_{x}^{x_{0}} D_{t}^{r} D_{x}^{s} u\right| \leqq C\left|x-x_{0}\right|^{\alpha} d^{-3-2 r-|s|-\alpha}\left(x^{\prime \prime}, t ; \xi, \tau\right)(t-\tau)^{n / 2} \\
& \quad \times \Psi_{d}\left(x^{\prime \prime}, t ; \xi, \tau\right), \\
& \quad\left(2 r+|s| \leqq k,\left|x^{\prime \prime}-\xi\right|=\min \left(|x-\xi|,\left|x_{0}-\xi\right|\right)\right), \\
& \left|\Delta_{t}^{t_{0}} D_{t}^{r} D_{x}^{s} u\right|_{\mid} \leqq C\left(t-t_{0}\right)^{(k-2 r-s i+\alpha) / 2} d^{-3-2 r-|s|-m-n-\alpha} \\
& \quad \times\left(x, t_{0} ; \xi, \tau\right)(t-\tau)^{n / 2} \Psi_{d}(x, t ; \xi, \tau), \\
& \left.\quad\left(k-2<2 r+|s| \leqq k, t>t_{0}>\tau\right)\right\} .
\end{aligned}
$$

(ii) $H_{d}{ }^{k+\alpha, m}\left(\bar{Q}_{\tau, \tau_{1}}, \bar{Q}_{T}\right)\left[\right.$ resp. $\left.H_{d}{ }^{k+\alpha, m}\left(\Gamma_{\tau, \tau_{1}}, \bar{Q}_{T}\right)\right]=\{h(x, t ; \xi, \tau) \mid$

$$
(x, t) \in \bar{Q}_{\tau, \tau_{1}}\left[\operatorname{resp} . \quad(x, \iota) \in \Gamma_{r, \tau_{1}}\right], \quad(\xi, \tau) \in \bar{Q}_{T},
$$

$$
\begin{aligned}
& \left|D_{t}^{r} D_{x}^{s} h\right| \leqq C(t-\tau)^{(k-2 r-|s|+\alpha) / 2} d^{-3-m-k-\alpha}(x, t ; \xi, \tau) \\
& \quad \times \Phi_{d}(x, t ; \xi, \tau),(2 r+|s| \leqq k), \\
& \left|\Delta_{x}^{x_{0}} D_{t}^{r} D_{x}^{s} h\right| \leqq C\left|x-x_{0}\right|^{\alpha}(t-\tau)^{(k-2 r-|s| /) 2} \\
& \quad \times d^{-3-m-k-\alpha}\left(x^{\prime \prime}, t ; \xi, \tau\right) \Phi_{d}\left(x^{\prime \prime}, t ; \xi, \tau\right) \quad(2 r+|s| \leqq k),
\end{aligned}
$$




$$
\begin{gathered}
\left|\Delta_{t}^{t_{0}} D_{t}^{r} D_{x}^{s} h\right| \leqq C\left(t-t_{0}\right)^{(k-2 r-|s|+\alpha) / 2} d^{-3-m-k-\alpha}\left(x, t_{0} ; \xi, \tau\right) \\
\left.\times \Phi_{d}(x, t ; \xi, \tau) \quad(k-2<2 r+|s| \leqq k)\right\} .
\end{gathered}
$$

When $x \in \Gamma, D_{x}^{s}$ means $D_{\bar{x}^{\prime}}^{s}$. In $H_{d}{ }^{k+\alpha, 2}\left(\bar{Q}_{\tau, \tau_{1}}, \bar{Q}_{T}\right)$ and $H_{d}{ }^{k+\alpha, 1}\left(\Gamma_{\tau, \tau_{1}}\right.$, $\bar{Q}_{T}$ ) we add to the following conditions, respectively,

$$
\left|I^{(k)}(h)\right|=\left|\int_{Q_{1}(k)} \zeta^{(k)}(y) h\left(y, \tau_{0} ; \xi, \tau\right) d y d \tau_{0}\right| \leqq C \rho_{d}(t, \tau ; \xi)
$$

for any $k$,

$$
\left|J^{\left(k^{\prime \prime}\right)}(h)\right|=\left|\iint_{K_{1}^{\prime}} \bar{\zeta}^{\left(k^{\prime \prime}\right)}\left(\bar{y}^{\prime}\right) \bar{h}\left(\bar{y}^{\prime}, \tau_{0} ; \hat{\xi}, \tau\right) d \bar{y}^{\prime} d \tau_{0}\right| \leqq C \rho_{d}(t, \tau ; \xi)
$$

for any $k^{\prime \prime}$, where $Q_{1}{ }^{(k)}=\bar{Q}_{\tau, t}^{(k)} \cap\left\{d\left(y, \tau_{0} ; \xi, \tau\right) \leqq \frac{1}{2}(t-\tau)^{1 / 2}\right\}, \quad K_{1}{ }^{\prime}=K^{\prime}$ $\times[\tau, t] \cap\left\{\left(\bar{y}^{\prime}, \tau_{0}\right) \mid d\left(\bar{y}^{\prime}, F\left(\bar{y}^{\prime}\right), \tau_{0} ; \hat{\xi}, \tau\right) \leqq \frac{1}{2}(t-\tau)^{1 / 2}\right\}$.

(iii) $\stackrel{\circ}{H}_{d}{ }^{k+\alpha}\left(\bar{Q}_{\tau_{1}, \tau_{2}}, \bar{Q}_{T}\right)\left[\right.$ resp. $\left.\stackrel{\circ}{t}^{k+\alpha}\left(\Gamma_{\tau_{1}, \tau_{2}}, \bar{Q}_{T}\right)\right]=\{h(x, t ; \xi, \tau) \mid$

$$
(x, t) \in \bar{Q}_{\tau_{1}, \tau_{2}}\left[\operatorname{resp} .(x, t) \in \Gamma_{\tau_{1}, \tau_{2}}\right],(\xi, \tau) \in \bar{Q}_{T}\left(\tau_{1}>\tau\right),
$$

$$
\begin{gathered}
\left|D_{t}^{r} D_{x}^{s} h\right| \leqq C\left(t-\tau_{1}\right)^{(k-2 r-|s|+\alpha) / 2} \Phi_{d}(x, t ; \xi, \tau) \quad(2 r+|s| \leqq k), \\
\left|\Delta_{x}^{x_{0}} D_{t}^{r} D_{x}^{s} h\right| \leqq C\left|x-x_{0}\right|^{\alpha}\left(t-\tau_{1}\right)^{(k-2 r-|s|) / 2} \Phi_{d}\left(x^{\prime \prime}, t ; \xi, \tau\right) \\
(2 r+|s| \leqq k),
\end{gathered}
$$

$$
\begin{gathered}
\left|\Delta_{t}^{t_{0}} D_{t}^{r} D_{x}^{s} h\right| \leqq C\left(t-t_{0}\right)^{(k-2 r-|s|+\alpha) / 2} \Phi_{d}(x, t ; \xi, \tau) \\
\left.\left(k-2<2 r+|s| \leqq k, \quad \tau_{2}>t \geqq t_{0}>\tau_{1}\right)\right\} . \\
\hat{H}_{d}{ }^{k+\alpha}\left(\bar{Q}_{\tau_{1}, \tau_{2}}, \bar{Q}_{T}\right)\left[\operatorname{resp} . \hat{H}_{d}{ }^{k+\alpha}\left(\Gamma_{\tau_{1}, \tau_{2}}, \bar{Q}_{T}\right)\right] \\
=\left\{h(x, t ; \xi, \tau) \mid(x, t) \in \bar{Q}_{\tau_{1}, \tau_{2}}\left[\operatorname{resp} .(x, t) \in \Gamma_{\tau_{1}, \tau_{2}}\right],\right. \\
(\xi, \tau) \in \bar{Q}_{T},\left(\tau_{1}>\tau\right),
\end{gathered}
$$

(4. 12) $\left|D_{t}^{r} D_{x}^{s} h\right| \leqq C \Phi_{d}(x, t ; \xi, \tau) \quad(2 r+|s| \leqq k)$,

(4.13) $\left|\Delta_{x}{ }^{x_{0}} D_{t}{ }^{r} D_{x}{ }^{s} h\right| \leqq C\left|x-x_{0}\right|^{\alpha} \Phi_{d}\left(x^{\prime \prime}, t ; \xi, \tau\right) \quad(2 r+|s| \leqq k)$,

(4. 14) $\quad\left|\Delta_{t}^{t_{0}} D_{t}{ }^{r} D_{x}{ }^{s} h\right| \leqq C\left(t-t_{0}\right)^{(k-2 r-|s|+\alpha) / 2} \Phi_{d}(x, t ; \xi, \tau)$

$$
(k-2<2 r+|s| \leqq k)\} .
$$

We define the norm $\|h\|$ of a function $h$ in (ii) and (iii) by the minimum constant $C$ in (4.4)-(4.14). 
(iv) $\quad \dot{C}^{k+\alpha}\left(\bar{Q}_{\tau, \tau_{1}}\right)\left[\operatorname{resp} . \stackrel{\circ}{C}^{k+\alpha}\left(\Gamma_{\tau, \tau_{1}}\right)\right]=\left\{f(x, t) \in C_{x, t}^{k+\alpha,(k+\alpha) / 2}\left(\bar{Q}_{\tau, \tau_{1}}\right)[\right.$ resp.

$$
\begin{gathered}
\left.\left.f(x, t) \in C_{x, t}^{k+a,(k+\alpha) / 2}\left(\Gamma_{\tau, \tau_{1}}\right)\right]\left|D_{t}^{r} f(x, t)\right|_{t=\tau}=0\left(r=0,1, \cdots,\left[\frac{k}{2}\right]\right)\right\}, \\
C_{0}{ }^{k+\alpha}\left(\bar{Q}_{\tau, \tau_{1}}\right)=\left\{f \in C_{x, t}^{k+\alpha,(k+\alpha) / 2}\left(\bar{Q}_{\tau, \tau_{1}}\right)\left|D_{t}^{r} f\right|_{t=\tau, x \in \Gamma}=0\right. \\
\left.\left(r=0,1, \cdots,\left[\frac{k}{2}\right]\right)\right\},
\end{gathered}
$$

where

$$
\begin{aligned}
& C_{x, t}^{k+a,(k+\alpha) / 2}\left(\bar{Q}_{T}\right)=\left\{\left.f\left|\|f\| \|_{T^{(k+\alpha)}} \equiv \sum_{2 r+|s|=0}^{k}\right| D_{t}^{r} D_{x}^{s} f\right|_{T}{ }^{(0)}\right. \\
& \left.+\sum_{2 r+|s|=k}\left|D_{t}^{r} D_{x}^{s} f\right|_{x, T}^{(\alpha)}+\sum_{2 r+|s|=\max (k-1,0)}^{k}\left|D_{t}^{r} D_{x}^{s} f\right|_{t, T}^{((k-2 r-|s|+a) / 2)}<+\infty\right\}, \\
& C_{x, t}^{k+\alpha,(k+\alpha) / 2}\left(\Gamma_{T}\right)=\left\{f \mid\|f\|\left\|_{r_{T}}^{(k+\alpha)} \equiv \sup _{j}\right\| f^{(j)} \|_{\tilde{\Gamma}_{T}(j)}^{(k+\alpha)}<+\infty \quad(\mathrm{cf} . \text { (1. 19)) }\} .\right.
\end{aligned}
$$

At first we give some fromulas.

Lemma 4.1. If $\lambda$ be small, then for $x, y \in \bar{\Omega}$

$$
|\bar{x}-\bar{y}|^{2} \geqq \frac{1}{2}|\widehat{x}-\widehat{y}|^{2}=\frac{1}{2}|x-y|^{2} .
$$

\section{Corollary of Lemma 4.1.}

$$
\begin{gathered}
\Psi_{d}\left(x, t ; y, \tau_{0}\right) \Psi_{d}\left(y, \tau_{0} ; \xi, \tau\right) \leqq \Psi_{d}(x, t ; \hat{\xi}, \tau) \\
\left(\tau<\tau_{0}<t ; x, y, \xi \in \bar{\Omega}\right), \\
\left\{\begin{array}{c}
d(\bar{x}, t ; \bar{y}, \tau) \geqq \frac{1}{\sqrt{2}} d(\widehat{x}, t ; \widehat{\xi}, \tau)=\frac{1}{\sqrt{2}} d(x, t ; \xi, \tau), \\
\Phi_{d}\left(\bar{x}, t ; \bar{y}, \tau_{0}\right) \leqq \Phi_{(1 / 2) d}\left(\widehat{x}, t ; \hat{y}, \tau_{0}\right)=\Phi_{(1 / 2) d}\left(x, t ; y, \tau_{0}\right),
\end{array}\right.
\end{gathered}
$$

for $\bar{x}, \bar{y} \in K$ rith sufficient small $\lambda$.

Lemma 4. 2. For any $k>0$

$$
(t-\tau)^{-k / 2} \Psi_{d}(x, t ; \xi, \tau) \leqq e^{d} K\left(\frac{k}{2}, \frac{1}{2}\right) d^{-k}(x, t ; \xi, \tau) \Psi_{(1 / 2) d}(x, t ; \xi, \tau) .
$$

It is easily seen that Lemmas $3.3,3.15,4.1$ and 4.2 imply that

$$
\begin{aligned}
& Z_{0} \in U_{C_{28}, d_{1}}^{k_{0}+\alpha_{0}, 0, n}\left(R^{3} \times(\tau, T], R^{3} \times[0, T)\right), \\
& H_{1}{ }^{\left(k^{\prime \prime}\right)} \in U_{C_{2 \eta n}, d_{2}}^{k_{0}+\alpha_{0}, 1, n}\left(R_{+}{ }^{3} \times(\tau, T], R^{3} \times[0, T)\right),
\end{aligned}
$$


with any integer $k_{0} \geqq 0, \forall \alpha_{0} \in(0,1], \forall n \geqq 0, \forall T>0 \quad\left(R_{+}{ }^{3} \equiv\left\{\bar{x}_{3} \geqq 0\right\}\right)$.

According to the notations in (3.42) we consider the following operators :

$$
\begin{gathered}
(4.20) \quad R^{(k)} h=\int_{\tau}^{t} d \tau_{0} \int_{\Omega(k)} R^{(k)}\left(x, t ; y, \tau_{0}\right) \zeta^{(k)}(y) h\left(y, \tau_{0} ; \xi, \tau\right) d y, \\
(4.21) \quad R_{1} h=\Pi_{\bar{x}, \hat{\xi}}^{x, \xi} \bar{R}_{1} h=\prod_{\bar{x}, \xi}^{x, \xi} \int_{\tau}^{t} d \tau_{0} \int_{K^{\prime}} H_{1}^{\left(k^{\prime \prime}\right)}\left(\bar{x}-\bar{y}^{\prime}, t-\tau_{0}\right) \bar{\zeta}^{\left(k^{\prime \prime}\right)}\left(\bar{y}^{\prime}\right) \\
\times \bar{h}\left(\bar{y}^{\prime}, \tau_{0} ; \hat{\xi}, \tau\right) d \bar{y}^{\prime},
\end{gathered}
$$

where

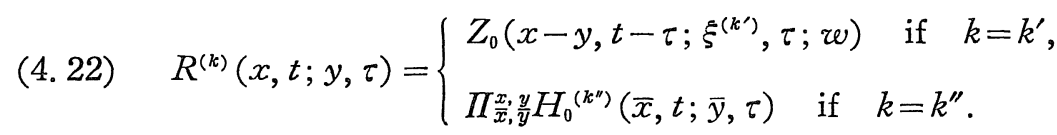

In order to estimate $H_{0}^{\left(k^{n}\right)}$ we begin with the following lemma.

\section{Lemma 4.3. Suppose}

$$
\Gamma \in C^{2+k+\alpha}, \tau_{1}-\tau=\chi \lambda^{2},
$$

where $\chi \leqq 1$ and $\lambda$ is sufficiently small so as to satisfy (4.15). Then $v_{1}=R_{1} h \in H_{d^{\prime}}^{k+\alpha, 0}\left(\bar{Q}_{\tau, \tau_{1}}^{\left(k^{\prime \prime}\right)}, \bar{Q}_{T}\right)$ and $\left\|v_{1}\right\| \leqq C_{28}\|h\|\left(d^{\prime}=\min \left(d, \frac{1}{2} d_{2}\right)\right)$ provided $h \in H_{d}{ }^{k+\alpha, 0}\left(\Gamma_{\tau, \tau_{1}}, \bar{Q}_{T}\right)$.

Proof. It is sufficient to estimate $D_{\bar{x}}^{s} \bar{v}_{1}=D_{\bar{x}}^{s} \bar{R}_{1} h(\bar{x} \in K)$. Let us make use of the following notations:

$$
\begin{aligned}
& (x, t)=P, \quad\left(y, \tau_{0}\right)=Q, \quad(\xi, \tau)=M, \\
& (\bar{x}, t)=\bar{P}, \quad\left(\bar{y}, \tau_{0}\right)=\bar{Q}, \quad(\hat{\xi}, \tau)=\widehat{M}, \\
& \left(\bar{x}^{\prime}, t\right)=\bar{P}^{\prime}, \quad\left(\bar{y}^{\prime}, \tau_{0}\right)=\bar{Q}^{\prime}, \quad\left(\bar{\xi}^{\prime}, \tau\right)=\bar{M}^{\prime}, \\
& d y d \tau_{0}=d Q, d \bar{y}^{\prime} d \tau_{0}=d \bar{Q}^{\prime}, \\
& d_{0}=d(P, M)=|x-\xi|+(t-\tau)^{1 / 2} .
\end{aligned}
$$

Let the integral domain $K^{\prime} \times[\tau, t]$ be divided into three parts $K_{i}^{\prime}$ $(i=1,2,3)$ such that $K^{\prime} \times[\tau, t]=K_{1}^{\prime} \cup K_{2}^{\prime} \cup K_{3}^{\prime}$, where

$$
K_{1}^{\prime}=K^{\prime} \times[\tau, t] \cap\left\{\left(\bar{y}^{\prime}, \tau_{0}\right) \mid d\left(\bar{y}^{\prime}, F\left(\bar{y}^{\prime}\right), \tau_{0} ; \widehat{M}\right)\right.
$$




$$
\begin{gathered}
\left.=d(Q, M) \leqq \frac{1}{2}(t-\tau)^{1 / 2}\right\}, \\
K_{2}^{\prime}=K^{\prime} \times[\tau, t] \cap\left\{\left(\bar{y}^{\prime}, \tau_{0}\right) \mid d\left(\bar{x}^{\prime}, \bar{x}_{3}+F\left(\bar{x}^{\prime}\right), t ; \bar{y}^{\prime}, F\left(\bar{y}^{\prime}\right), \tau_{0}\right)\right. \\
\left.=d(P, Q) \leqq \frac{1}{2}(t-\tau)^{1 / 2}\right\}, \\
K_{3}^{\prime}=K^{\prime} \times[\tau, t]-K_{1}^{\prime}-K_{2}^{\prime} .
\end{gathered}
$$

Let $\bar{x} \in K$ and $\bar{x}_{3}>0$.

$$
\begin{aligned}
D_{\bar{x}^{s}} \bar{v}_{1}(\bar{P}, \widehat{M}) & =\iint_{K_{1^{\prime}}}\left[D_{\bar{x}}{ }^{s} H_{1}{ }^{\left(k^{\prime \prime}\right)}\left(\bar{P}-\bar{Q}^{\prime}\right)-D_{\bar{x}}{ }^{s} H_{1}{ }^{\left(k^{\prime \prime}\right)}\left(\bar{P}^{\prime \prime}-\bar{M}^{\prime}\right)\right] \\
& \times \bar{\zeta}^{\left(k^{\prime \prime}\right)}\left(\bar{y}^{\prime}\right) \bar{h}\left(\bar{Q}^{\prime}, \widehat{M}\right) d \bar{Q}^{\prime} \\
& +D_{\bar{x}}^{s} H_{1}{ }^{\left(k^{\prime \prime}\right)}\left(\bar{P}^{\prime \prime}-\overline{M^{\prime}}\right) \iint_{K_{1^{\prime}}} \bar{\zeta}^{\left(k^{\prime \prime}\right)}\left(\bar{y}^{\prime}\right) \bar{h}\left(\bar{Q}^{\prime}, \widehat{M}\right) d \bar{Q}^{\prime} \\
& +\iint_{K_{2^{\prime}}} D_{\bar{x}}{ }^{s} H_{1}{ }^{\left(k^{\prime \prime}\right)}\left(\bar{P}-\bar{Q}^{\prime}\right) \bar{\zeta}^{\left(k^{\prime \prime}\right)}\left(\bar{y}^{\prime}\right) \bar{h}\left(\bar{Q}^{\prime}, \widehat{M}\right) d \bar{Q}^{\prime} \\
& +\int_{K_{\mathbf{s}^{\prime}}} \int_{\bar{x}^{s}} H_{1}{ }^{\left(k^{\prime \prime}\right)}\left(\bar{P}-\bar{Q}^{\prime}\right) \bar{\zeta}^{\left(k^{\prime \prime}\right)}\left(\bar{y}^{\prime}\right) \bar{h}\left(\bar{Q}^{\prime}, \widehat{M}\right) d \bar{Q}^{\prime} \\
& \equiv \sum_{i=1}^{4} L_{1}{ }^{(i)},
\end{aligned}
$$

where $\bar{P}^{\prime \prime}=\left(\bar{x}^{\prime},\left|\bar{x}_{3}-\bar{\xi}_{3}\right|, t\right)$ and $|s| \leqq k$. Moreover it is sufficient to consider the case $\xi \in \widetilde{\Omega}^{\left(k^{\prime \prime}\right)}\left(\widetilde{\Omega}^{\left(k^{\prime \prime}\right)}\right.$ is a neighborhood of $\Omega^{\left(k^{\prime \prime}\right)}$ contained in a Lyapunov sphere with $\xi^{\left(k^{\prime \prime}\right)} \in \Gamma$ its center). Indeed if $\xi \notin \widetilde{\Omega}^{\left(k^{\prime \prime}\right)}$, then $K_{1}^{\prime}=\varnothing$ for sufficiently small $\chi$ because $t-\tau \leqq \tau_{1}-\tau \leqq \chi \lambda^{2}$.

$L_{1}^{(i)}(i=1,2)$ can easily be estimated, i.e.,

(4. 25) $\left|L_{1}{ }^{(i)}\right| \leqq C_{28}^{(i)}\|h\|(t-\tau)^{(k-|s|+\alpha) / 2} d_{0}^{-3-k-\alpha} \Phi_{d^{\prime}}(P, M) \quad(i=1,2)$.

$$
\begin{aligned}
& \left|L_{1}^{(4)}\right| \leqq C_{28}^{(4)}\|h\|(t-\tau)^{(2 k-|s|+2 \alpha) / 2} \bar{\Phi}_{d^{\prime}}(P, M) \\
& \quad \times \iint_{K_{\mathbf{s}^{\prime}}} d^{-4-k-\alpha}(P, Q) d^{-3-k-\alpha}(Q, M) d \bar{Q}^{\prime} . \\
& \iint_{K_{\mathbf{s}^{\prime}}} d^{-4-k-\alpha}(P, Q) d^{-3-k-\alpha}(Q, M) d \bar{Q}^{\prime} \\
& \quad=\int_{K_{\mathbf{s}^{\prime}} \cap\left\{d(P, Q) \leq(1 / 2) d_{0}\right\}}[\cdots] d \bar{Q}^{\prime}+\int_{K_{\mathbf{s}^{\prime}} \cap\left\{d(P, Q)>(1 / 2) d_{0}\right\}}[\cdots] d \bar{Q}^{\prime}
\end{aligned}
$$




$$
\begin{aligned}
& \equiv L_{1}{ }^{(4,1)}+L_{1}{ }^{(4,2)} . \\
& L_{1}{ }^{(4,1)} \leqq C_{28}^{(4,1)} d_{0}{ }^{-3-k-\alpha}(t-\tau)^{-(k+\alpha) / 2} \text {, } \\
& L_{1}^{(4,2)} \leqq\left\{\begin{array}{c}
C_{28}^{(4,2,1)} d_{0}{ }^{-4-k-\alpha}\left\{(t-\tau)^{(1-k-2 \alpha) / 2}\right. \\
\times \int_{d\left(\bar{Q}^{\prime}, \bar{M}^{\prime}\right) \leq(1 / 2)(t-\tau)^{1 / 2}} d^{-4+\alpha}\left(\bar{Q}^{\prime}, \bar{M}^{\prime}\right) d \bar{Q}^{\prime} \\
\left.+\int_{d\left(\bar{Q}^{\prime}, \bar{M}^{\prime}\right)>(1 / 2)(t-\tau)^{1 / 2}} d^{-3-k-\alpha}\left(\bar{Q}^{\prime}, \bar{M}^{\prime}\right) d \bar{Q}^{\prime}\right\}, \\
\quad \text { if } 1-k-\alpha \leqq 0 ; \\
C_{28}^{(4,2,2)} d_{0}^{-4-k-\alpha} \iint_{d\left(\bar{Q}^{\prime}, \bar{M}^{\prime}\right) \leqq 2 d_{0}} d^{-3-k-\alpha}\left(\bar{Q}^{\prime}, \bar{M}^{\prime}\right) d \bar{Q}^{\prime} \\
\quad \int_{d\left(\bar{Q}^{\prime}, \bar{M}^{\prime}\right)>2 d_{0}} d^{-4-k-\alpha}\left(\bar{P}^{\prime}, \bar{Q}^{\prime}\right) d^{-3-k-\alpha}\left(\bar{Q}^{\prime}, \bar{M}^{\prime}\right) d \bar{Q}^{\prime}, \\
\quad \text { if } 1-k-\alpha>0,
\end{array}\right. \\
& \leqq C_{28}^{(4,2)} d_{0}^{-3-k-\alpha}(t-\tau)^{-(k+\alpha) / 2} \text {. }
\end{aligned}
$$

Therefore we have

$$
\left|L_{1}^{(4,2)}\right| \leqq C_{28}^{(4)}\left(C_{28}^{(4,1)}+C_{28}^{(4,2)}\right)\|h\|(t-\tau)^{(k-|s|+\alpha) / 2} d_{0}^{-3-k-\alpha} \Phi_{d^{\prime}}(P, M) .
$$

In the next place as concerns $L_{1}{ }^{(3)}$ we make use of the formulas (3.67) and (3.70). If we put

$$
\hat{\mathfrak{A}}^{\left(k^{\prime \prime}\right)}\left(\xi^{\left(k^{\prime \prime}\right)}, \tau, w ; D_{\bar{x}}, D_{t}\right) K^{(v)}(\bar{x}, t)=\widetilde{K}^{(v)}(\bar{x}, t),
$$

then from Lemma 3.14 it easily follows that

$$
\begin{aligned}
H_{1}{ }^{\left(k^{\prime \prime}\right)}(\bar{x}, t) & =\left[D_{t}-\left(1+\delta_{3}\right) \Delta_{(2)}\right]^{v} \widetilde{K}^{(v)}(\bar{x}, t) \\
& =\sum_{2 \mu_{0}+\mid \mu^{\prime} j=2 v} a_{\mu_{0}, \mu^{\prime}} D_{t}^{\mu_{0}{ }^{\prime}} D_{\bar{x}^{\prime}}^{\mu^{\prime}} \widetilde{K}^{(v)}(\bar{x}, t),
\end{aligned}
$$

(4. 29) $\quad \widetilde{K}^{(v)}\left(\bar{P}-\bar{Q}^{\prime}\right) \in U_{C_{20}, d_{2}}^{k_{0}+\alpha_{0}, 2(1-v)-1, n}\left(R^{3} \times\left(\tau_{0}, T\right], R^{3} \times[0, T)\right)$

$$
\left(\forall k_{0}=0,1,2, \cdots ; \forall \alpha_{0} \in(0,1] ; \forall n \geqq 0 ; \forall T>0\right) \text {. }
$$

Now we assume that in (4.28) $v=\left[\frac{|s|}{2}\right]+1$. Let $m_{i} \leqq \mu_{i}(i=0,1,2)$ hold and let $2 m_{0}+\left|m^{\prime}\right|$ take a maximum value not exceeding $|s|$, i.e., $2 m_{0}+\left|m^{\prime}\right|=2\left[\frac{|s|}{2}\right]$ or $|s|$. Integrations by parts imply that 
(4. 30) $L_{1}^{\left({ }^{3}\right)}=\sum_{\Omega \mu_{0}+\left|\mu^{\prime}\right|=2 v} a_{\mu_{0}, \mu^{\prime}}\left\{\iint_{K_{2}} D_{t}^{\mu_{0}^{\prime}-m_{0}} D_{\bar{X}^{\prime},-m^{\prime}} D_{\bar{x}}^{s} \widetilde{K}^{(v)}\left(\bar{P}-\bar{Q}^{\prime}\right)\right.$

$$
\begin{aligned}
& \times D_{\tau_{0}}^{m_{0}} D_{\bar{y}^{\prime}}^{m^{\prime}}\left[\bar{\zeta}^{\left(k^{\prime \prime}\right)}\left(\bar{y}^{\prime}\right) \bar{h}\left(\bar{Q}^{\prime}, M\right)\right] d \bar{Q}^{\prime}
\end{aligned}
$$

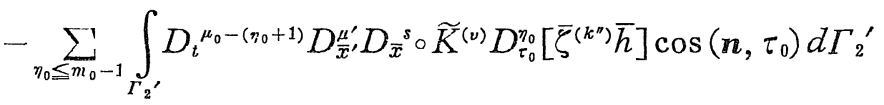

$$
\begin{aligned}
& \left.-\sum_{\left|\eta^{\prime}\right| \leqq\left|m^{\prime}\right|-1} \int_{\Gamma_{2}^{\prime}} D_{t}^{\mu_{0}-m_{0}} D_{\overline{\bar{x}}^{\prime}-\left(\eta^{\prime}+1\right)}^{\mu^{\prime}} D_{\bar{x}^{s}}^{s} \widetilde{K}^{(v)} D_{\tau_{0}}^{m_{0}} D_{\bar{y}^{\prime}}^{\eta^{\prime}}\left[\bar{\zeta}^{\left(k^{\prime \prime}\right)} \bar{h}\right] \cos \left(\boldsymbol{n}, \bar{y}^{\prime}\right) d \Gamma_{2}^{\prime}\right\} \\
& =\sum_{2 \mu_{0}+\left\lceil\mu^{\prime} \mid=2 v\right.} a_{\mu_{0}, \mu^{\prime}}\left(L_{1}^{(3,1)}+L_{1}^{(3,2)}+L_{1}^{(3,3)}\right),
\end{aligned}
$$

where $\Gamma_{2}^{\prime}$ is a boundary of $K_{2}^{\prime}$ and $\boldsymbol{n}$ is an inner normal vector at each point of $\Gamma_{2}^{\prime} . \quad L_{1}^{(3, i)}(i=2,3)$ can easily be estimated as follows:

$$
\left|L_{1}^{(3, i)}\right| \leqq C_{28}^{(3, i)}\|h\|(t-\tau)^{(k--|s|+\alpha) / 2} d_{0}^{-3-k-\alpha} \Phi_{d^{\prime}}(P, M) \quad(i=2,3) .
$$

In order to estimate $L_{1}^{(3,1)}$, let $t_{0}=\min \left\{t \mid\left(\bar{x}^{\prime}, t\right) \in K_{2}^{\prime}\right\}, t_{i}=t-2^{-i}\left(t-t_{0}\right)$, $K_{2 i}^{\prime}=K_{2}^{\prime} \cap\left(t_{i}, t_{i+1}\right)$. Then

$$
\begin{aligned}
& L_{1}^{(3,1)}=\sum_{i=0}^{\infty} \int_{K^{\prime}} \int_{2 i} D_{t}^{\mu_{0}-m_{0}} D_{\bar{x}^{\prime}-m^{\prime}}^{\mu^{\prime}} D_{\bar{x}^{s}} \widetilde{K}^{(v)} D_{\tau_{0}}^{m_{0}} D_{\bar{y}^{\prime}}^{m^{\prime}}\left[\bar{\zeta}^{\left(k^{\prime \prime}\right)} \bar{h}\right] d \bar{Q}^{\prime} \\
& =\sum_{i=0}^{\infty} \sum_{\left|\eta^{\prime}\right| \leqq m^{\prime} !} c_{m^{\prime}, \eta^{\prime}} \int_{K^{\prime}} \int_{2 i} D_{t}^{\mu_{0}-m_{0}} D_{\bar{x}^{\prime}}^{\mu^{\prime}-m^{\prime}} D_{\bar{x}}^{s} \widetilde{K}^{(v)} D_{\frac{y^{\prime}}{\eta^{\prime}}, \bar{\zeta}^{\left(k^{\prime \prime}\right)}} \\
& \times \Delta_{\tau_{0}}^{t_{i}} D_{\tau_{0}}^{m_{0}} D_{\bar{y}^{\prime}}^{m^{\prime}-\eta^{\prime}} \bar{h} d \bar{Q}^{\prime}+\sum_{i=0}^{\infty} \sum_{\left|\eta^{\prime}\right| \leq\left|m^{\prime}\right|} c_{m^{\prime}, \eta^{\prime}} \iint_{K^{\prime}} \int_{2 i} D_{t}^{\mu_{0}-m_{0}} D_{\bar{x}^{\prime}}^{\mu^{\prime}, m^{\prime}} \\
& \cdot D_{\bar{x}}^{s} \widetilde{K}^{(v)} D_{\bar{y}}^{\eta^{\prime}} \bar{\zeta}^{\left(k^{\prime \prime}\right)} D_{t_{i}}^{m_{0}} D_{\bar{y}^{\prime}}^{m^{\prime}-\eta^{\prime}} \bar{h} d \bar{Q}^{\prime} \equiv L_{1}{ }^{(3,1,1)}+L_{1}{ }^{(3,1,2)} .
\end{aligned}
$$

$$
\left|L_{1}^{(3,1,1)}\right| \leqq C_{28}^{(3,1,1)}\|h\|(t-\tau)^{(k-|s|+\alpha) / 2} d_{0}^{-3-k-\alpha} \Phi_{d^{\prime}}(P, M) .
$$

Next we transform $L_{1}^{(3,1,2)}$ into the form

$$
\begin{aligned}
& L_{1}^{(3,1,2)}=\sum_{\left|\eta^{\prime}\right| \leq \leq\left|m^{\prime}\right|} c_{m^{\prime}, \eta^{\prime}} \sum_{i=0}^{\infty}\left[\int_{\bar{K}^{\prime} 2 i} \int_{t} D_{t}^{\mu_{0}-m_{0}} D_{\bar{x}^{\prime}}^{\mu^{\prime}-m^{\prime}} D_{\bar{x}}^{s} \widetilde{K}^{(v)} D_{\bar{y}}^{\eta^{\prime}} \bar{\zeta}^{\left(k^{\prime \prime}\right)}\right. \\
& \times \Delta_{\bar{y} y^{\prime}}^{\bar{z}^{\prime}} D_{t_{i}}^{m_{0}} D_{\bar{y}^{\prime}}^{m^{\prime}-\eta^{\prime}} \bar{h} d \bar{Q}^{\prime}+D_{t_{i}}^{m_{0}} D_{\bar{z}^{\prime}}^{m^{\prime}-\eta^{\prime}} \bar{h}\left\{\iint_{K^{\prime}}{ }_{2 i} D_{t}^{\mu_{0}-m_{0}} D_{\bar{x}^{\prime}}^{\mu^{\prime}-m^{\prime}-1}\right. \\
& \cdot D_{\bar{x}}^{s} \widetilde{K}^{(v)} D_{\bar{y}}^{\eta^{\prime}}+1 \bar{\zeta}^{\left(k^{\prime \prime}\right)} d \bar{Q}^{\prime}-\int_{\Gamma^{\prime} 2 i} D_{t}^{\mu_{0}-m_{0}} D_{\bar{x}^{\prime}}^{\mu^{\prime}-m^{\prime}-1} D_{\bar{x}}^{s} \widetilde{K}^{(v)}
\end{aligned}
$$

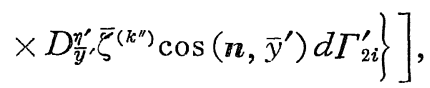


where $\left(\bar{z}^{\prime}, 0\right)$ is a $k^{\prime \prime}$-local coordinate of $z \in \Gamma \cap \Omega^{\left(k^{\prime \prime}\right)}$ which is nearest of $x$ and $\Gamma_{2 i}^{\prime}$ is a boundary of $K_{2 i}^{\prime}$, if $2 m_{0}+\left|m^{\prime}\right|=|s|$. (N.B. in this case $2 \mu_{0}+\left|\mu^{\prime}\right|-2 m_{0}-\left|m^{\prime}\right|=2\left(1-\left\{\frac{|s|}{2}\right\}\right),\left\{\frac{|s|}{2}\right\}=\frac{|s|}{2}-\left[\frac{|s|}{2}\right]$.) If $2 m_{0}+\left|m^{\prime}\right|$ $=2\left[\frac{|s|}{2}\right]$, then $2 \mu_{0}+\left|\mu^{\prime}\right|-2 m_{0}-\left|m^{\prime}\right|=2$, hence $D_{t}^{\mu_{0}-m_{0}} D_{\bar{x}^{\prime}}^{\mu^{\prime}-m^{\prime}}=D_{t}$ or $D_{\bar{x}^{\prime}}^{2}$. In the first case we have

$$
\begin{aligned}
L_{1}{ }^{(3,1,2)}= & \sum_{\left|\eta^{\prime}\right| \leq\left|m^{\prime}\right|} c_{m^{\prime}, \eta^{\prime}} \sum_{i=0}^{\infty}\left[\int_{K_{t_{i}}} D_{\bar{x}}^{s} \widetilde{K}^{(v)} D_{\bar{y}^{\prime}}^{\eta^{\prime}} \bar{\zeta}^{\left(k^{\prime \prime}\right)} \Delta_{t_{i}}^{t_{i-1}} D_{t_{i}}^{m_{0}} D_{\bar{y}^{\prime}}^{m^{\prime}-\eta^{\prime}} \bar{h} d \bar{y}^{\prime}\right. \\
& \left.-\int_{\Gamma^{\prime} 2 i} D_{\bar{x}} s \widetilde{K}^{(v)} D_{\bar{y}^{\prime}}^{\eta^{\prime}} \bar{\zeta}^{\left(k^{\prime \prime}\right)} D_{t_{i}}^{m_{0}} D_{\bar{y}^{\prime}}^{m^{\prime}-\eta^{\prime}} \bar{h} \cos \left(\boldsymbol{n}, \tau_{0}\right) d \Gamma_{2 i}^{\prime}\right],
\end{aligned}
$$

where $K_{t_{i}}=K_{2 i}^{\prime} \cap\left\{t=t_{i}\right\}$. In the second case, if we transfer $2\{q\}$ differentiation from $K^{(v)}$ to another term in (4.30) by integration by parts, then this case is reduced to the case $2 m_{0}+\left|m^{\prime}\right|=\frac{|s|}{2}$. In the sequel after some calculations we obtain

$$
\left|L_{1}^{(3,1,2)}\right| \leqq C_{28}^{(3,1,2)}\|h\|(t-\tau)^{(k-|s|+\alpha) / 2} d_{0}^{-3-k-\alpha} \Phi_{d^{\prime}}(P, M) .
$$

Hence

$$
\left|D_{\bar{x}}^{s} \bar{v}_{1}\right| \leqq C_{28,1}\|h\|(t-\tau)^{(k-|s|+\alpha) / 2} d_{0}^{-3-k-\alpha} \Phi_{d^{\prime}}(P, M),
$$

where $C_{28,1}=C_{28}^{(1)}+C_{28}^{(2)}+C_{28}^{(3,1,1)}+C_{28}^{(3,1,2)}+C_{28}^{(4)}\left(C_{28}^{(4,1)}+C_{28}^{(4,2)}\right)$.

As for $\Delta_{t}^{t_{0}} D_{\bar{x}}^{s} \bar{v}_{1}$ and $\Delta_{x}{ }^{x_{0}} D_{\bar{x}}^{s} \bar{v}_{1}$, we can obtain the desired estimates by an analogous way as above.

Q.E.D.

(4. 8) implies that

$$
\begin{gathered}
\left.Z_{0}^{\left(k^{\prime \prime}\right)}\right|_{\bar{x}_{3}=0} \in H_{d_{1}}^{k_{0}+\alpha_{0}, 0}\left(R^{2} \times(\tau, T], R_{+}{ }^{3} \times[0, T)\right) \\
\left(\forall k_{0}=0,1,2, \cdots ; \forall \alpha_{0} \in(0,1]\right) .
\end{gathered}
$$

Since $\left\{\bar{x}_{3}=0\right\} \in C^{\infty}$ and $\zeta \equiv 1$, by the same argument as in the proof of Lemma 4.3 (in this case, however, we do not need the condition $\tau_{1}-\tau$ $\left.\leqq \chi \lambda^{2}\right)$ we obtain

$$
\begin{gathered}
\int_{\tau}^{t} d \tau_{0} \int_{R^{2}} H_{1}{ }^{\left(k^{\prime \prime}\right)}\left(\bar{x}-\bar{y}_{0}{ }^{\prime}, t-\tau_{0}\right) Z_{0}{ }^{\left(k^{\prime \prime}\right)}\left(\bar{y}_{0}{ }^{\prime}-\bar{y}, \tau_{0} ; \xi^{\left(k^{\prime \prime}\right)}, \tau ; w\right) d \bar{y}_{0}{ }^{\prime} \\
\in H_{d_{3}}^{k_{0}+\alpha_{0}, 0}\left(R_{+}{ }^{3} \times(\tau, T], R_{+}{ }^{3} \times[0, T)\right) \\
\left(d_{3}=\min \left(d_{1}, d_{2} / 2\right)\right)
\end{gathered}
$$


hence

(4. 40) $\quad H_{0}{ }^{\left(k^{\prime \prime}\right)}\left(\bar{x}, t ; \bar{y}, \tau_{0}\right) \in U_{C_{30}, d_{3}}^{k_{0}+\alpha_{0}, 0, n}\left(R_{+}{ }^{3} \times\left(\tau_{0}, T\right], R_{+}{ }^{3} \times[0, T)\right)$

for $\forall k_{0}=0,1,2, \cdots ; \forall \alpha_{0} \in(0,1] ; \forall n \geq 0 ; \forall T>0$.

The following three lemmas can be proved in the almost same way as those used in Lemma 4.3.

Lemma 4. 4. Under the condition (4.23), $u_{k} \equiv R^{(k)} h \in H_{d^{\prime \prime}}^{2+m+\alpha, 0}$ $\left(\bar{Q}_{\tau, \tau_{1}}^{\left(k^{\prime \prime}\right)}, \bar{Q}_{T}\right)$ and $\left\|R^{(k)} h\right\| \leqq C_{31}\|h\|$, where $d^{\prime \prime}=\min \left(d, d_{2} / 2\right)$ provided that $h \in H_{d}{ }^{m+\alpha, 2}\left(\bar{Q}_{\tau, \tau_{1}}, \bar{Q}_{T}\right)$.

Lemma 4. 5. Under the condition (4.23) except that $\tau_{2}-\tau_{1}=\chi \lambda^{2}$, $R^{(k)} h\left[\right.$ resp. $\left.R_{1} h\right]$ defined by (4.20) [resp. (4.21)], where the integral interval with respect to $\tau_{0}$ is $\left(\tau_{1}, t\right)$ in place of $(\tau, t)$, is a bounded operator on $\stackrel{\circ}{H}_{d}{ }^{m+\alpha}\left(\bar{Q}_{\tau_{1}, \tau_{2}}, \bar{Q}_{T}\right) \quad\left[\operatorname{resp} . \stackrel{\circ}{H}_{d}^{m+\alpha}\left(\Gamma_{\tau_{1}, \tau_{2}}, \bar{Q}_{T}\right)\right]$ and $R^{(k)} h$ $\in \stackrel{H}{H}_{d^{\prime}}^{2+m+\alpha}\left(\bar{Q}_{\tau_{1}, \tau_{2}}^{(k)}, \bar{Q}_{T}\right) \quad\left[\right.$ resp. $\left.R_{1} h \in{\stackrel{H}{d^{\prime \prime}}}^{m+\alpha}\left(\bar{Q}_{\tau_{1}, \tau_{2}}^{(k,)}, \bar{Q}_{T}\right)\right]$.

Lemma 4. 6. Under the condition (4.23), $R^{(k)}\left[\right.$ resp. $\left.R_{1}\right]$ is a bounded mapping from $\stackrel{\circ}{C}^{m+\alpha}\left(\bar{Q}_{\tau, \tau_{1}}\right) \quad\left[\right.$ resp. $\left.\stackrel{\circ}{C}^{m+\alpha}\left(\Gamma_{\tau, \tau_{1}}\right)\right]$ into $\dot{C}^{2+m+\alpha}\left(\bar{Q}_{\tau, \tau_{1}}^{(k)}\right)$

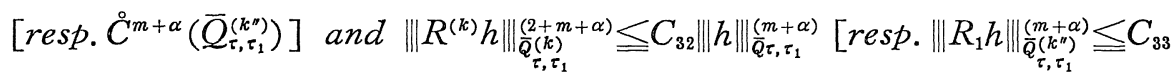
$\left.\times\|h\| \|_{\Gamma_{\tau, \tau_{1}}}^{(m+\alpha)}\right]$, where $\|\cdot\| \|_{\substack{Q(k) \\ \tau, \tau_{1}}}^{(m+\alpha)}\left[\operatorname{resp.}\|\cdot\| \|_{\Gamma_{\tau, \tau_{1}}}^{(m+\alpha)}\right]$ is defined in (iv) with $\bar{Q}_{\tau, \tau_{1}}^{(k)}$ $\left[\right.$ resp. $\left.\Gamma_{\tau, \tau_{1}}\right]$ in place of $\bar{Q}_{T}\left[\right.$ resp. $\left.\Gamma_{T}\right]$.

4. 2. Estimates for Green Matrix. As we have already seen in $\S \S 3.3$ and 3.4, Green matrix in $Q_{\tau, \tau+h}$ coincides with the unique solution of (3.86). For sufficiently small $h$, this solution is given by the series (3.89). Thus it is sufficient to estimate each term of (3.89). From Lemma 3. 6 follows that

$$
Z(x-\xi, t-\tau ; \xi, \tau ; w) \in U_{C_{34}, d_{4}}^{2+\alpha, 0, n}\left(Q_{\tau}, T, \bar{Q}_{T}\right) .
$$

Let $u^{(0)}=\left(u_{0}{ }^{(0)}, u_{1}{ }^{(0)}\right) \equiv\left(0,\left.Z\right|_{\Gamma_{r, \tau+h}}\right)$ and let $u^{(\nu)}(\nu \geqq 1)$ define by (3. 89) and $h$ satisfy $(3.88)$. Then we have

Lemma 4. 7. $u=\left(u_{0}, u_{1}\right) \in H_{d_{5}}^{\alpha, 2}\left(Q_{\tau, \tau+h}, \bar{Q}_{T}\right) \times H_{d_{5}}^{2+\alpha, 0}\left(\Gamma_{\tau, \tau+h}, \bar{Q}_{T}\right)$, where $d_{5}=d_{4} / 2$. 
Proof. By (4.41) it is clear that

$$
u_{1}{ }^{(0)} \in H_{d_{5}}^{2+\alpha, 0},\left\|u_{1}^{(0)}\right\| \leqq C_{35} .
$$

Since $P_{1}=0$, we obtain

$$
u_{1}^{(\nu)}=0 \quad \text { for } \quad \nu=1,2,3, \cdots .
$$

From (3.87), (3.89), (4.42) and Lemma 4.3 follows that

(4. 44) $\left|u_{0}^{(1)}\right| \equiv\left|P_{0} u^{(0)}\right| \leqq C_{35} C_{36}^{(1)} \Lambda(t-\tau)^{\alpha / 2} d^{-5-\alpha}(x, t ; \xi, \tau) \Phi_{d_{5}}(x, t ; \xi, \tau)$, where $\Lambda=\chi^{\alpha / 2}+\lambda^{\alpha}$. Similarly after some more calculations we have

$$
\left\{\begin{array}{c}
\left|\Delta_{x}{ }^{x_{0}} u_{0}{ }^{(1)}\right| \leqq C_{35} C_{36}^{(2)} \Lambda\left|x-x_{0}\right|^{\alpha} d^{-5-\alpha}\left(x^{\prime \prime}, t ; \xi, \tau\right) \Phi_{d_{5}}\left(x^{\prime \prime}, t ; \xi, \tau\right) \\
\left|\Delta_{t}^{t_{0}} u_{0}{ }^{(1)}\right| \leqq C_{35} C_{36}^{(3)} \Lambda\left(t-t_{0}\right)^{\alpha} d^{-5-\alpha}\left(x, t_{0} ; \xi, \tau\right) \Phi_{d_{5}}(x, t ; \xi, \tau) \\
\left(t>t_{0}>\tau\right)
\end{array}\right.
$$

Next in order to estimate $I^{(k)}\left(u_{0}{ }^{(1)}\right)$ we transform $u_{0}{ }^{(1)}$ in the form

$$
\begin{aligned}
& u_{0}^{(1)}(x, t ; \xi, \tau)=\widetilde{u}_{0}^{(1)}(x, t ; \xi, \tau) \\
& \quad+\sum_{j^{\prime \prime}} \eta^{\left(j^{\prime \prime}\right)}(x) \sum_{|s|=2}\left\{\Pi_{\bar{x}, \bar{\xi}, \hat{\xi}}^{x}\left[\widetilde{a}_{s}\left(\xi^{\left(j^{\prime \prime}\right)}, \tau ; \overline{\bar{x}}^{\prime}\right) D_{\overline{\bar{x}^{\prime}}}^{s} U_{j^{\prime \prime}}^{(0)}(\overline{\bar{x}}, t ; \hat{\xi}, \tau)\right]\right. \\
& \left.\quad+\left[a_{s}(\xi, \tau)-a_{s}\left(\xi^{\left(j^{\prime \prime}\right)}, \tau\right)\right] D_{x}^{s} U_{j^{\prime \prime}}^{(0)}(x, t ; \xi, \tau)\right\}
\end{aligned}
$$

where

$$
\left\{\begin{array}{l}
\left|\widetilde{u}_{0}^{(1)}\right| \leqq C_{35} C_{36}^{(4)}\left(1+\chi^{(1-\alpha) / 2} \lambda^{-\alpha}\right) d^{-5+\alpha}(x, t ; \xi, \tau) \Phi_{d_{5}}(x, t ; \xi, \tau) \\
\widetilde{a}_{s}\left(\xi^{\left(j^{\prime \prime}\right)}, \tau ; \overline{\bar{x}}^{\prime}\right)=\sum_{0<\left|\nu^{\prime}\right| \leqq 2} \widetilde{a}_{s \nu^{\prime}}\left(\xi^{\left(j^{\prime \prime}\right)}, \tau\right)\left(D_{\overline{x_{1}}} F\left(\overline{\bar{x}}^{\prime}\right)\right)^{\nu_{1}}\left(D_{\bar{x}_{2}} F\left(\overline{\bar{x}}^{\prime}\right)\right)^{\nu_{2}} \\
\quad\left(\nu^{\prime}=\left(\nu_{1}, \nu_{2}\right)\right) \\
\widetilde{a}_{s \nu^{\prime}}\left(\xi^{\left(j^{\prime \prime}\right)}, \tau\right) \text { and } a_{s} \text { are determined by the coefficients of }(2.18) \\
U_{j^{\prime \prime}}^{(0)}=\Pi_{\frac{x, \xi \xi}{x, \xi} \bar{U}_{j^{\prime \prime}}^{(0)}=\bar{R}_{1} u_{1}{ }^{(0)}}
\end{array}\right.
$$

and $\overline{\bar{x}}$ is an expression of $x$ by the transformation (1.17) of $j^{\prime \prime}$-local rectangular coordinate system $\hat{x}$.

From (4.46) and (4.47) we derive that

$$
\left|I^{(k)}\left(u_{0}^{(1)}\right)\right| \leqq C_{35} C_{36}^{(5)} \Lambda \rho_{5}(t, \tau ; \xi)+I,
$$

where

$$
I=\sum_{j^{\prime \prime}} \sum_{|s|=2} \mid \int_{Q_{1}(k)} \int_{\zeta^{(k)}}(y) \eta^{\left(j^{\prime \prime}\right)}(y)\left\{\Pi_{\bar{y}, \bar{\xi}, \hat{\xi}}^{y}\left[\widetilde{a}_{s}\left(\xi^{\left(j^{\prime \prime}\right)}, \tau ; \overline{\bar{y}}^{\prime}\right) D_{\bar{y}}^{=s} \overline{\bar{U}}_{j^{\prime \prime}}^{(0)}\left(\overline{\bar{y}}, \tau_{0} ; \hat{\bar{\xi}}, \tau\right)\right]\right.
$$




$$
\left.+\left[a_{s}(\xi, \tau)-a_{s}\left(\xi^{\left(j^{\prime \prime}\right)}, \tau\right)\right] D_{y}^{s} U_{j^{\prime \prime}}^{(0)}\left(y, \tau_{0} ; \xi, \tau\right)\right\} d y d \tau_{0} \mid
$$

As for $I$, it is sufficient to estimate

$$
\text { (4. 49) } \begin{aligned}
I^{\prime \prime}= & \iint_{K_{1}} \bar{\zeta}^{\left(k^{\prime \prime}\right)}(\bar{y}) \bar{\eta}^{\left(j^{\prime \prime}\right)}(\bar{y})\left\{\Pi _ { \overline { y } , \overline { \xi } , \overline { \xi } } ^ { \overline { y } } \left[\widetilde{a}_{s}\left(\xi^{\left(j^{\prime \prime}\right)}, \tau: \overline{\bar{y}}^{\prime}\right) D_{\bar{y}^{s}} \overline{\bar{U}}\left(j_{j^{\prime \prime}}^{(0)}(\overline{\bar{y}}, \tau ; \hat{\xi}, \tau)\right]\right.\right. \\
& \left.+\left[a_{s}(\xi, \tau)-a_{s}\left(\xi^{\left(j^{\prime \prime}\right)}, \tau\right)\right] \Pi_{y}^{\bar{y}} D_{y}^{s} U_{j^{\prime \prime}}^{(0)}\left(y, \tau_{0} ; \hat{\xi}, \tau\right)\right\} d \bar{y} d \tau_{0},
\end{aligned}
$$

where $K_{1}=\left\{\left(\bar{y}, \tau_{0}\right) \in K \times[\tau, t]|| y-\xi|+| \tau_{0}-\left.\tau\right|^{1 / 2} \leqq \frac{1}{2}(t-\tau)^{1 / 2}\right\}$. It is to be noted that the coordinates $\bar{y}$ and $\overline{\bar{y}}$ of $y \in \Omega^{\left(k^{\prime \prime}\right)} \cap \Omega^{\left(j^{\prime \prime}\right)}$ are connected with $\overline{\bar{y}}=h_{1}(\bar{y})$ and $\bar{y}=h_{2}(\overline{\bar{y}})$ having the same smoothness and $F$ is independent of $k^{\prime \prime}$ and $j^{\prime \prime}$. The integrand of (4.49) can be expressed as follows:

(4.50) $\quad \Pi_{\frac{\bar{y}}{\bar{y}}, \hat{\xi} \hat{\xi}}^{\overline{\hat{\xi}}} D_{\bar{y}}^{-s} \overline{\bar{U}}\left(\bar{j}^{\prime \prime}\left(\overline{\bar{y}}, \tau_{0} ; \hat{\xi}, \tau\right)\right.$

$$
=\Pi_{\hat{\xi}}^{\xi} \sum_{|\nu| \leqq|s|} \tilde{b}_{s \nu}(\bar{y}) D_{\bar{y}^{\nu}} \overline{\bar{U}}_{j^{\prime \prime}}^{(0)}\left(h_{1}(\bar{y}), \tau_{0} ; \bar{h}_{1}(\bar{\xi}), \tau\right)
$$

where $\tilde{b}_{s v}(\bar{y})$ depend on the function $h_{1}$ and the derivatives of $h_{2}$ and $\hat{\xi}=\widehat{h}_{1}(\hat{\xi})$, and

(4.51) $\quad \Pi_{y}^{\bar{y}} D_{y}{ }^{s} U_{j^{\prime \prime}}^{(0)}\left(y, \tau_{11} ; \xi, \tau\right)$

$$
=\Pi_{\bar{\xi}}{ }_{|\nu| \leqq|s|} \sum_{\mid s \nu} \tilde{c}_{s \nu}(\bar{y}) D_{\bar{y}^{\nu}} \overline{\bar{U}}_{j^{\prime \prime}}^{(0)}\left(h_{1}(\bar{y}), \tau_{0} ; \dot{h_{1}}(\bar{\xi}), \tau\right),
$$

where $\tilde{c}_{s \nu}(\bar{y})$ depend on $F^{\left(k^{\prime \prime}\right)}, h_{1}$ and the derivatives of $F^{\left(j^{\prime \prime}\right)}$ and $h_{2}$. $F^{\left(k^{\prime \prime}\right)}$ is a representation of $F$ by $k^{\prime \prime}$-local coordinate system. From (4. 49)(4.51) it follows that

$$
\begin{aligned}
I^{\prime \prime}= & \sum_{|\nu| \leqq|s|} \Pi_{\bar{\xi}^{\xi}} \iint_{K_{1}} \bar{\zeta}^{\left(k^{\prime \prime}\right)}(\bar{y}) \bar{\eta}^{\left(j^{\prime \prime}\right)}(\bar{y})\left\{\widetilde{a}_{s}\left(\xi^{\left(j^{\prime \prime}\right)}, \tau ; h_{1}{ }^{\prime}(\bar{y})\right) \tilde{b}_{s \nu}(\bar{y})\right. \\
& \left.+\left[\widetilde{a}_{s}(\xi, \tau)-\widetilde{a}_{s}\left(\xi^{\left(j^{\prime \prime}\right)}, \tau\right)\right] \tilde{c}_{s \nu}(\bar{y})\right\} \\
& \times D_{\bar{y}^{\nu}} \overline{\bar{U}}_{j^{\prime \prime}}^{(0)}\left(h_{1}(\bar{y}), \tau_{0} ; \widehat{h}_{1}(\hat{\xi}), \tau\right) d \bar{y} d \tau_{0} .
\end{aligned}
$$

If $|\nu|<2$, then

$$
\left|I^{\prime \prime}\right| \leqq C_{35} C_{36}^{(6)} \Lambda \rho_{d_{5}}(t, \tau ; \xi) .
$$

If $|\nu|=2, \nu_{3}<2$ then

$$
\left|I^{\prime \prime}\right| \leqq \mid \int_{K_{1}} \int_{\bar{y}^{\prime}}\left[\overline { \zeta } ^ { ( k ^ { \prime \prime } ) } ( \overline { y } ) \overline { \eta } ^ { ( j ^ { \prime \prime } ) } ( \overline { y } ) \left\{\widetilde{a}_{s}\left(\xi^{\left(j^{\prime \prime}\right)}, \tau ; h_{1}^{\prime}(\bar{y})\right) \tilde{b}_{s \nu}(\bar{y})\right.\right.
$$




$$
\begin{aligned}
& \left.\left.+\left[a_{s}(\xi, \tau)-a_{s}\left(\xi^{\left(j^{\prime \prime}\right)}, \tau\right)\right] \tilde{c}_{s \nu}(\bar{y})\right\}\right] D_{\bar{y}} \overline{\bar{U}}_{j^{\prime \prime}}^{(0)} d \bar{y} d \tau_{0} \mid \\
& +\mid \int_{\Gamma_{1}} \bar{\zeta}^{\left(k^{\prime \prime}\right)}(\bar{y}) \bar{\eta}^{\left(j^{\prime \prime}\right)}(\bar{y})\left\{\widetilde{a}_{s} \tilde{b}_{s \nu}+\left[a_{s}(\xi, \tau)-a_{s}\left(\xi^{\left(j^{\prime \prime}\right)}, \tau\right)\right] \tilde{c}_{s \nu}\right\} \\
& \times D_{\bar{y}} \overline{\bar{U}}_{j^{\prime \prime}}^{(0)} \cos \left(n, \bar{y}^{\prime}\right) d \Gamma_{1} \mid \\
& \leqq C_{35} C_{36}^{(7)} \Lambda \rho_{d_{5}}(t, \tau ; \xi) .
\end{aligned}
$$

If $|\nu|=\nu_{3}=2$, then we can reduce to the two previous cases. Thus we have

$$
u_{0}^{(1)} \in H_{d_{s}}^{\alpha, 2}\left(Q_{\tau, \tau+h}, \bar{Q}_{T}\right),\left\|u_{0}^{(1)}\right\| \leqq C_{35} C_{36} \Lambda
$$

By induction we have

$$
u_{0}^{(\nu)} \in H_{d_{5}}^{\alpha, 2}\left(Q_{\tau, \tau+h}, \bar{Q}_{T}\right),\left\|u_{0}{ }^{(\nu)}\right\| \leqq C_{35} C_{36}^{\nu} \Lambda^{\nu} \quad(\nu=0,1,2, \cdots)
$$

Choosing $\lambda, \chi$ and hence $h$, sufficiently small, we obtain that $u_{0}$ $=\Sigma u_{0}{ }^{(\nu)}$ converges uniformly in $Q_{\tau, \tau+h}$. (4.42), (4.43) and (4.56) imply that the assertion of the lemma holds.

Lemma 4. 8. $G(x, t ; \xi, \tau ; w) \in U_{C_{37}, d_{5}}^{2+\alpha, 0,0}\left(Q_{\tau, \tau+h}, \bar{Q}_{T}\right)$.

Proof. It is obvious from (4.41) and Lemmas 4.3, 4. 4, 4.7. Q.E.D.

Lemma 4. 9. $G \in U_{C_{37}, \dot{d}_{6}}^{2+\alpha, 0,0}\left(Q_{\tau, T}, \bar{Q}_{T}\right) \quad\left(d_{6}=d_{5} / 2\right)$.

Proof. The existence of the unique solution $G_{0}$ for (3.39) belonging to $C_{x, t}^{2+\alpha,(1+\alpha) / 2}\left(\bar{Q}_{r, T}\right)$ can easily be proved by the usual procedures. Thus we only have to estimate $G_{0}$ for $t>\tau+h$.

Let $\tau_{1}=\tau+\frac{h}{2}$ and let $\chi_{h}(t)$ be a smooth function such that

$$
\chi_{h}(t)=\left\{\begin{array}{lll}
0, & \text { if } & t \leqq h / 6, \\
1, & \text { if } & t \geqq h / 3 .
\end{array}\right.
$$

Then $\omega\left(x, t ; \xi, \tau ; \tau_{1}\right) \equiv \chi_{h}\left(t-\tau_{1}\right) G_{0}(x, t ; \xi, \tau ; w)$ satisfies the system of equations : 


$$
\left\{\begin{array}{l}
\left(D_{t}-\mathfrak{A}\right) \omega=\chi_{h}{ }^{\prime}\left(t-\tau_{1}\right) G_{0}(x, t ; \xi, \tau ; w) \equiv g_{0}\left(x, t ; \xi, \tau ; \tau_{1}\right), \\
\left.\omega\right|_{t=\tau_{1}}=0,\left.\quad \omega\right|_{\Gamma_{T}}=\left.\chi_{h}\left(t-\tau_{1}\right) Z\right|_{\Gamma_{T}} \equiv g_{1}\left(x, t ; \xi, \tau ; \tau_{1}\right) .
\end{array}\right.
$$

$\omega$ is obtained as a solution of (4.57) by the uniqueness of the solution. The property of $\chi_{h}$ and (4.41) imply that $g_{0}=0$ for $t<\tau_{1}+\frac{h}{6}$ and $t>\tau_{1}+\frac{h}{3}$. Since

$$
G_{0} \in H_{d_{s}}^{2+\alpha, 0}\left(Q_{\tau, \tau+h}, \bar{Q}_{T}\right),
$$

we have $g_{0} \in{\stackrel{\circ}{d_{5}}}_{d_{5}}^{\alpha}\left(\bar{Q}_{\tau_{1}, T}, \bar{Q}_{T}\right)$. For (4.57), utilizing Lemmas 4.3-4.5 and setting $u_{j}{ }^{(0)}=g_{j}(j=0,1)$, we can prove in the same way as in the proof of Lemma 4.7 that the solution $\omega$ of (4.57) defined in $\bar{Q}_{\tau_{1}, \tau_{1}+h_{0}}$ for some $h_{0}$ uniquely exists and belongs to ${\stackrel{\circ}{d_{s}}}^{2+\alpha}\left(\bar{Q}_{\tau_{1}, r_{1}+h_{0}}, \bar{Q}_{T}\right)$. Since $\omega=G_{0}$ for $t>\tau_{1}+h / 3, G_{0} \in \hat{H}_{d_{5}}^{2+\alpha}\left(\bar{Q}_{\tau_{1}, \tau_{1}+h_{0}}, \bar{Q}_{T}\right)$. It is to be noted that $h_{0}$ is independent of $\tau_{1}$.

In the next place, if we put $\omega\left(x, t ; \xi, \tau ; \tau_{2}\right)=\chi_{h_{0}}\left(t-\tau_{2}\right) G_{0}(x, t ; \xi, \tau$; $w)\left(\tau_{2}=\tau_{1}+h_{0} / 2\right)$, then $\omega$ is a solution of a system of equations analogous to (4.57). Hence $G_{0} \in \hat{H}_{d_{5}}^{2+\alpha}\left(\bar{Q}_{r_{1}, \tau_{2}+h_{0}}, \bar{Q}_{T}\right)$.

After a finite number of repetitions of this procedure, we obtain $G_{0} \in \hat{H}_{d_{s}}^{2+\alpha}\left(\bar{Q}_{\tau_{1}, T}, \bar{Q}_{T}\right)$. From this and (4.58) we deduce $G_{0} \in H_{d_{\mathrm{s}}}^{2+\alpha, 0}\left(Q_{\tau}, T\right.$, $\left.\bar{Q}_{T}\right)$.

Q.E.D.

The following lemma is an immediate consequence of Lemma 4.9.

\section{Lemma 4. 10.}

(4. 59)

$$
\begin{aligned}
& \left(\left|D_{t}^{r} D_{x}^{s} G\right| \leqq C_{38}^{(r,|s|)}(t-\tau)^{-(3+2 r+|s|) / 2}\right. \\
& \times \exp \left[-d_{7} \frac{|x-\xi|^{2}}{t-\tau}\right](2 r+|s| \leqq 2), \\
& \left|\Delta_{x}{ }^{x_{0}} D_{t}^{r} D_{x}^{s} G\right| \leqq C_{39}\left|x-x_{0}\right|^{\alpha}(t-\tau)^{-(5+\alpha) / 2} \\
& \times \exp \left[-d_{7} \frac{\left|x^{\prime \prime}-\xi\right|^{2}}{t-\tau}\right](2 r+|s|=2), \\
& \left|\Delta_{t}{ }^{t_{0}} D_{t}^{r} D_{x}^{s} G\right| \leqq C_{40}^{(r,|s|)}\left(t-t_{0}\right)^{(2-2 r-|s|+\alpha) / 2}\left(t_{0}-\tau\right)^{-(5+\alpha) / 2} \\
& \times \exp \left[-d_{7} \frac{|x-\xi|^{2}}{t-\tau}\right]\left(t>t_{0}>\tau, 0<2 r+|s| \leqq 2 ; d_{7}=d_{6} / 2\right) .
\end{aligned}
$$


Remark. As for $G_{0}$, the same estimates hold with $|x-\xi|+d(\xi, \Gamma)$ and $\left|x^{\prime \prime}-\xi\right|+d(\xi, \Gamma)$ in place of $|x-\xi|$ and $\left|x^{\prime \prime}-\xi\right|$, respectively.

\section{$\S 5$. Estimates for the Solution in $H^{2+\alpha}\left(\bar{Q}_{T}\right)$ of a Linear Problem (2. 17)}

The unique solution of a linear system $(2.17)$ in $H^{2+\alpha}\left(\bar{Q}_{T}\right)$ is given by

$$
\widetilde{w}(x, t)=\int_{0}^{t} d \tau \int_{\Omega} G(x, t ; \xi, \tau ; w) \phi(\xi, \tau, w) d \xi,
$$

for (5.1) certainly satisfies (2.17) and belongs to $H^{2+\alpha}\left(\bar{Q}_{T}\right)$ as we shall see below.

Let $\varepsilon$ and $h$ be sufficiently small positive numbers. We introduce the notations

$$
U_{\varepsilon}\left(x^{0}\right)=\bar{\Omega} \cap\left\{\left|x-x^{0}\right| \leqq \varepsilon\right\}, Q_{h \varepsilon}\left(x^{0}, t^{0}\right)=U_{\varepsilon}\left(x^{0}\right) \times\left[t^{0}+h, t^{0}+2 h\right] .
$$

Then in order to prove $\widetilde{w} \in H^{2+\alpha}\left(\bar{Q}_{T}\right)$, it is sufficient to show that $\widetilde{w} \in H^{2+\alpha}\left(Q_{h \varepsilon}\left(x^{0}, t^{0}\right)\right)$ for $\forall\left(x^{0}, t^{0}\right) \in \bar{\Omega} \times[-h, T-2 h]$ and $\|\widetilde{w}\|_{Q_{h \varepsilon}\left(x^{0}, t^{0}\right)}^{(2+\alpha)}$ $\leqq C_{41}\|\phi\|_{T}{ }^{(\alpha)}$, where $C_{41}$ is a constant independent of $\left(x^{0}, t^{0}\right)$.

\section{Lemma 5. 1 .}

$$
\begin{aligned}
& \left|D_{x}^{s} \widetilde{\widetilde{w}}\right| \leqq C_{42}^{(|s|)} t^{(2-|s|+\alpha) / 2}\|\phi\|_{T}^{(\alpha)} \quad \text { if } \quad Q_{h \varepsilon}\left(x^{0}, t^{0}\right) \cap \Gamma_{T}=\varnothing \quad(|s| \leqq 2), \\
& \left|D_{x}^{s} \widetilde{\widetilde{w}}\right| \leqq \bar{C}_{42}^{(|s|)} t^{(2-|s|) / 2}\left(t+\left|x-x^{*}\right|^{2}\right)^{\alpha / 2}\|\phi\|_{T}^{(\alpha)}
\end{aligned}
$$

$$
\text { if } Q_{h \varepsilon}\left(x^{0}, t^{0}\right) \cap \Gamma_{T} \neq \varnothing(|s| \leqq 2),
$$

where $x^{*}$ is a nearest boundary point of $x$,

$$
\begin{aligned}
& \left|\Delta_{x}{ }^{x_{0}} D_{x}^{s} \widetilde{\boldsymbol{w}}\right| \leqq C_{43}\left|x-x_{0}\right|^{\alpha}\|\phi\|_{T}^{(\alpha)} \quad(|s|=2), \\
& \left|\Delta_{t}^{t_{0}} D_{x}^{s} \widetilde{\widetilde{w}}\right| \leqq C_{44}^{(|s|)}\left(t-t_{0}\right)^{(2-|s|+\alpha) / 2}\|\phi\|_{T}{ }^{(\alpha)}(0<|s| \leqq 2) .
\end{aligned}
$$

Proof. Let $\chi_{0}(\tau)$ and $\chi_{1}(\xi)$ be smooth functions on $R^{1}$ and $R^{3}$, respectively, such that $0 \leqq \chi_{0}, \chi_{1} \leqq 1$ and

$$
\chi_{0}(\tau)=\left\{\begin{array}{ccc}
1 & \text { if } & \tau \geqq \frac{2}{3} h \\
0 & \text { if } & \tau \leqq \frac{1}{2} h
\end{array}, \quad \chi_{1}(\xi)=\left\{\begin{array}{ccc}
1 & \text { if } & |\xi| \leqq \frac{3}{2} \varepsilon \\
0 & \text { if } & |\xi| \geqq 2 \varepsilon
\end{array} .\right.\right.
$$


$\widetilde{w}$ is transformed into the form:

(5. 7) $\widetilde{w}(x, t)=\int_{0}^{t} d \tau \int_{\Omega} G \phi \chi(\xi, \tau) d \xi+\int_{0}^{t} d \tau \int_{\Omega} G \phi(1-\chi) d \xi=\widetilde{w}_{1}+\widetilde{w}_{2}$, where $\chi(\xi, \tau)=\chi_{0}\left(\tau-t^{0}\right) \chi_{1}\left(\xi-x^{0}\right)$. It is easily seen that

$$
\left\{\begin{array}{l}
\left|D_{x}^{s} \widetilde{\widetilde{w}}_{2}\right| \leqq C_{42,2}^{(j s)} t^{(2-|s|+\alpha) / 2}\|\phi\|_{T}{ }^{(\alpha)} \quad(|s| \leqq 2), \\
\left|\Delta_{x}^{x_{0}} D_{x}^{s} \widetilde{\mathfrak{w}}_{2}\right| \leqq C_{43,2}\left|x-x_{0}\right|^{\alpha}\|\phi\|_{T}{ }^{(\alpha)} \quad(|s|=2) \\
\left|\Delta_{t}^{t_{0}} D_{x}^{s} \widetilde{\widetilde{w}}_{2}\right| \leqq C_{44,2}^{(|s|)}\left(t-t_{0}\right)^{(2-|s|+\alpha) / 2}\|\phi\|_{T}{ }^{(\alpha)} \quad(0<|s| \leqq 2)
\end{array}\right.
$$

As for $\widetilde{w}_{1}$, we shall estimate only when $x^{0} \in \Gamma, t^{0}=-h$. Other cases can be estimated similarly to this case more easily because of less singularities. For simplicity $U_{2 \varepsilon}\left(x^{0}\right)$ and $Q_{h \varepsilon}\left(x^{0},-h\right)$ are denoted by $U^{+}$and $Q_{0}$, respectively. By (3.38) $\widetilde{w}_{1}$ can be expressed in the following form:

$$
\begin{gathered}
\widetilde{w}_{1}=\int_{0}^{t} d \tau \int_{U^{+}} Z_{\phi \chi_{1}}\left(\xi-x^{0}\right) d \xi-\int_{0}^{t} d \tau \int_{U^{+}} G_{0} \phi \chi_{1}\left(\xi-x^{0}\right) d \xi \\
=\widetilde{\widetilde{w}}_{1,1}-\widetilde{\widetilde{\omega}}_{1,2} .
\end{gathered}
$$

(5. 10) $\quad \widetilde{\widetilde{w}}_{1,1}=\int_{0}^{t} d \tau \int_{R s} Z^{*} \phi \chi_{1} d \xi-\int_{0}^{t} d \tau \int_{U^{-}} Z^{*} \phi \chi_{1} d \xi=\widetilde{\mho}_{1,1,1}-\widetilde{\mho}_{1,1,2}$,

where $U^{-}=\left(R^{3}-\Omega\right) \cap\left\{\left|\xi-x^{0}\right| \leqq 2 \varepsilon\right\}$ (as for ${ }^{*} \phi$, see Lemma 3.1). It is well known that (cf. $[13,25]$ )

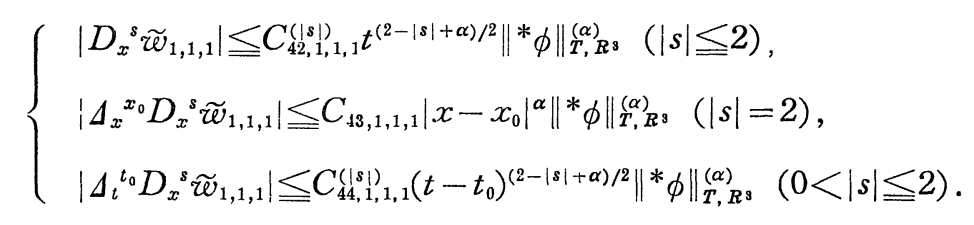

Since $\phi\left(x^{*}, 0\right)=0$, we have for $|s| \leqq 2$

(5. 12) $\left|D_{x}^{s} \widetilde{\mho}_{1,1,2}\right| \leqq C_{42,1,1,2}^{(|s|)} t^{(2-|s|) / 2}\left(t^{\alpha / 2}+\left|x-x^{*}\right|^{\alpha}\right)\left\|^{*} \phi\right\|_{T^{T}, R^{s}}^{(\alpha)}\left(1+t^{-(2-|s|) / 2}\left|I_{s}\right|\right)$, where

$$
I_{s}=\int_{0}^{t} d \tau \int_{U^{-}} D_{x}^{s} Z_{\chi_{1}} d \xi
$$

After some lengthy calculations, we obtain

$$
\left|I_{s}\right| \leqq C_{4 !}^{(|s|)} t^{(2-|s|) / 2}
$$

therefore 


$$
\left|D_{x}^{s} \widetilde{w}_{1,1,2}\right| \leqq \bar{C}_{42,1,1,2}^{(|s|)}\left(t+\left|x-x^{*}\right|^{2}\right)^{(2-|s|+\alpha) / 2}\left\|^{*} \phi\right\|_{T_{R}, R^{3}}^{(\alpha)}
$$

For $t-t_{0} \geqq t_{0}+\left|x-x^{*}\right|^{2}$ we immediately derive from (5.14) that

$$
\left|\Delta_{t}^{t_{0}} D_{x}^{s} \widetilde{w}_{1,1,2}\right| \leqq C_{44,1,1,2}^{(|s|)}\left(t-t_{0}\right)^{(2-|s|+\alpha) / 2}\left\|^{*} \phi\right\|_{T^{\prime}, R^{s}}^{(\alpha)} .
$$

For $t-t_{0}<t_{0}+\left|x-x^{*}\right|^{2}$ we put $t^{\prime \prime}=\max \left\{2 t_{0}-t, 0\right\}$ and then obtain

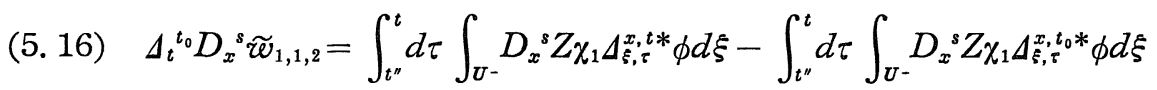

$$
\begin{aligned}
& +\int_{0}^{t^{\prime \prime}} d \tau \int_{U^{-}} \Delta_{t}^{t_{0}} D_{x}^{s} Z_{\chi_{1}} \Delta_{\xi, \tau}^{x, t_{0} *} \phi d \xi+\Delta_{t}^{t_{0} *} \phi \int_{t^{\prime \prime}}^{t} d \tau \int_{U^{-}} D_{x}^{s} Z \chi_{1} d \xi \\
& +\Delta_{x, i_{0}}^{x^{*}, 0 *} \phi \Delta_{t}^{t_{0}} \int_{0}^{t} d \tau \int_{U^{-}} D_{x}^{s} Z_{\chi_{1}} d \xi \equiv \sum_{i=1}^{5} J_{i}^{(1)} .
\end{aligned}
$$

$J_{i}^{(1)}(i=1,2,3)$ can directly be estimated by Lemma 3.6. As for $J_{4}^{(1)}$ we can derive the desired estimate from the following estimate obtained similarly to $I_{s}$

$$
\left|\int_{t^{\prime \prime}}^{t} d \tau \int_{U^{-}} D_{x}^{s} Z \chi_{1} d \xi\right| \leqq C_{46}^{(|s|)}\left(t-t^{\prime \prime}\right)^{(2-|s|) / 2}
$$

In order to estimate $J_{5}^{(1)}$ we transform this into the form

$$
\begin{aligned}
& \Delta_{t}^{t_{0}} \int_{0}^{t} d \tau \int_{U^{-}} D_{x}^{s} Z_{\chi_{1}} d \xi=\Delta_{t}^{t_{0}} \int_{0}^{t} d \tau \int_{U^{-}} D_{x}^{s} Z_{0} \chi_{1} d \xi \\
& +\Delta_{t}^{t_{0}} \int_{0}^{t} d \tau \int_{U^{-}} D_{x}^{s} Z^{\prime} \chi_{1} d \xi \equiv J_{5,1}^{(1)}+J_{5,2}^{(1)} . \\
& J_{5,1}^{(1)}=\int_{t^{\prime \prime}}^{t} d \tau \int_{U^{-}}\left[D_{x}^{s} Z_{0}(x-\xi, t-\tau ; \xi, \tau ; w)\right. \\
& \left.-\left.D_{x}^{s} Z_{0}(x-\xi, t-\tau ; z, t ; w)\right|_{z=x}\right] \chi_{1} d \xi \\
& -\int_{t^{\prime \prime}}^{t_{0}} d \tau \int_{U^{-}}\left[D_{x}^{s} Z_{0}\left(x-\xi, t_{0}-\tau ; \xi, \tau ; w\right)\right. \\
& \left.-D_{x}^{s} Z_{0}\left(x-\xi, t-\tau ; z, t_{0} ; w\right)_{\mid z=x}\right] \chi_{1} d \xi \\
& +\int_{0}^{t^{\prime \prime}} d \tau \int_{U^{-}} \Delta_{t}^{t_{0}}\left[D_{x}^{s} Z_{0}(x-\xi, t-\tau ; \xi, \tau ; w)\right. \\
& \left.-D_{x}^{s} Z_{0}\left(x-\xi, t-\tau ; z, t_{0} ; w\right)_{\mid z=x}\right] \chi_{1} d \xi \\
& +\int_{t^{\prime \prime}}^{t} d \tau \int_{U^{-}}\left[D_{x}^{s} Z_{0}(x-\xi, t-\tau ; z, t ; w)\right. \\
& \left.-D_{x}^{s} Z_{0}\left(x-\xi, t-\tau ; z, t_{0} ; w\right)\right]_{\mid z=x} \chi_{1} d \xi
\end{aligned}
$$




$$
+\Delta_{t}^{t_{0}} \int_{0}^{t} d \tau \int_{U^{-}} D_{x}^{s} Z_{0}\left(x-\xi, t-\tau ; z, t_{0} ; w\right)_{\mid z=x} \chi_{1} d \xi \equiv \sum_{i=1}^{5} J_{5,1, i}^{(1)} .
$$

We easily obtain by Lemma 3.4

$$
\left|J_{5,1, i}^{(1)}\right| \leqq C_{47,1, i}^{(|s|)}\left(t-t_{0}\right)^{(2-|s|+\alpha) / 2} \quad(i=1,2,3,4) .
$$

$$
J_{5,1,5}^{(1)}=-\Delta_{t}^{t_{0}} \int_{0}^{t} d \tau \int_{U^{-}} D_{\xi}^{s} Z_{0}\left(x-\xi, t-\tau ; x, t_{0} ; w\right) \chi_{1} d \xi
$$

In an analogous way as $I_{s}$ we have

$$
\left|J_{5,1,5}^{(1)}\right| \leqq C_{47,1,5}^{(i s \mid)}\left(t-t_{0}\right)^{(2-|s|+\alpha) / 2} .
$$

As for $J_{5,2}^{(1)}$, we rewrite $J_{5,2}^{(1)}$ according to the definition of $Z^{\prime}$.

$$
J_{5,2}^{(1)}=\Delta_{t}^{t_{0}} D_{x}^{s} \int_{0}^{t} d \tau_{0} \int_{R^{s}} Z_{0}\left(x-y, t-\tau_{0} ; y, \tau_{0} ; w\right) \Psi\left(y, \tau_{0}\right) d y,
$$

where

$$
\Psi\left(y, \tau_{0}\right)=\int_{0}^{\tau_{0}} d \tau \int_{U^{-}} \Phi\left(y, \tau_{0} ; \xi, \tau ; w\right) \chi_{1}\left(\xi-x^{0}\right) d \xi
$$

The fact that $\Psi \in H^{\alpha}\left(\bar{R}_{T}{ }^{3}\right)$, which is proved by the same arguments as above, implies that

$$
\left|J_{5,2}^{(1)}\right| \leqq C_{47,2}^{(|s|)}\left(t-t_{0}\right)^{(2-|s|+\alpha) / 2}
$$

Hence we have

$$
\left|\Delta_{t}^{t_{0}} D_{x}^{s} \widetilde{\mathfrak{w}}_{1,1,2}\right| \leqq \bar{C}_{44,1,1,2}^{(|s|)}\left(t-t_{0}\right)^{(2-|s|+\alpha) / 2}\left\|^{*} \phi\right\|_{T^{\prime}, R^{s}}^{(\alpha)}
$$

Next, for clearness' sake, we assume that $x^{\prime \prime}=x_{0}$.

$$
\begin{aligned}
\Delta_{x} x_{0} D_{x}^{s} \widetilde{w}_{1,1,2} & =\int_{0}^{t} d \tau \int_{\sigma} D_{x}^{s} Z(x-\xi, t-\tau ; \xi, \tau ; w) \chi_{1} \Delta_{\xi, \tau}^{x_{0, t} t *} \phi d \xi \\
& -\int_{0}^{t} d \tau \int_{\sigma} D_{x_{0}}^{s} Z\left(x_{0}-\xi, t-\tau ; \xi, \tau ; w\right) \chi_{1} \Delta_{\xi, \tau}^{x_{0}, t *} \phi d \xi \\
& +\int_{0}^{t} d \tau \int_{U^{-}-\sigma} \Delta_{x}^{x_{0}} D_{x}^{s} Z \chi_{1} \Delta_{\xi, \tau}^{x_{0}, t *} \phi d \xi \\
& +\int_{0}^{t} d \tau \int_{U^{-}} \Delta_{x}^{x_{0}} D_{x}^{s} Z \chi_{1} d \xi \cdot \Delta_{x_{0}, t}^{x^{\alpha^{*}}, t *} \phi,
\end{aligned}
$$

where $\sigma=U^{-} \cap\left\{|\xi-x| \leqq 2\left|x-x_{0}\right|\right\}$ and $x_{0}{ }^{*}$ is a nearest boundary point of $x_{0}$. Applying Lemma 3.6 to the first three integrals and using the same procedures as $(5.18)-(5.23)$ we have 


$$
\left|\Delta_{x}^{x_{0}} D_{x}^{s} \widetilde{w}_{1,1,2}\right| \leqq C_{43,1,1,2}\left|x-x_{0}\right|^{\alpha}\left\|^{*} \phi\right\|_{T, R s}^{(\alpha)} \quad(|s|=2) .
$$

Last of all, let us estimate $\widetilde{w}_{1,2}$. Let $\varepsilon$ be sufficiently small such that $U^{+} \cap \Omega^{\left(k^{\prime}\right)}=\varnothing$ for any $k^{\prime}$. For arbitrary $(x, t) \in Q_{0}$,

$$
\begin{aligned}
& G_{0}(x, t ; \xi, \tau ; \tau)=\sum_{k^{\prime \prime}} \eta^{\left(k^{\prime \prime}\right)}(x) \\
& \quad \times \Pi_{\bar{x}}{ }^{x}\left[\int_{\tau}^{t} d \tau_{0} \int_{K} H_{0}{ }^{\left(k^{\prime \prime}\right)}\left(\bar{x}, t ; \bar{y}, \tau_{0}\right) \bar{\zeta}^{\left(k^{\prime \prime}\right)}(\bar{y}) \bar{u}_{0}\left(\bar{y}, \tau_{0} ; \xi, \tau\right) d \bar{y}\right. \\
& \left.\quad+\int_{\tau}^{t} d \tau_{0} \int_{K^{\prime}} H_{1}{ }^{\left(k^{\prime \prime}\right)}\left(\bar{x}-\bar{y}^{\prime}, t-\tau_{0}\right) \bar{\zeta}^{\left(k^{\prime \prime}\right)}\left(\bar{y}^{\prime}\right) \bar{u}_{1}\left(\bar{y}^{\prime}, \tau_{0} ; \xi, \tau\right) d \bar{y}^{\prime}\right]
\end{aligned}
$$

(cf. §3.4),

hence $\widetilde{w}_{1,2}$ can be expressed in the form

$$
\begin{aligned}
& \widetilde{w}_{1,2}(x, t)=\sum_{k^{\prime \prime}} \eta^{\left(k^{\prime \prime}\right)}(x) \\
& \quad \times \Pi_{\bar{x}}{ }^{x}\left[\int_{0}^{t} d \tau_{0} \int_{K} H_{0}{ }^{\left(k^{\prime \prime}\right)}\left(\bar{x}, t ; \bar{y}, \tau_{0}\right) \bar{\zeta}{ }^{\left(k^{\prime \prime}\right)}(\bar{y})\left(\bar{U}_{0} \phi\right)\left(\bar{y}, \tau_{0}\right) d \bar{y}\right. \\
& \left.\quad+\int_{0}^{t} d \tau_{0} \int_{K^{\prime}} H_{1}{ }^{\left(k^{\prime \prime}\right)}\left(\bar{x}-\bar{y}^{\prime}, t-\tau_{0}\right) \bar{\zeta}^{\left(k^{\prime \prime}\right)}\left(\bar{y}^{\prime}\right)\left(\bar{U}_{1} \phi\right)\left(\bar{y}^{\prime}, \tau_{0}\right) d \bar{y}^{\prime}\right],
\end{aligned}
$$

where

$$
\left\{\begin{array}{l}
\bar{U}_{j} \phi=\Pi_{y}^{\bar{y}} U_{j} \phi \quad(j=0,1) \\
U_{j} \phi=\int_{0}^{\tau_{0}} d \tau \int_{U^{+}} u_{j}(y, \tau ; \xi, \tau) \phi(\xi, \tau) \chi_{1}\left(\xi-x^{0}\right) d \xi
\end{array}\right.
$$

Assuming that

Lemma 5. 2. If $\phi \in C_{0}{ }^{\alpha}\left(Q_{T}\right)$, then $U_{0} \phi \in \stackrel{\circ}{C}^{\alpha}\left(\bar{Q}_{0, h}\right), U_{1} \phi \in \stackrel{\circ}{C}^{2+\alpha}\left(\Gamma_{0, h}\right)$ and

$$
\left\|U_{0} \phi\right\|_{\bar{Q}_{0, h}}^{(\alpha)} \leqq C_{48}\|\phi\|_{T}{ }^{(\alpha)},\|\| U_{1} \phi\|\|_{\Gamma_{0, h}}^{(2+\alpha)} \leqq C_{49}\|\phi\|_{T}{ }^{(\alpha)}
$$

holds and connecting this with Lemma 4.6, we obtain $\widetilde{\omega}_{1,2} \in \stackrel{C}{C}^{2+\alpha}\left(Q_{0}\right)$. Thus Lemma 5.1 is proved.

Q.E.D.

Now we proceed the proof of Lemma 5.2. In the same way as in the proof of Lemma 4.7 (cf. $\S 3.4$ )

$$
u_{0}\left(y, \tau_{0} ; \xi, \tau\right)=\sum_{\nu=0}^{\infty} u_{0}^{(\nu)}\left(y, \tau_{0} ; \xi, \tau\right), u_{1}\left(y, \tau_{0} ; \xi, \tau\right)=u_{1}^{(0)}\left(y, \tau_{0} ; \xi, \tau\right) .
$$


Hence we have

$$
U_{0} \phi=\sum_{\nu=0}^{\infty} U_{0}^{(\nu)} \phi, \quad U_{1} \phi=U_{1}^{(0)} \phi,
$$

where

$$
U_{j}^{(\nu)} \phi=\int_{0}^{\tau_{0}} d \tau \int_{U^{+}} u_{j}^{(\nu)}\left(y, \tau_{0} ; \xi, \tau\right) \phi(\xi, \tau) \chi_{1}\left(\xi-x^{0}\right) d \xi .
$$

From the estimates of $\widetilde{\widetilde{w}}_{1,1}$ in Lemma 5.1 follows that

$$
U_{1}{ }^{(0)} \phi \in \stackrel{C}{ }^{2+\alpha}\left(\Gamma_{0, h}\right), \quad\left\|U_{1}{ }^{(0)} \phi\right\|_{\Gamma_{0}, h}^{(2+a)} \leqq C_{49}\|\phi\|_{T}{ }^{(\alpha)} .
$$

Utilizing the expression of $u_{0}{ }^{(1)}$, Lemma 4.6 and (5.31), we obtain

$$
U_{0}^{(1)} \phi \in \stackrel{\complement}{C}^{\alpha}\left(\bar{Q}_{0, h}\right),\left\|U_{0}^{(1)} \phi\right\|\left\|_{\bar{Q}_{0, h}}^{(\alpha)} \leqq C_{33} C_{36} C_{49} \Lambda\right\| \phi \|_{T}{ }^{(\alpha)} .
$$

By induction we have

$$
U_{0}^{(\nu)} \phi \in \stackrel{\circ}{C}^{\alpha}\left(\bar{Q}_{0, h}\right),\left\|U_{0}{ }^{(\nu)}\right\|_{\bar{Q}_{0, h}}^{(\alpha)} \leqq C_{49} C_{33}^{\nu} C_{36}^{\nu} \Lambda^{\nu}\|\phi\|_{T}{ }^{(\alpha)} .
$$

Hence the lemma holds with $C_{48}=C_{49} /\left(1-C_{33} C_{36} \Lambda\right)$ for sufficiently small $h$. Q.E.D.

Corollary of Lemma 5. 1. For $(x, t) \in Q_{h \varepsilon}\left(x^{0}, t^{0}\right)$

$$
\left|D_{t} \widetilde{\tau}\right| \leqq C_{50}\left\{\begin{array}{l}
t^{\alpha / 2}\|\phi\|_{T}{ }^{(\alpha)}, \text { if } Q_{h \varepsilon}\left(x^{0}, t^{0}\right) \cap \Gamma_{T}=\varnothing, \\
\left(t+\left|x-x^{*}\right|^{2}\right)^{\alpha / 2}\|\phi\|_{T}{ }^{(\alpha)}, \text { otherwise. }
\end{array}\right.
$$

Lemma 5.3. $C_{42}^{(|s|)}, \bar{C}_{42}^{(|s|)}, C_{43}, C_{44}^{(|s|)}$ and $C_{50}$ are positive functions in $\langle w\rangle_{T}{ }^{(2, \alpha)},\left\|v_{0}\right\|^{(2+\alpha)},\left\|\theta_{0}\right\|^{(2+\alpha)},\left\|\theta_{1}\right\|_{T}{ }^{(2+\alpha)},\left\|\rho_{0}\right\|^{(1+\alpha)},\left(\bar{\rho}_{0}\right)^{-1}, \overline{\bar{\rho}}_{0},\left(\bar{\theta}_{0}\right)^{-1},(\bar{\sigma})^{-1}$, $\overline{\bar{\sigma}}$ and $T$ and monotonically increasing in each argument.

Proof. We obtain this by tracing the lengthy calculations made in $\S \S 2-5$.

Q.E.D.

The condition (2.18) implies that

$$
\mathfrak{P}_{i j}^{k m} \in H^{\alpha}\left(\bar{Q}_{T}\right),\left\|\mathfrak{P}_{i j}^{k m}\right\|_{T}{ }^{(\alpha)} \leqq b_{1}\left(T,\langle w\rangle_{T}{ }^{(2, \alpha)}\right) .
$$

From the definition of $g_{i j}$ it follows that

$$
\left\{\begin{array}{l}
\left|D_{x} g_{i j}\right|,\left|D_{t} g_{i j}\right|,\left|D_{x} g_{i j}\right|_{i, T}^{(\alpha / 2)} \leqq B_{3}\left(T,\langle w\rangle_{T}^{(2, \alpha)},\left\|v_{0}\right\|^{(2+\alpha)}\right) \\
\left|D_{x} g_{i j}\right|_{x, T}^{(\alpha)} \leqq B_{4}\left(T,\langle w\rangle_{T}{ }^{(2, \alpha)},\left\|v_{0}\right\|^{(2+\alpha)}\right) T\left|D_{x}^{2} w\right|_{T}{ }^{(\alpha)}
\end{array}\right.
$$


where $B_{3}$ has the same property as $B_{1}$.

Hence we obtain

$$
\left\{\begin{aligned}
&\left|\frac{D_{x} \hat{\rho}}{\hat{\rho}}\right| \leqq\left(\bar{\rho}_{0}\right)^{-1}\left|\rho_{0}\right|^{(0)}+3\left(1+3 B_{1}+3 B_{3}\right) T\left(\langle w\rangle_{T}{ }^{(2, \alpha)}+\left\|v_{0}\right\|^{(2+\alpha)}\right) \\
&\left|\frac{D_{x} \hat{\rho}}{\hat{\rho}}\right|_{x, T}^{(\alpha)} \leqq\left(\bar{\rho}_{0}\right)^{-2}\left|\rho_{0}^{\prime}\right|^{(0)}\left\{3 \overline{\bar{\rho}}_{0}+\left|\rho_{0}^{\prime}\right|^{(0)}\right\}+9 B_{4} T\left|D_{x}^{2} w\right|_{T}^{(\alpha)} \\
& \quad \times\left(\langle w\rangle_{T}^{(2, \alpha)}+\left\|v_{0}\right\|^{(2+\alpha)}\right)+3\left(1+3 B_{2}\right) T\left(\left|D_{x}{ }^{2} w\right|_{T}^{(\alpha)}\right. \\
&\left.\quad+\left\|v_{0}\right\|^{(2+\alpha)}\right) \\
&\left|\frac{D_{x} \hat{\rho}}{\hat{\rho}}\right|_{t, T}^{(\alpha / 2)} \leqq 3\left(1+B_{1}+B_{3}\right)\left(\langle w\rangle_{T}^{(2, \alpha)}+\left\|v_{0}\right\|^{(2+\alpha)}\right)
\end{aligned}\right.
$$

$$
\begin{aligned}
& \|\sigma\|_{T}{ }^{(1+\alpha)} \leqq\|\sigma\|_{T}{ }^{(2)}\|\hat{\rho}\|_{T}{ }^{(\alpha)}+\|\sigma\|_{T}{ }^{(2)}\|\hat{\theta}\|_{T}{ }^{(\alpha)} \\
& \left\{\begin{array}{c}
\left|f\left(x+\int_{0}^{t} \widehat{v} d \tau, t\right)-f\left(x_{0}+\int_{0}^{t} \widehat{v} d \tau, t\right)\right| \leqq\left|f_{x}\right|_{T}{ }^{(0)}\left|x-x_{0}\right| \\
\quad \times\left\{1+\left(\langle w\rangle_{T}^{(2, \alpha)}+\left\|v_{0}\right\|^{(2+\alpha)}\right) T\right\} \\
\left|f\left(x+\int_{0}^{t} \widehat{v} d \tau, t\right)-f\left(x+\int_{0}^{t_{0}} \widehat{v} d \tau, t_{0}\right)\right| \\
\leqq\left\{\left|f_{x}\right|_{T}^{(0)}\left(\langle w\rangle_{T}^{(2, \alpha)}+\left\|v_{0}\right\|^{(2+\alpha)}\right)+\left|f_{t}\right|_{T}^{(0)}\right\}\left(t-t_{0}\right)
\end{array}\right.
\end{aligned}
$$

From these follows that

$$
\begin{aligned}
& \mathfrak{B} \in H^{\alpha}\left(\bar{Q}_{T}\right), \\
& \|\mathfrak{B}\|_{T}{ }^{(\alpha)} \leqq b_{2}\left(T,\langle r\rangle_{T}{ }^{(2, \alpha)}\right)+T \cdot b_{3}\left(T,\langle w\rangle_{T}{ }^{(2, \alpha)}\right)\left|D_{x}{ }^{2} w\right|_{T}{ }^{(\alpha)} .
\end{aligned}
$$

From Lemma 5.1 with $\phi=\mathfrak{B}$ we derive

$$
\left\{\begin{array}{l}
\langle\widetilde{w}\rangle_{T}{ }^{(2, \alpha)} \leqq C_{51}\left(T,\langle w\rangle_{T}{ }^{(2, \alpha)}\right)\left(b_{2}+T \cdot b_{3}\left|D_{x}{ }^{2} w\right|_{T}{ }^{(\alpha)}\right), \\
\left|D_{x}{ }^{2} \widetilde{w}\right|_{T}{ }^{(\alpha)} \leqq C_{52}\left(T,\langle w\rangle_{T}{ }^{(2, \alpha)}\right)\left(b_{2}+T \cdot b_{3}\left|D_{x}{ }^{2} w\right|_{T}{ }^{(\alpha)}\right),
\end{array}\right.
$$

where $C_{51} \downarrow 0$ as $T \downarrow 0$. Therefore there exists $T_{1} \in(0, T]$ such that

$$
\left\{\begin{array}{l}
C_{51}\left(T_{1}, M_{1}\right) b_{2}\left(T_{1}, M_{1}\right)<M_{1} \\
C_{52}\left(T_{1}, M_{1}\right) b_{3}\left(T_{1}, M_{1}\right) T_{1}<1
\end{array}\right.
$$

Moreover there exists $T_{2} \in\left(0, T_{1}\right]$ such that

$$
C_{52}\left(T_{2}, M_{1}\right) b_{2}\left(T_{2}, M_{1}\right) \leqq \frac{1-C_{52}\left(T_{2}, M_{1}\right) b_{3}\left(T_{2}, M_{1}\right) T_{2}}{C_{51}\left(T_{2}, M_{1}\right) b_{3}\left(T_{2}, M_{1}\right) T_{2}}
$$




$$
\times\left\{M_{1}-C_{51}\left(T_{2}, M_{1}\right) b_{2}\left(T_{2}, M_{1}\right)\right\} .
$$

Hence there exists $M_{2}>0$ such that

(5. 44) $\frac{C_{52}\left(T_{2}, M_{1}\right) b_{2}\left(T_{2}, M_{1}\right)}{1-C_{52}\left(T_{2}, M_{1}\right) b_{3}\left(T_{2}, M_{1}\right) T_{2}} \leqq M_{2} \leqq \frac{M_{1}-C_{51}\left(T_{2}, M_{1}\right) b_{2}\left(T_{2}, M_{1}\right)}{C_{51}\left(T_{2}, M_{1}\right) b_{3}\left(T_{2}, M_{1}\right) T_{2}}$.

From (5.41) and (5.44) follows that

$$
\widetilde{w} \in \subseteq_{T_{2}}^{0} \equiv\left\{w \in \subseteq_{T_{2}}|| D_{x}^{2} w||_{T_{2}}^{(\alpha)} \leqq M_{2}\right\} .
$$

For simplicity we again choose $T=T_{2}$ from the beginning.

\section{$\S$ 6. The Existence of a Bounded Solution$$
\text { of }(2.15)-(2.16)
$$

We construct the sequence $\left\{w_{n}(x, t)\right\}$ of successive approximate solutions as follows:

$$
\left\{\begin{array}{l}
w_{0}(x, t)=0 \in \mathfrak{S}_{T}{ }^{0} \\
w_{n}(x, t) \text { is a solution of }(2.17) \text { with } \phi=\mathfrak{B}\left(x, t, w_{n-1}\right) \\
\text { assuming that } w=w_{n-1} \in \mathfrak{S}_{T}{ }^{0} .
\end{array}\right.
$$

Then by the results in $\S \S 2-5$ we have

(6. 1) $w_{n}(x, t)=\int_{0}^{t} d \tau \int_{\Omega} G\left(x, t ; \xi, \tau ; w_{n-1}\right) \mathfrak{B}\left(\xi, \tau, w_{n-1}\right) d \xi$,

which belongs to $\mathfrak{S}_{T}{ }^{0}$.

By induction we obtain

Lemma 6. 1. $w_{n} \in \Im_{T}^{0}$ for $n=0,1,2, \cdots$.

Next, let us consider the difference $w_{n}-w_{n-1}$, which satisfies the equality

$$
\left\{\begin{aligned}
D_{t}\left(w_{n}-w_{n-1}\right)=\mathfrak{P}\left(x, t, w_{n-1} ; D_{x}\right)\left(w_{n}-w_{n-1}\right) \\
\quad+\left\{\mathfrak{A}\left(x, t, w_{n-1} ; D_{x}\right)-\mathfrak{U}\left(x, t, w_{n-2} ; D_{x}\right)\right\} w_{n-1} \\
\quad+\mathfrak{B}\left(x, t, w_{n-1}\right)-\mathfrak{B}\left(x, t, w_{n-2}\right), \\
\left.\left(w_{n}-w_{n-1}\right)\right|_{t=0}=0,\left.\quad\left(w_{n}-w_{n-1}\right)\right|_{\Gamma_{T}}=0 .
\end{aligned}\right.
$$


From the definition of $g_{i j}$ it follows that

$$
\left\{\begin{array}{l}
\left\|g_{i j}\left(w_{n-1}\right)-g_{i j}\left(w_{n-2}\right)\right\|_{T}^{(1)} \\
\quad \leqq B_{5}\left(T,\left\langle w_{n-1}\right\rangle_{T}^{(2, \alpha)}+\left\langle w_{n-2}\right\rangle_{T}^{(2, \alpha)}\right)\left\langle w_{n-1}-w_{n-2}\right\rangle_{T}{ }^{(2, \alpha)} \\
\left|D_{x}\left\{g_{i j}\left(w_{n-1}\right)-g_{i j}\left(w_{n-2}\right)\right\}\right|_{x, T}^{(\alpha)} \\
\quad \leqq T \cdot B_{6}\left(T,\left\langle w_{n-1}\right\rangle_{T}{ }^{(2, \alpha)}+\left\langle w_{n-2}\right\rangle_{T}{ }^{(2, \alpha)}\right) \mid D_{x}{ }^{2}\left(w_{n-1}-w_{n-2}\right)_{T_{T}}^{(\alpha)} \\
\left|D_{x}\left\{g_{i j}\left(w_{n-1}\right)-g_{i j}\left(w_{n-2}\right)\right\}\right|_{i, T}^{(\alpha, 2)} \\
\leqq \\
\leqq B_{7}\left(T,\left\langle w_{n-1}\right\rangle_{T}{ }^{(2, \alpha)}+\left\langle w_{n-2}\right\rangle_{T}{ }^{(2, \alpha)}\right)\left\langle w_{n-1}-w_{n-2}\right\rangle_{T}{ }^{(2, \alpha)}
\end{array}\right.
$$

where $B_{5}, B_{6}$ and $B_{7}$ are monotorically increasing in each argument and $B_{5}, B_{7} \downarrow 0$ as $T \downarrow 0$. Hence we have

$$
\left\{\begin{array}{l}
\left|\hat{\rho}_{n-1}-\hat{\rho}_{n-2}\right| \leqq \bar{\rho}_{0} \cdot \exp \left[9 ( 1 + B _ { 1 } ) \left(\left\langle w_{n-1}\right\rangle_{T}{ }^{(2, \alpha)}+\left\langle w_{n-2}\right\rangle_{T}{ }^{(2, \alpha)}\right.\right. \\
\left.\left.\quad+\left\|v_{0}\right\|^{(2+\alpha)}\right) T\right] \cdot T\left\{B_{5}+9\left(1+B_{1}\right)\right\}\left\langle w_{n-1}-w_{n-2}\right\rangle_{T}{ }^{(2, \alpha)} \\
\left|D_{x}\left(\hat{\rho}_{n-1}-\hat{\rho}_{n-2}\right)\right| \leqq 3\left[\left|\rho_{0}^{\prime}\right|^{(0)}+\overline{\bar{\rho}}_{0}\left\{1+9\left(1+B_{1}+B_{3}\right)\left(\left\langle w_{n-1}\right\rangle_{T}{ }^{(2, \alpha)}\right.\right.\right. \\
\left.\left.\left.+\left\|v_{0}\right\|^{(2+\alpha)}\right)\right\}\right] T\left\{B_{5}+3\left(1+B_{1}\right)\right\} \cdot \exp \left[9 ( 1 + B _ { 1 } ) \left(\left\langle w_{n-1}\right\rangle_{T}{ }^{(2, \alpha)}\right.\right. \\
\left.\left.\quad+\left\langle w_{n-2}\right\rangle_{T}^{(2, \alpha)}+\left\|v_{0}\right\|^{(2+\alpha)}\right) T\right]\left\langle w_{n-1}-w_{n-2}\right\rangle_{T}{ }^{(2, \alpha)}, \\
\left|D_{t}\left(\hat{\rho}_{n-1}-\hat{\rho}_{n-2}\right)\right| \leqq \overline{\bar{\rho}}_{0}\left[270\left(1+B_{1}+B_{3}\right)\left(\left\langle w_{n-1}\right\rangle_{T}{ }^{(2, \alpha)}+\left\|v_{0}\right\|^{(2+\alpha)}\right)\right. \\
\left.\quad \times\left\{B_{5}\left(\left\langle w_{n-1}\right\rangle_{T}{ }^{(2, \alpha)}+\left\|v_{0}\right\|^{(2+\alpha)}\right)+1+B_{1}+B_{3}\right\}\right] \cdot \exp \left[9\left(1+B_{1}\right)\right. \\
\left.\quad \times\left(\left\langle w_{n-1}\right\rangle_{T}{ }^{(2, \alpha)}+\left\langle w_{n-2}\right\rangle_{T}{ }^{(2, \alpha)}+\left\|v_{0}\right\|^{(2+\alpha)}\right) T\right] \\
\quad \times\left\langle w_{n-1}-w_{n-2}\right\rangle_{T}^{(2, \alpha)}
\end{array}\right.
$$

where

$$
\begin{aligned}
& \hat{\rho}_{n-1}(x, t)=\rho_{0}(x) \cdot \exp \left[-\int_{0}^{t} g_{i j}\left(w_{n-1}\right) \partial_{j}\left(w_{n-1, i}+v_{0, i}\right) d \tau\right] \\
& \left\{\begin{array}{l}
\left|\frac{1}{\hat{\rho}_{n-1}}-\frac{1}{\hat{\rho}_{n-2}}\right| \leqq\left(\bar{\rho}_{0}\right)^{-2} \cdot \exp \left[9 ( 1 + B _ { 1 } ) \left(\left\langle w_{n-1}\right\rangle_{T}{ }^{(2, \alpha)}+\left\langle w_{n-2}\right\rangle_{T}{ }^{(2, \alpha)}\right.\right. \\
\left.\left.\quad+\left\|v_{0}\right\|^{(2+\alpha)}\right) T\right] \cdot\left|\hat{\rho}_{n-1}-\hat{\rho}_{n-2}\right|, \\
\left|\Delta_{x}{ }^{x_{0}}\left(\hat{\rho}_{n-1}^{-1}-\hat{\rho}_{n-2}^{-1}\right)\right| \leqq 2\left|x-x_{0}\right|^{\alpha}\left\{\mid \hat{\rho}_{n-1}^{-2} \hat{\rho}_{n-2}^{-2}\left[D_{x}\left(\hat{\rho}_{n-1}-\hat{\rho}_{n-2}\right) \cdot \hat{\rho}_{n-1}^{2}\right.\right. \\
\left.\left.\quad+D_{x} \hat{\rho}_{n-2}\left(\hat{\rho}_{n-1}^{2}-\hat{\rho}_{n-2}^{2}\right)\right]\left.\right|_{T}{ }^{(0)}+\left|\hat{\rho}_{n-1}^{-1}-\hat{\rho}_{n-2}^{-1}\right|_{T}{ }^{(0)}\right\}, \\
\left|\Delta_{t}^{t_{0}}\left(\hat{\rho}_{n-1}^{-1}-\hat{\rho}_{n-2}^{-1}\right)\right| \leqq 2\left(t-t_{0}\right)^{\alpha / 2}\left\{\left|D_{t}\left(\hat{\rho}_{n-1}^{-1}-\hat{\rho}_{n-2}^{-1}\right)\right|_{T}{ }^{(0)}\right. \\
\left.\quad+\left|\hat{\rho}_{n-1}^{-1}-\hat{\rho}_{n-2}^{-1}\right|_{T}{ }^{(0)}\right\} .
\end{array}\right.
\end{aligned}
$$




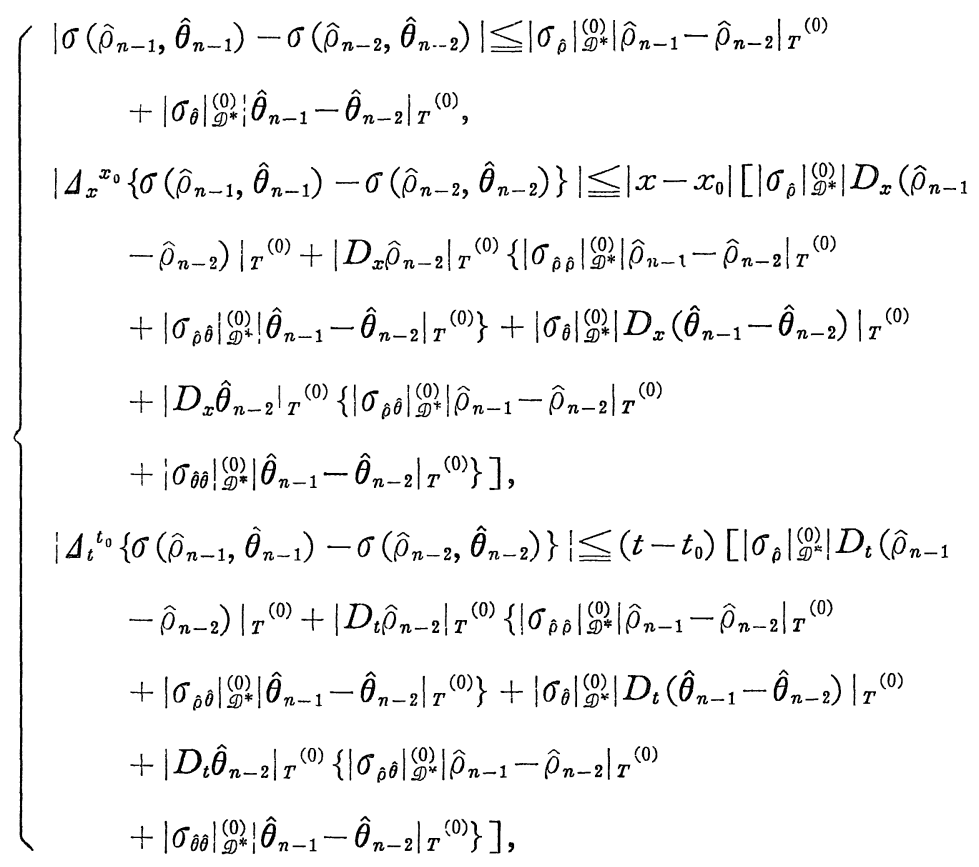

where

$$
\mathscr{D})^{*}=\mathscr{D}_{\rho, \hat{\theta}}^{*}, \quad|\sigma|_{\mathscr{D}^{+}}^{(0)}=\max _{\mathscr{D}^{*}}|\sigma|
$$

Since $\left(\hat{\theta}_{n-1}-\hat{\theta}_{n-2}\right)(x, 0)=0$, we have

$$
\left|\left(\hat{\theta}_{n-1}-\hat{\theta}_{n-2}\right)(x, t)\right| \leqq t\left|D_{t}\left(\hat{\theta}_{n-1}-\hat{\theta}_{n-2}\right)\right|_{T}{ }^{(0)} .
$$

Lemma 6. 2. If $g(x, t)$ be defined in $\bar{Q}_{T}, g_{x}$ exist,

$$
\left|\Delta_{t}^{t_{0}} g\right| \leqq A_{3}\left(t-t_{0}\right)^{\alpha}, \quad\left|\Delta_{x}^{x_{0}} D_{x} g\right| \leqq A_{4}\left|x-x_{0}\right|^{\beta}
$$

and $\Omega$ satisfy the cone condition, then

$$
\left|\Delta_{t}{ }^{t_{0}} D_{x} g\right| \leqq A_{5}\left(t-t_{0}\right)^{\alpha \beta /(1+\beta)} .
$$

Proof. See, e.g., [33].

From (6.3)-(6.7) and Lemma 6.2 follows that

$$
\left\{\begin{array}{l}
\mathfrak{U}_{i j}^{k m}\left(x, t, w_{n-1}\right)-\mathfrak{U}_{i j}^{k m}\left(x, t, w_{n-2}\right) \in H^{\alpha}\left(\bar{Q}_{T}\right), \\
\left\|\mathfrak{P}_{i j}^{k m n}\left(x, t, w_{n-1}\right)-\mathfrak{U}_{i j}^{k m}\left(x, t, w_{n-2}\right)\right\|_{T}{ }^{(\alpha)} \leqq b_{4}\left(T,\left\langle w_{n-1}\right\rangle_{T}{ }^{(2, \alpha)}\right. \\
\left.\quad+\left\langle w_{n-2}\right\rangle_{T}{ }^{(2, \alpha)}\right)\left\langle w_{n-1}-w_{n-2}\right\rangle_{T}{ }^{(2, \alpha)}
\end{array}\right.
$$


where $b_{4}$ is monotonically increasing in each argument and $b_{4} \downarrow 0$ as $T \downarrow 0$. Thus we have

(6. 9) $\quad\left\|\left\{\mathfrak{U}\left(x, t, w_{n-1} ; D_{x}\right)-\mathfrak{U}\left(x, t, w_{n-2} ; D_{x}\right)\right\} w_{n-1}\right\|_{T}{ }^{(\alpha)}$

$$
\begin{aligned}
& \leqq b_{5}\left(T,\left\langle w_{n-1}\right\rangle_{T}{ }^{(2, \alpha)}+\left\langle w_{n-2}\right\rangle_{T}{ }^{(2, \alpha)},\left|D_{x}{ }^{2} w_{n-1}\right|_{T}{ }^{(\alpha)}\right) \\
& \times\left\langle w_{n-1}-w_{n-2}\right\rangle_{T}{ }^{(2, \alpha)},
\end{aligned}
$$

where $b_{5}$ has the same property as $b_{4}$.

In the next place we have

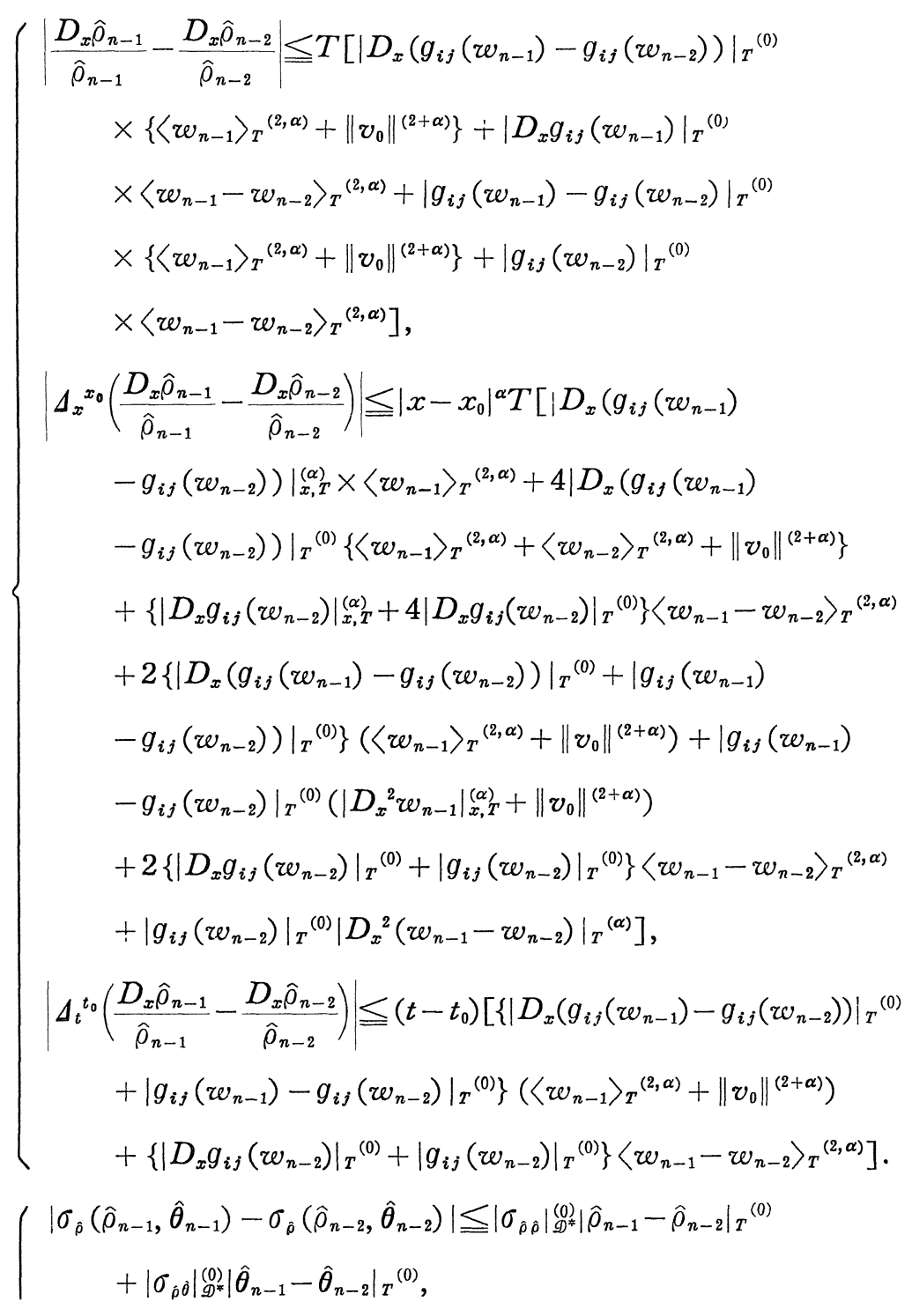


(6. 11)

$$
\begin{aligned}
& \left|\Delta_{x}^{x_{0}}\left\{\sigma_{\hat{\rho}}\left(\hat{\rho}_{n-1}, \hat{\theta}_{n-1}\right)-\sigma_{\hat{\rho}}\left(\hat{\rho}_{n-2}, \hat{\theta}_{n-2}\right)\right\}\right| \leqq\left|x-x_{0}\right|\left[\left.\left|\sigma_{\hat{\rho} \hat{\rho}}\right|\right|_{\mathscr{Q}^{*}} ^{(0)} \mid D_{x}\left(\hat{\rho}_{n-1}\right.\right. \\
& \left.-\hat{\rho}_{n-2}\right)\left.\right|_{T}{ }^{(0)}+\left|D_{x} \hat{\rho}_{n-2}\right| T_{T}{ }^{(0)}\left|\sigma_{\hat{\rho} \hat{\rho}}\right|_{\hat{\theta}, D^{*}}^{(L)}\left|\hat{\theta}_{n-1}-\hat{\theta}_{n-2}\right|_{T}{ }^{(0)} \\
& +\left|\sigma_{\hat{\rho} \hat{\theta}}\right|_{\mathscr{D}}^{(0)}\left|D_{x}\left(\hat{\theta}_{n-1}-\hat{\theta}_{n-2}\right)\right|_{T}{ }^{(0)}+\left|D_{x} \hat{\theta}_{n-2}\right|_{T}{ }^{(0)}\left|\sigma_{\hat{\rho} \hat{\theta}}\right|_{\hat{p}, \mathscr{D}^{*}}^{(L)} \\
& \left.\times\left|\hat{\rho}_{n-1}-\hat{\rho}_{n-2}\right|_{T}^{(0)}\right], \\
& \left|\Delta_{t}^{t_{0}}\left\{\sigma_{\hat{\rho}}\left(\hat{\rho}_{n-1}, \hat{\theta}_{n-1}\right)-\sigma_{\hat{\rho}}\left(\hat{\rho}_{n-2}, \hat{\theta}_{n-2}\right)\right\}\right| \leqq\left(t-t_{0}\right)\left[\left.\left|\sigma_{\hat{\rho} \hat{\rho}}\right|\right|_{\mathscr{D}^{*}} ^{(0)}\right. \\
& \times\left|D_{t}\left(\hat{\rho}_{n-1}-\hat{\rho}_{n-2}\right)\right|_{T}{ }^{(0)}+\left.\left|D_{t} \hat{\rho}_{n-2}\right| T^{(0)}\left|\sigma_{\hat{\rho} \hat{\rho}}\right|\right|_{\hat{\theta}, \mathscr{D}^{*}} ^{(L)}\left|\hat{\theta}_{n-1}-\hat{\theta}_{n-2}\right|_{T}{ }^{(0)} \\
& +\left|\sigma_{\hat{\rho} \hat{\theta}}\right|_{\mathscr{D}^{*}}^{(0)}\left|D_{t}\left(\hat{\theta}_{n-1}-\hat{\theta}_{n-2}\right)\right|_{T}{ }^{(0)}+\left|D_{t} \hat{\theta}_{n-2}\right|_{T}{ }^{(0)}\left|\sigma_{\hat{\rho} \hat{\theta}}\right|_{\hat{\rho}, \mathscr{D}^{*}}^{(L)} \\
& \left.\times\left|\hat{\rho}_{n-1}-\hat{\rho}_{n-2}\right|_{T}{ }^{(0)}\right],
\end{aligned}
$$

where

$$
\left\{\begin{array}{l}
|\sigma|_{\hat{\rho}, \mathscr{D}^{*}}^{(L)}=\max _{(\hat{\rho}, \hat{\theta}),\left(\hat{\rho}^{\prime}, \hat{\theta}\right) \in \mathscr{D}^{*}, \hat{\rho} \neq \hat{\rho}^{\prime}} \frac{\left|\sigma(\hat{\rho}, \hat{\theta})-\sigma\left(\hat{\rho}^{\prime}, \hat{\theta}\right)\right|}{\left|\hat{\rho}-\hat{\rho}^{\prime}\right|} \\
|\sigma|_{\hat{\theta}, \mathscr{D}^{*}}^{(L)}=\max _{(\hat{\rho}, \hat{\theta}),\left(\hat{\hat{\rho}}, \hat{\theta}^{\prime}\right) \in \mathscr{D}^{*}, \hat{\theta} \neq \hat{\theta}^{\prime}} \frac{\left|\sigma(\hat{\rho}, \hat{\theta})-\sigma\left(\hat{\rho}, \hat{\theta}^{\prime}\right)\right|}{\left|\hat{\theta}-\hat{\theta}^{\prime}\right|}
\end{array}\right.
$$

As for $\sigma_{\hat{\theta}^{\prime}}$ we can obtain the similar estimates for it to those of $\sigma_{\hat{p}}$.

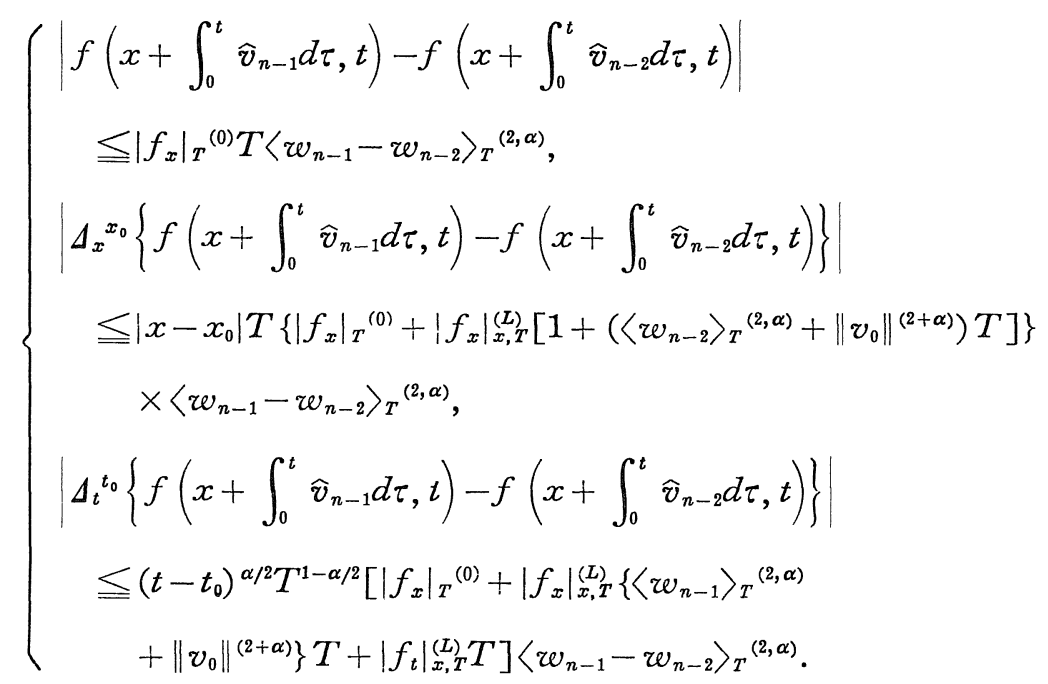

From (2.20), (5.36), (5.37) with $w_{n-1}$ and $w_{n-2},(6.3),(6.7)$, (6.10)-(6.12) and Lemma 6.2 it follows that

(6. 13) $\quad\left\|\mathfrak{B}\left(x, t, w_{n-1}\right)-\mathfrak{B}\left(x, t, w_{n-2}\right)\right\|_{T}{ }^{(\alpha)}$

$$
\leqq b_{6}\left(T,\left\langle w_{n-1}\right\rangle_{T}{ }^{(2, \alpha)}+\left\langle w_{n-2}\right\rangle_{T}{ }^{(2, \alpha)},\left|D_{x}^{2} w_{n-1}\right|_{T}{ }^{(\alpha)}\right.
$$




$$
\begin{aligned}
& \left.+\left|D_{x}^{2} w_{n-2}\right|_{T}^{(\alpha)}\right)\left\langle w_{n-1}-w_{n-2}\right\rangle_{T}{ }^{(2, \alpha)}+b_{7}\left(T,\left\langle w_{n-1}\right\rangle_{T}{ }^{(2, a)}\right. \\
& \left.+\left\langle w_{n-2}\right\rangle_{T}{ }^{(2, \alpha)}\right)\left|D_{x}{ }^{2}\left(w_{n-1}-w_{n-2}\right)\right|_{T}{ }^{(\alpha)}
\end{aligned}
$$

where $b_{6}$ and $b_{7}$ have the same property as $b_{4}$.

Hence a solution of (6.2) is given by

$$
\begin{aligned}
\left(w_{n}-w_{n-1}\right) & (x, t)=\int_{0}^{t} d \tau \int_{\Omega} G\left(x, t ; \xi, \tau ; w_{n-1}\right) \\
& \times\left[\left\{\mathfrak{U}\left(\xi, \tau, w_{n-1} ; D_{x}\right)-\mathfrak{H}\left(\xi, \tau, w_{n-2} ; D_{x}\right)\right\} w_{n-1}\right. \\
& \left.+\mathfrak{B}\left(\xi, \tau, w_{n-1}\right)-\mathfrak{B}\left(\xi, \tau, w_{n-2}\right)\right] d \xi
\end{aligned}
$$

From Lemma 5.1 follows that (cf. $(5,41)$ )

$$
\left\{\begin{array}{l}
\left\langle w_{n}-w_{n-1}\right\rangle_{T}{ }^{(2, \alpha)} \\
\leqq \\
\quad C_{51}\left(T,\left\langle w_{n-1}\right\rangle_{T}{ }^{(2, \alpha)}\right)\left(b_{5}+b_{6}\right)\left\langle w_{n-1}-w_{n-2}\right\rangle_{T}{ }^{(2, \alpha)} \\
\quad+C_{51} b_{7}\left|D_{x}{ }^{2}\left(w_{n-1}-w_{n-2}\right)\right|_{T}{ }^{(\alpha)} \\
\left|D_{x}{ }^{2}\left(w_{n}-w_{n-1}\right)\right|_{T}{ }^{(\alpha)} \leqq C_{52}\left(b_{5}+b_{6}\right)\left\langle w_{n-1}-w_{n-2}\right\rangle_{T}{ }^{(2, \alpha)} \\
\quad+C_{52} b_{7}\left|D_{x}{ }^{2}\left(w_{n-1}-w_{n-2}\right)\right|_{T}{ }^{(\alpha)}
\end{array}\right.
$$

Now we denote $\langle w\rangle_{T}{ }^{(2, \alpha)}+\left|D_{x}{ }^{2} w\right|_{T}{ }^{(\alpha)}$ by $\left\langle\langle w\rangle_{T}{ }^{(2, \alpha)}\right.$. Then from (6.15) we derive

$$
\begin{aligned}
\left\langle\left\langle w_{n}-w_{n-1}\right\rangle_{T}{ }^{(2, \alpha)} \leqq\right. & C_{53}\left(T,\left\langle\left\langle w_{n-1}\right\rangle_{T}{ }^{(2, \alpha)}+\left\langle\left\langle w_{n-2}\right\rangle_{T}{ }^{(2, \alpha)}\right)\right.\right. \\
& \times\left\langle\left\langle w_{n-1}-w_{n-2}\right\rangle_{T}{ }^{(2, \alpha)},\right.
\end{aligned}
$$

where $C_{53}$ is monotonically increasing in each argument and $C_{53} \downarrow 0$ as $T \downarrow 0$. By induction we have

$$
\left\langle\left\langle w_{n}-w_{n-1}\right\rangle_{T}{ }^{(2, \alpha)} \leqq C_{53}^{n-1}\left\langle\left\langle w_{1}-w_{0}\right\rangle_{T}{ }^{(2, \alpha)} .\right.\right.
$$

The property of $C_{53}$ implies that for some $T^{\prime} \in(0, T]$

$$
C_{53}\left(T^{\prime}, 2 M_{1}+2 M_{2}\right)<1 \text {. }
$$

Moreover since

$$
\left.\left\langle w_{1}-w_{0}\right\rangle\right\rangle_{T^{\prime}}^{(2, \alpha)}=\left\langle\left\langle w_{1}\right\rangle_{T^{\prime}}^{(2, \alpha)} \leqq 2\left(C_{51}\left(T^{\prime}\right)+C_{52}\left(T^{\prime}\right)\right) b_{2}\left(T^{\prime}\right)<+\infty,\right.
$$

we have

$$
\sum_{n=1}^{\infty} C_{53}^{n-1}\left\langle\left\langle w_{1}-w_{0}\right\rangle_{T^{\prime}}^{(2, \alpha)}<+\infty\right.
$$


That is to say, $\left\{w_{n}\right\}$ converge to an element $w$ of $H^{2+\alpha}\left(\bar{Q}_{T^{\prime}}\right)$ as $n \rightarrow \infty$. As is known the expression of $\rho_{n},\left\{\rho_{n}\right\}$ converge to an element $\rho$ of $B^{1+\alpha}\left(\bar{Q}_{T^{\prime}}\right)$ as $n \rightarrow \infty . \quad \mathfrak{U}\left(x, t, w_{n} ; D_{x}\right), \mathfrak{B}\left(x, t, \mathfrak{w}_{n}\right)$ and $G(x, t ; \xi, \tau$; $\left.w_{n}\right)$ also converge to $\mathfrak{H}\left(x, t, w ; D_{x}\right), \mathfrak{B}(x, t, w)$ and $G(x, t ; \xi, \tau ; w)$, respectively. Consequently a solution of $(2.15)-(2.16)$ is given by

$$
w(x, t)=\int_{0}^{t} d \tau \int_{\Omega} G(x, t ; \xi, \tau ; w) \mathfrak{B}(\xi, \tau, w) d \xi
$$

Remark. For w', Lemma 5.1 also holds with $\phi=\mathfrak{B}$.

\section{$\S$ 7. The Proof of Uniqueness}

Now let us direct ourselves towards the problem of uniqueness concerning (2.15). We assume that there exist two solutions (w, $\hat{\rho}$ ) and $\left(w^{*}, \hat{\rho}^{*}\right)$ of $(2.15)$ in $H^{2+\alpha}\left(\bar{Q}_{T}\right) \times B^{1+\alpha}\left(\bar{Q}_{T}\right)$ satisfying one and the same initial-boundary condition (2.16). The difference $w-w^{*}$ satisfies (6. 2) as $w_{n}$ and $w_{n-1}$ are replaced by $w$ and $w^{* *}$ respectively. Then $w-w^{*}$ can be uniquely expressed in the form (6.14) as $w_{n}$ and $w_{n-1}$ are replaced by $w$ and $w^{*}$ respectively, i.e.,

$$
\begin{aligned}
& \left(w-w^{*}\right)(x, t)=\int_{0}^{t} d \tau \int_{\Omega} G(x, t ; \xi, \tau ; w) \\
& \quad \times\left\{\left[\mathfrak{I}\left(\xi, \tau, w ; D_{x}\right)-\mathfrak{U}\left(\xi, \tau, w^{*} ; D_{x}\right)\right] w^{*}\right. \\
& \left.\quad+\mathfrak{B}(\xi, \tau, w)-\mathfrak{B}\left(\xi, \tau, w^{*}\right)\right\} d \xi .
\end{aligned}
$$

As for $w-w^{*}$, in a way analogous to that used in the preceding section for $w_{n}-w_{n-1}$, we have for some constant $C_{54}$ having the same property as $C_{53}$

$(7.2) \quad\left\langle w w-w^{*}\right\rangle_{T}^{(2, \alpha)} \leqq C_{54}\left(T,\left\langle\langle w\rangle_{T}^{(2, a)}+\left\langle\left\langle\mathcal{w}^{*}\right\rangle_{T}{ }^{(2, \alpha)}\right)\left\langle\psi w-w^{*}\right\rangle_{T}^{(2, \alpha)}\right.\right.$.

Hence there exists $T_{0} \in\left(0, T^{\prime}\right]$ such that

$$
C_{54}\left(T_{0}\right)<1 .
$$

From these we derive $w(x, t)=w^{*}(x, t)$ for $0 \leqq t \leqq T_{0} \leqq T$. Hereafter, it remains only to make a finite number of repetitions of the same procedure. The uniqueness of $\rho$ follows from that of $r$. Thus Theorem 2 is proved. 


\section{§8. The Proof of Theorem $\mathbb{1}$}

By Theorem 2 and (2.15) there exists a unique solution $(\widehat{v}, \hat{\theta}, \hat{\rho}) \in$ $H^{2+\alpha}\left(\bar{Q}_{T^{\prime}}\right) \times H^{2+\alpha}\left(\bar{Q}_{T^{\prime}}\right) \times B^{1+\alpha}\left(\bar{Q}_{T^{\prime}}\right)$. Now according to the notations in $\S 2$, we respectively define $x$ and $(v, \theta, \rho)$ by $(2.5)$ and

$$
\left\{\begin{array}{l}
v(x, t)=\hat{v}\left(x_{0}(x, t), t_{0}=t\right), \\
\theta(x, t)=\hat{\theta}\left(x_{0}(x, t), t_{0}=t\right), \\
\rho(x, t)=\hat{\rho}\left(x_{0}(x, t), t_{0}=t\right) .
\end{array}\right.
$$

Since $(v, \theta, \rho)$ certainly satisfies (1.1), (1.2), (1.3)' and (1.5), it is sufficient to prove $(v, \theta, \rho) \in H^{2+\alpha}\left(\bar{Q}_{T^{\prime}}\right) \times H^{2+\alpha}\left(\bar{Q}_{T^{\prime}}\right) \times B^{1+\alpha}\left(\bar{Q}_{T^{\prime}}\right)$ and the uniqueness. Here we do those of $v$ only.

Lemma 8. 1. If $\widehat{v} \in H^{2+\alpha}\left(\bar{Q}_{T}\right)$, then $v \in H^{2+\alpha}\left(\bar{Q}_{T}\right)$.

Proof. We prove that $\left|D_{x}{ }^{2} v\right|_{t, T}^{(\alpha / 2)}<+\infty$ only. Other estimates can be derived analogously.

$$
\begin{aligned}
& \left|\Delta_{t}{ }^{t^{\prime}} D_{x}{ }^{2} v\right| \leqq\left|\Delta_{t}{ }^{t^{\prime}} D_{x} g_{i j}\right|_{T}{ }^{(0)}\left|D_{x_{0}} \widehat{v}\right|_{T}{ }^{(0)}+\left|D_{x} g_{i j}\right|_{T}{ }^{(0)}\left|\Delta_{t}{ }^{t^{\prime}} D_{x_{0}} \widehat{v}\left(x_{0}(x, t), t_{0}=t\right)\right|_{T}{ }^{(0)} \\
& +\left|\Delta_{t}{ }^{t^{\prime}}\left(D_{x} x_{0}\right)^{2}\right|_{T}{ }^{(0)}+\left|\left(D_{x} x_{0}\right)^{2}\right|_{T}{ }^{(0)}\left|\Delta_{t}{ }^{\prime} D_{x_{0}}^{2} \widehat{v}\right|_{T}{ }^{(0)} \\
& \leqq\left|t-t^{\prime}\right|^{\alpha / 2}\left[\left|D_{x_{0}} \widehat{v}\right|_{T}{ }^{(0)} B_{3} T^{1-\alpha / 2}\left\{\langle\widehat{v}\rangle_{T}{ }^{(2, \alpha)}+\left\|v_{0}\right\|^{(2+\alpha)}\right\}\right. \\
& +9 B_{3}\left\{9\left(1+B_{1}\right)|\widehat{v}|_{T}{ }^{(0)}\left|D_{x_{0}}^{2} \widehat{v}\right|_{T}{ }^{(0)}+2\left|D_{x_{0}} \widehat{v}\right|_{T}{ }^{(0)}+\left|D_{x_{0}} \widehat{v}\right|_{t, T}^{(\alpha / 2)}\right\} \\
& +162 B_{3}\left(1+B_{1}\right) T^{1-\alpha / 2}\left|D_{x_{0}}^{2} \widehat{v}\right|_{T}{ }^{(0)}+81\left(1+B_{1}\right)^{2}\left\{T^{\alpha / 2} 9^{\alpha}\left(1+B_{1}\right)^{\alpha}\right. \\
& \left.\left.\times\left(|\widehat{v}|_{T}^{(0)}\right)^{\alpha}\left|D_{x_{0}}^{2} \widehat{v}\right|_{x, T}^{(\alpha)}+\left|D_{x_{0}}^{2} \widehat{v}\right|_{t, T}^{(\alpha / 2)}\right\}\right],
\end{aligned}
$$

hence $\left|D_{x}^{2} v\right|_{t, T}^{(\alpha / 2)}<+\infty$.

Q.E.D.

Lemma 8. 2. The mapping $v=F \widehat{v}$ from $H^{2+\alpha}\left(\bar{Q}_{T}\right)$ onto itself is ond to one.

Proof. Suppose that $v=v^{*}$, i.e., $F \widehat{v}=F \widehat{v}^{*}$. Then from (2.4) we derive

$$
\int \frac{d}{d \tau} x\left(x_{0}, \tau ; \widehat{v}\right)=\widehat{v}\left(x_{0}, \tau\right) \equiv v\left(x\left(x_{0}, \tau ; \widehat{v}\right), \tau\right),
$$




$$
\begin{array}{r}
x\left(x_{0}, 0 ; \widehat{v}\right)=x_{0} ; \\
\frac{d}{d \tau} x\left(x_{0}, \tau ; \widehat{v}^{*}\right)=\widehat{v}^{*}\left(x_{0}, \tau\right) \equiv v^{*}\left(x\left(x_{0}, \tau ; \widehat{v}^{*}\right), \tau\right), \\
x\left(x_{0}, 0 ; \widehat{v}^{*}\right)=x_{0} .
\end{array}
$$

Therefore we have

$$
\left\{\begin{array}{l}
\frac{d}{d \tau}\left(x\left(x_{0}, \tau ; \widehat{v}\right)-x\left(x_{0}, \tau ; \widehat{v}^{*}\right)\right) \\
=v\left(x\left(x_{0}, \tau ; \widehat{v}\right), \tau\right)-v\left(x\left(x_{0}, \tau ; \widehat{v}^{*}\right), \tau\right), \\
x\left(x_{0}, 0 ; \widehat{v}\right)-x\left(x_{0}, 0 ; \widehat{v}^{*}\right)=0,
\end{array}\right.
$$

hence

$$
\left\{\begin{array}{l}
\frac{1}{2} \frac{d}{d \tau}\left(x\left(x_{0}, \tau ; \widehat{v}\right)-x\left(x_{0}, \tau ; \widehat{v}^{*}\right)\right)^{2} \\
\leqq\left|v_{x}\right|_{T}^{(0)}\left(x\left(x_{0}, \tau ; \widehat{v}\right)-x\left(x_{0}, \tau ; \widehat{v}^{*}\right)\right)^{2}, \\
x\left(x_{0}, 0 ; \widehat{v}\right)-x\left(x_{0}, 0 ; \widehat{v}^{*}\right)=0 .
\end{array}\right.
$$

From (8.4) it follows that $x\left(x_{0}, \tau ; \widehat{v}\right)=x\left(x_{0}, \tau ; \widehat{v}^{*}\right)$, hence $\widehat{v}\left(x_{0}, t\right)$ $=\widehat{v}^{*}\left(x_{0}, t\right)$.

Q.E.D.

By Lemmas 8.1 and 8.2 the proof of Theorem 1 is now completed.

\section{References}

[1] Agmon, S., Douglis, A. and Nirenberg, L., Estimates near the boundary for solutions of elliptic partial differential equations satisfying general boundary conditions, I, Comm. Pure Appl. Math., 12 (1959), 623-727; II, Ibid., 17 (1964), 35-92.

[2] Arima, R., On general boundary value problem for parabolic equations. J, Math. Kyoto Univ., 4 (1964), 207-243.

[3] Bergmann, S., Integral operators in the theory of linear partial differential equa. tions, Springer, 1961.

[4] Browder, F. E., A priori estimates for solutions of elliptic boundary-value problems, I, Proc. Koninklijke Nederl. Akad. van Wetenshappen, ser. A, 63 (1960), 145-159: II, Ibid., 63 (1960), 160-169; III, Ibid., 64 (1961), 404-410.

[5] Browder, F. E., Estimates and existence theorems for elliptic boundary value problem, Proc. Nat. Acad. Sci. USA, 45 (1959), 365-372.

[6] Coddington, E. and Levinson, N., Theory of ordinary differential equations, McGraw-Hill, 1955.

[7] Courant, R. and Hilbert, D., Methods of mathematical physics, IJ, Interscience, 1962.

[8] Douglis, A. and Nirenberg, L., Interior estimates for elliptic systems of partial differential equations, Comm. Pure Appl. Math., 8 (1955), 503-538.

[9] Эидельман, С. Д., О фундаментальных решениях параболических систем, Mam. C6., 38 (1956), 51-92; II, Ibid., 53 (1961) 73-136. 
[10] Эидельман, С. Д., Об одном классе параболических систем, Докл. АН СССР, 133 (1960), 40-43.

[11] Эидельман, С. Д., О краевых задачах для параболических систем в полупространстве, Ibid., 142 (1962), 812-814.

[12] Эидельман, С. Д., К теории общих граничных задач для параболических систем, Ibid., 149 (1963), 792-795.

[13] Эидельман, С. Д., Параболические системы, Наука, 1964.

[14] Эидельман, С. Д. и Ивасишен, С. Д., Исследование матрицы Грина однородной параболической граничной задачи, Труды Москов. Мат. Об., 23 (1970), 179-234.

[15] Эидельман, С. Д. и Липко, Б. Я., О краевых задачах для параболических систем в областях общего вида, Докл. АН СССР, 150 (1963), 58-61.

[16] Friedman, A., Interior estimates for parabolic systems of partial differential equations, J. Math. Mech., 7 (1958), 393-417.

[17] Friedman, A., Boundary estimates for second order parabolic equations and their applications, Ibid., 7 (1958), 771-791.

[18] Friedman, A., Generalized functions and partial differential equations, PrenticeHall, 1963.

[19] Friedman, A., Partial differential equations of parabolic type, Prentice-Hall, 1964.

[20] Гельфанд, И. М. и Шилов, Г. Е., Обобщенные фбункиий, III, Москва, 1958.

[21] Гюнтер, Н. М., Теория потенииала и её при.менение $\kappa$ основным задачам математической бјизики, Гостехизат, 1953.

[22] Ивасишен, С. Д., Матрица Грина неоднородной параболической граничной

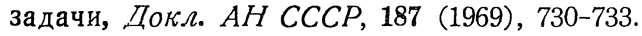

[23] Ивасишен, С. Д. и Эидельман, С. Д., Оценки матрицы Грина однородной параболической граничной задачи, Ibid., 172 (1967), 1262-1265.

[24] Itaya, N., The existence and uniqueness of the solution of the equations describing compressible viscous fluid flow, Proc. Japan Acad., 46 (1970), 379-382.

[25] Itaya, N., On the Cauchy problem for the system of fundamental equations describing the movement of compressible viscous fluid, Ködai Math. Sem. Rep., 23 (1971), 60-120.

[26] Itaya, N., A survey on the generalized Burgers' equation with a pressure model term, J. Math. Kyoto Univ., 16 (1976), 1-18.

[27] Itaya, N., On the fundamental system of equations for compressible viscous fluid, Sugaku, 28 (1976), 25-40 (Japanese).

[28] Itaya, N., On the initial value problem of the motion of compressible viscous fluid, especially on the problem of uniqueness, to appear in J. Math. Kyoto Univ.

[29] Красовский, Ю. П., Исследование потенциалов связанных с краевыми задачами для эллиптических уравнений, Нзв. АН, сер. матем., 31 (1967), 587-640.

[30] Красовский, Ю. П., Выделение особенности у функций Грина, Ibid., 31 (1967), 977-1010.

[31] Красовский, Ю. П., Свойства функций Грина и обобщения еллиптических граничных задач, Ibid., 33 (1969), 109-137.

[32] Ладыженская, О. А., Математические вопросы динамики взякой несжимаемой жидкости, Москва, 1970.

[33] Ладыженская, О. А., Солонников, В. А., и Уральцева, Н. Н., Линейные $u$ квазилинейные уравнения параболического типа, Москва, 1967.

[34] Lamb, H., Hydrodynamics, Cambridge, 1932.

[35] Lichtenstein, L., Grundlagen der Hydromechanik, Springer, 1929.

[36] Lions, J. L., Quelque méthodes de resolution des problème aux limites non linéaires, Gauthier-Villairs, 1969.

[37] Лопатинский, Я. Б., Фундаментальная система решений эллиптической системы линейных дифференциальных уравнений, Укр. Мат. Журн., 3 (1951), 3-38. 
[38] Лопатинский, Я. Б., Фундаментальных решения системы дифференциальных уравнений эллиптического типа, Ibid., 3 (1951), 290-316.

[39] Лопатинский, Я. Б., Об одном способе приведения граничных задач для системы дифференциальных уравнений эллиптического типа к регулярным интегральным уравнениям, Ibid., 5 (1953), 123-151.

[40] Meyer, R. E., Introduction to mathematiccil fuid dynamics, Wiley-Interscience, 1971.

[41] Михайлов, В. П., Решение смешаной задачи для параболической системы методом потенциалов, Док. АН СССР, 132 (1960), 291-294.

[42] Miranda, C., Equazioni alle derivate parziali di tipo elliptico, Springer, 1955.

[43] Nash, J., Parabolic equations, Proc. Nat. Acad. Sci. USA, 43 (1957), 754-758.

[44] Nash, J., Le problème de Cauchy pour les équations différentielles d'un fluide général, Bull. Soc. Math. France, 90 (1962), 487-497.

[45] Петровский, И. Г., Лекизии об уравнениях с иаспныльи произоднььиц, Гос. Изд. Москва, 1953.

[46] Pogorzelski, W., Étude de la solution fondamentale de l'équation parabolique, Ricerche Mat., 5 (1956), 25-57.

[47] Pogorzelski, W., Étude de la matrice des solutions fondamentales du systéme parabolique d'équations aux dérivées partielles, Ibid., 7 (1958), 153-185.

[48] Pogorzelski, W., Propriétés des solutions du systéme parabolique d'équations aux dérivées partielles, Mat. Scand., 6 (1958), 237-262.

[49] Schechter, M., General boundary value problems for elliptic partial differential equations, Comm. Pure Appl. Math., 12 (1959), 457-486.

[50] Serrin, J., On the uniqueness of compressible fluid motions, Arch. Rational Mech. Anal., 3 (1959), 271-288.

[51] Serrin, J., Mathematical principles of classical fluid mechanics, Handbuch der Physik, 8, Springer, 1959.

[52] Соломяк, М. З., Об условии Я. Б. Лопатинского разрешимости краевых задач, Вестник Ленинград. Унив. Сер. Мат. Мех. Физ., 1 (1965), 143-144.

[53] Солонников, В. А., Об общих краевых задачах для систем, эллиптических в смысле А. Даглиса-Л. Ниренберга, I, Нзв. АН, Сер. Мапеж., 28 (1964), 665706; II, Труды Мат. Ннст. Стеклов., 92 (1966), 233-296.

[54] Солонников, В. А., О краевых задачах для линейных параболических систем дифференциальных уравнений общего вида, Труды Мат. Инст. Стеклов., 83 (1965), 3-162.

[55] Truesdell, C., The mechanical foundations of elasticity and fluid dynamics, J. Rational Mech. Anal., 1 (1952), 125-300. 
